\title{
3 Research Square

\section{Downregulation of m6A reader YTHDC2 promotes the proliferation and migration of lung cancer and cigarette smoke-exposed BEAS-2B cells}

Jin Wang

Soochow University

Lirong Tan

Soochow University

Beibei Jia

Soochow University

Xiaofan Yu

Soochow University

Ruixin Yao

Soochow University

Nan Ouyang

Soochow University

Jian Tong

Soochow University

Tao Chen

Soochow University

Rui Chen

Second Affiliated Hospital of Soochow University

Jianxiang Li ( $\nabla$ aljxcr@suda.edu.cn )

Soochow University https://orcid.org/0000-0001-6674-7703

\section{Research}

Keywords: cigarette smoke, lung cancer, YTHDC2, proliferation, migration, bioinformatics

Posted Date: December 11th, 2020

DOl: https://doi.org/10.21203/rs.3.rs-125328/v1

License: (c) (1) This work is licensed under a Creative Commons Attribution 4.0 International License.

Read Full License 


\section{Abstract}

Background: Lung cancer is one of the most common carcinomas worldwide. Cigarette smoking is considered as the leading cause of lung cancer. The aberrant expression of several YTH family proteins has been reported to be closely associated with several cancers. This study aims to evaluate the expression profile and function of YTHDC2, an RNA m6 A methylation reader protein, by in vitro and animal experiments and bioinformatics analysis.

Methods: The expression of YTHDC2 gene and protein was analyzed by the GEPIA online tool and Gene Expression Omnibus (GEO) database, as well as immunohistochemistry of tissue arrays. The YTHDC2 expression in cigarette smoke-exposed cells was examined by RT-qPCR and western blot assays. The TCGA datasets and proteomic analysis of YTHDC2 knockdown cells were used to predict the clinical significance and biological function of YTHDC2. Functional assays, including wound healing, transwell migration, cell cycle and cell proliferation, were used to determine the biological role of YTHDC2 in lung cancer. The xenograft animal model further validated the tumor suppressor effect of YTHDC2 in vivo. The copy number variation of YTHDC2 was analyzed by TCGA datasets and TaqMan copy number assay.

Results: YTHDC2 was significantly low-expressed in lung cancer and cigarette smoke-exposed cells. Notably, decreased YTHDC2 was found highly associated with smoking history, pathological stage, invasion depth, and lymph node metastasis, as well as poor outcomes. The YTHDC2 related genes and proteins were enriched in several cancer-related pathways, and several tumor suppressor genes were found positively correlated with YTHDC2. The in vivo and in vitro study revealed that overexpression of YTHDC2 inhibited the proliferation and migration ability of lung cancer cells, as well as the tumor growth in nude mice. Besides, a significant positive correlation between the copy number and expression level of YTHDC2 was found in lung cancer.

Conclusions: Our results indicated that the smoking-related down-regulation of YTHDC2 was associated with enhanced proliferation and migration of lung cancer cells and appeared to be regulated by DNA copy number variation. Importantly, YTHDC2 may serve as a tumor suppressor gene and a favorable prognostic indicator in lung cancer patients.

\section{Full Text}

This preprint is available for download as a PDF.

\section{Figures}



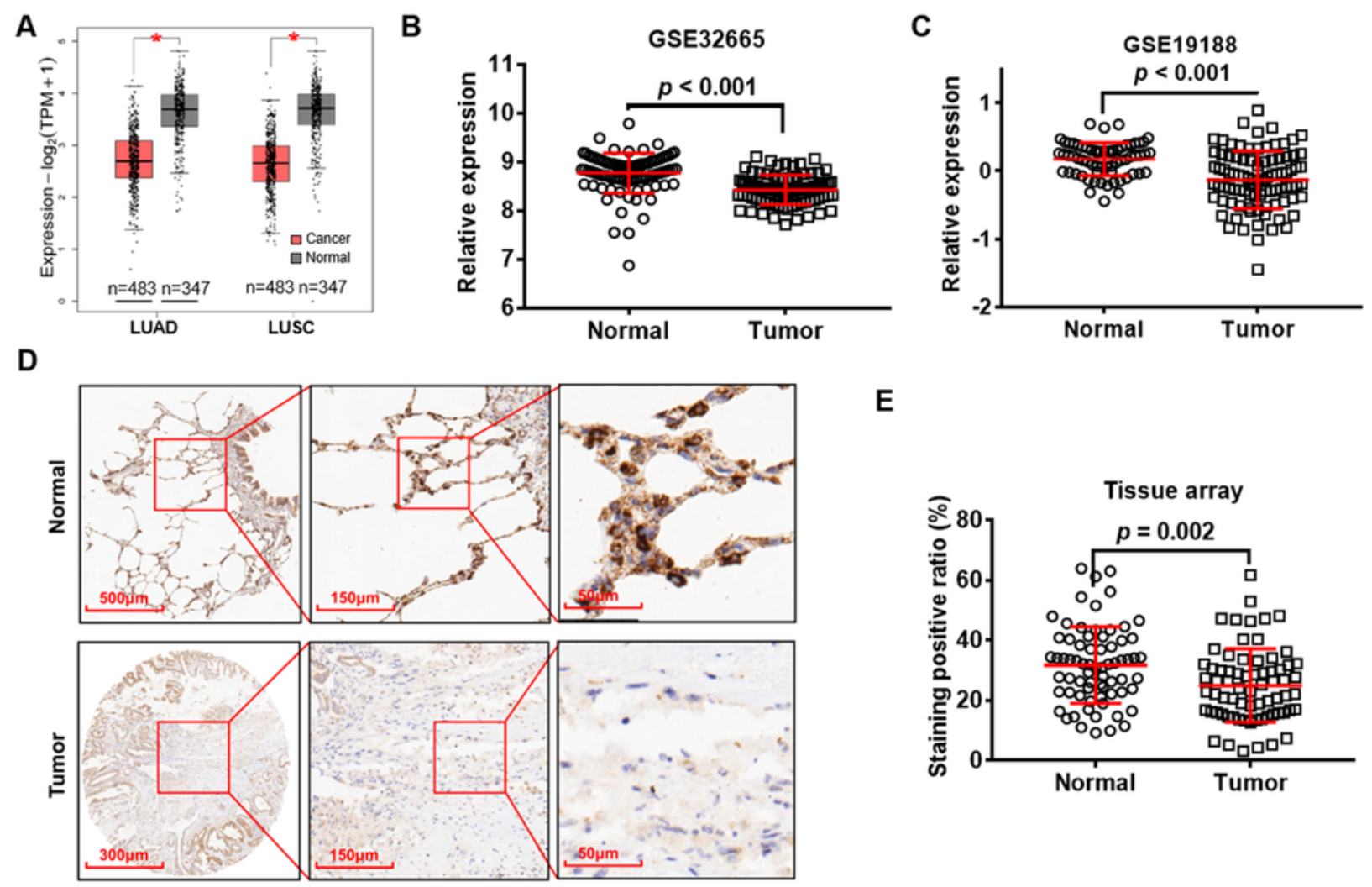

E
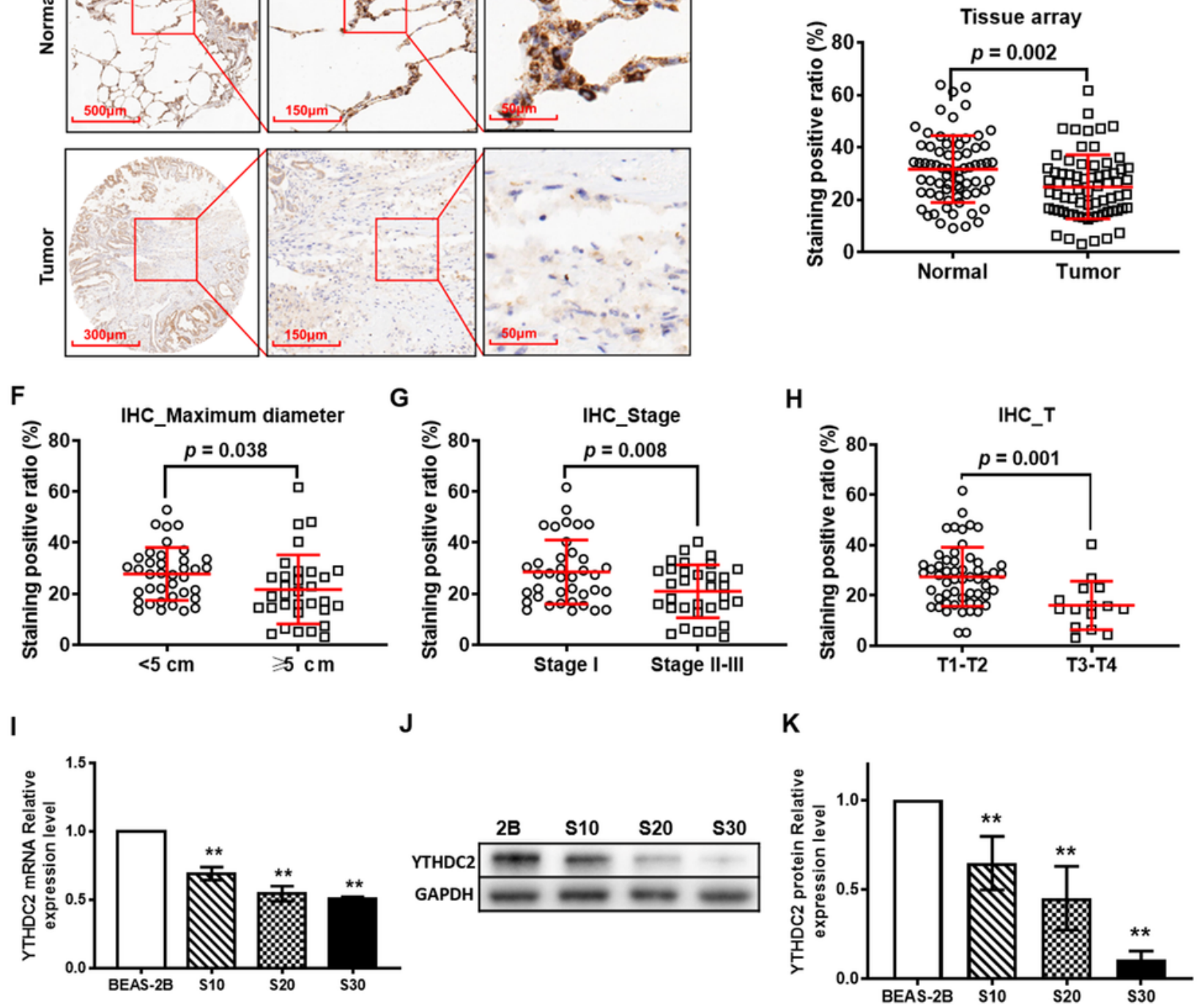

\section{Figure 1}

The YTHDC2 gene and protein were down-regulated in lung cancer patients from the TCGA, GEO and HPA databases, as well as in cigarette smoke-exposed cells. (A) Differential analysis of YTHDC2 mRNA expression in lung cancer tissues based on the GEPIA tool. ${ }^{*} p<0.05$ versus normal tissues. Differential analysis of YTHDC2 mRNA expression in lung cancer tissues in GSE32665 (B) and GSE19188 (C). (D) The representative IHC images showed that the YTHDC2 staining was found in the cytoplasm of the cells 
in lung cancer or normal lung tissues. High expression of YTHDC2 could be found in adjacent normal tissues, while its expression was decreased in most lung cancer tissues. (E) Differential analysis of YTHDC2 staining positive ratio quantitated by IHC profiler in lung cancer tissue arrays. (F) The relative mRNA expression level of YTHDC2 in cigarette smoke-exposed cells (S10, S20 and S30) and normal BEAS-2B cells. (G) Western blot analysis and quantitative results of YTHDC2 protein expression in cigarette smoke-exposed cells (S10, S20 and S30) and normal BEAS-2B cells. S10, S20 and S30 represent BEAS-2B cells exposed to cigarette smoke for 10,20 and 30 passages separately. ${ }^{*} p<0.01$ versus normal BEAS-2B cells. LUAD: lung adenocarcinoma; LUSC: Squamous cell carcinoma; IHC: Immunohistochemistry. 

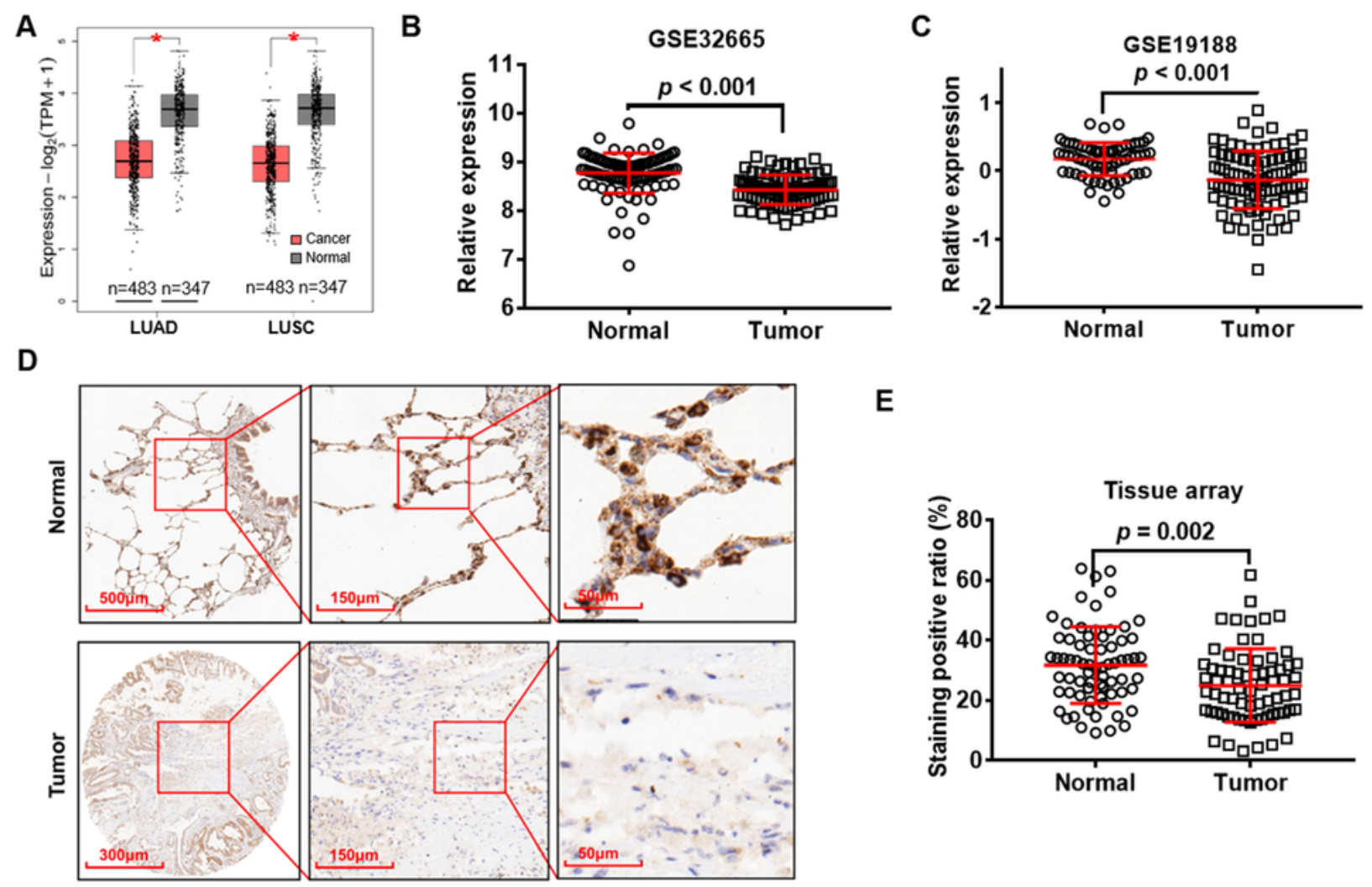

E
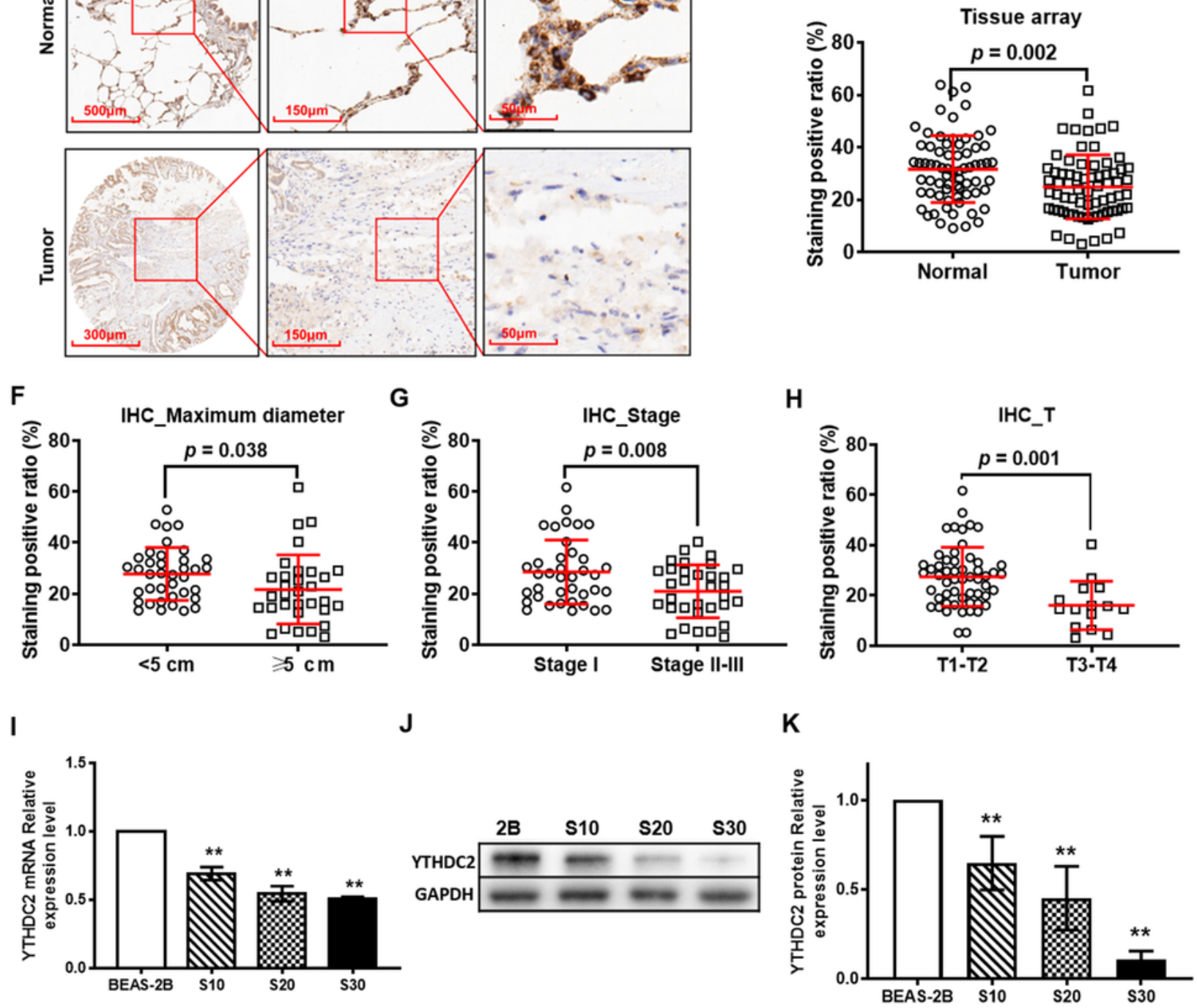

\section{Figure 1}

The YTHDC2 gene and protein were down-regulated in lung cancer patients from the TCGA, GEO and HPA databases, as well as in cigarette smoke-exposed cells. (A) Differential analysis of YTHDC2 mRNA expression in lung cancer tissues based on the GEPIA tool. ${ }^{*} p<0.05$ versus normal tissues. Differential analysis of YTHDC2 mRNA expression in lung cancer tissues in GSE32665 (B) and GSE19188 (C). (D) The representative IHC images showed that the YTHDC2 staining was found in the cytoplasm of the cells 
in lung cancer or normal lung tissues. High expression of YTHDC2 could be found in adjacent normal tissues, while its expression was decreased in most lung cancer tissues. (E) Differential analysis of YTHDC2 staining positive ratio quantitated by IHC profiler in lung cancer tissue arrays. (F) The relative mRNA expression level of YTHDC2 in cigarette smoke-exposed cells (S10, S20 and S30) and normal BEAS-2B cells. (G) Western blot analysis and quantitative results of YTHDC2 protein expression in cigarette smoke-exposed cells (S10, S20 and S30) and normal BEAS-2B cells. S10, S20 and S30 represent BEAS-2B cells exposed to cigarette smoke for 10,20 and 30 passages separately. ${ }^{*} p<0.01$ versus normal BEAS-2B cells. LUAD: lung adenocarcinoma; LUSC: Squamous cell carcinoma; IHC: Immunohistochemistry. 

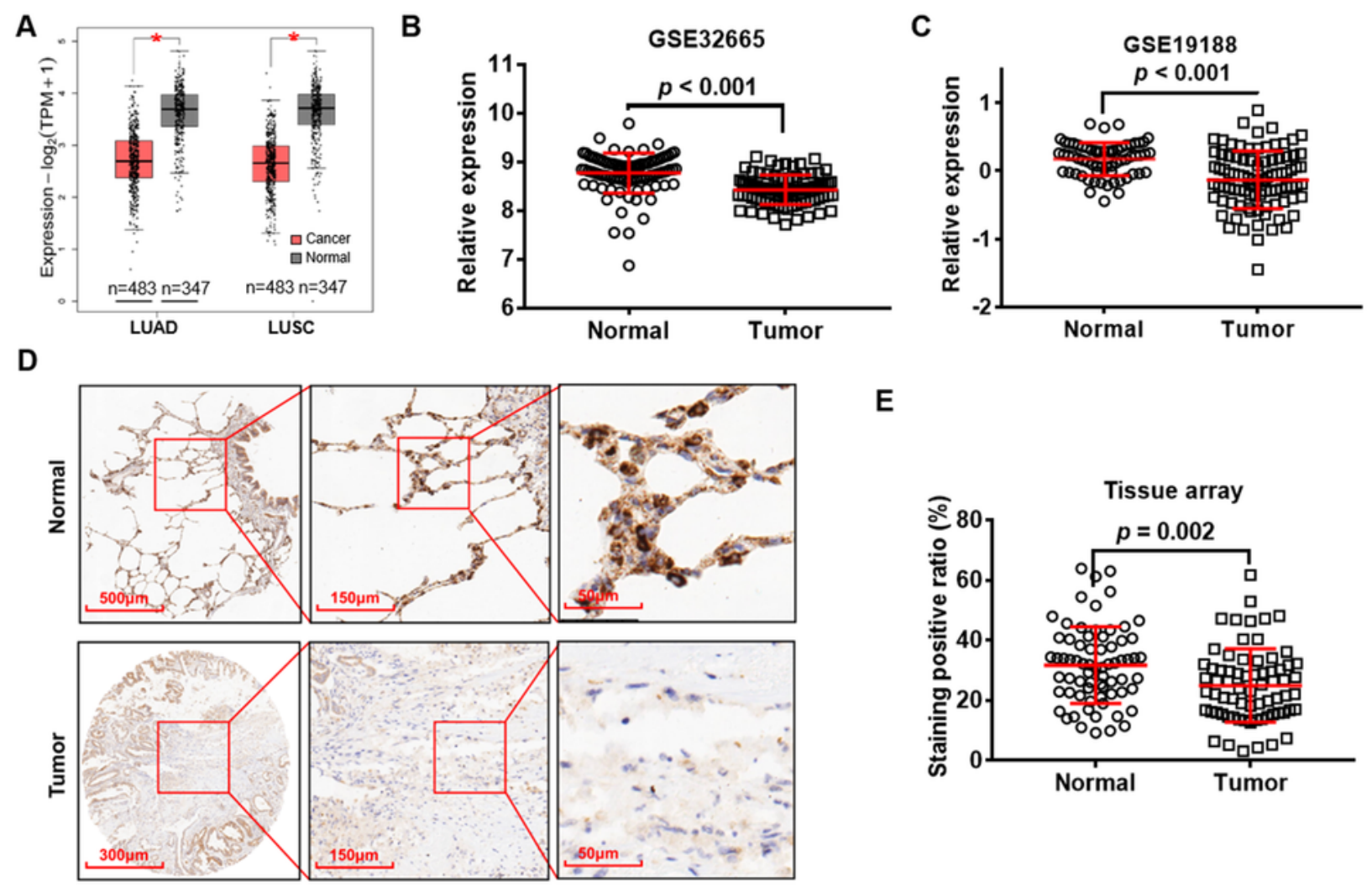

E
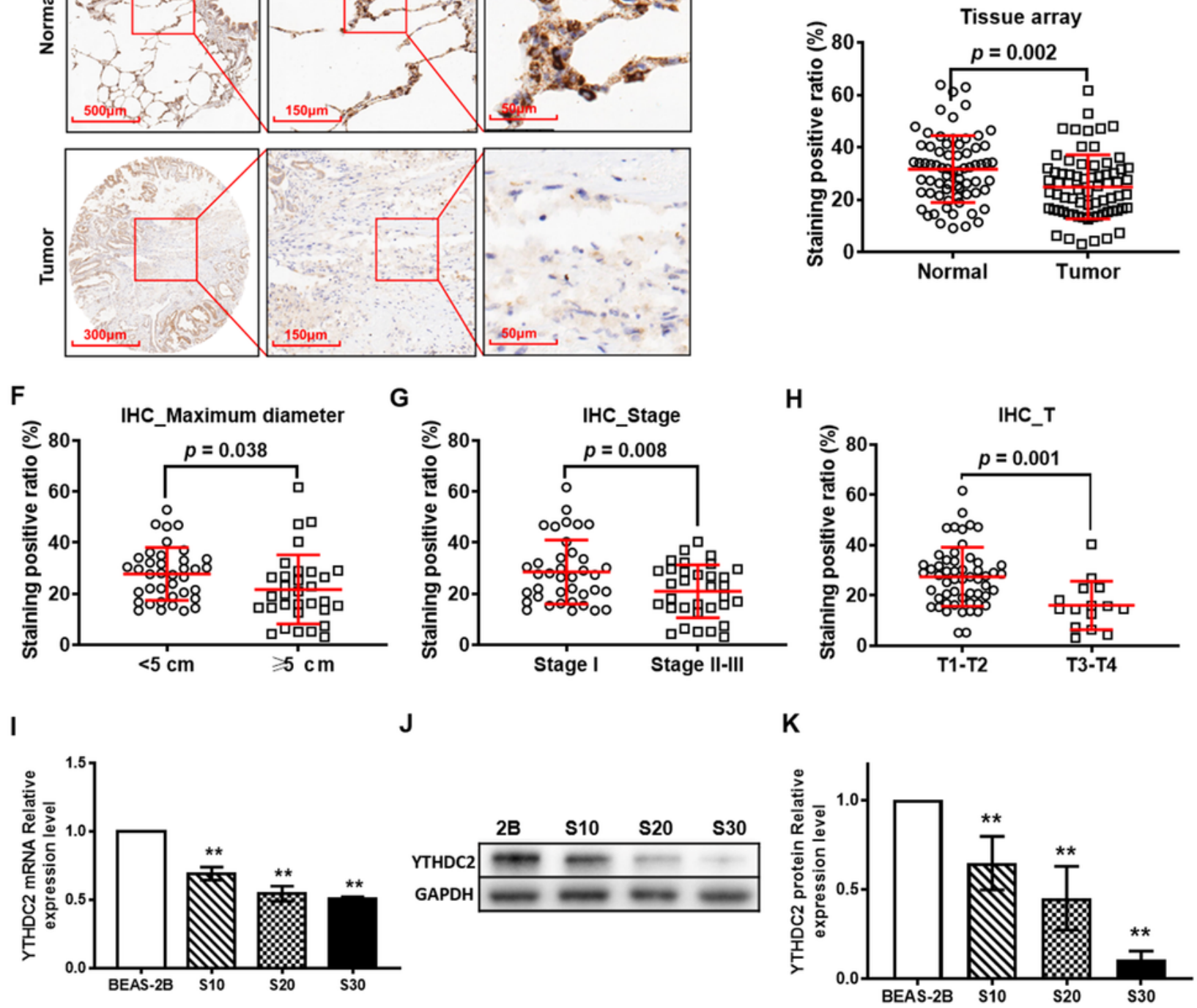

\section{Figure 1}

The YTHDC2 gene and protein were down-regulated in lung cancer patients from the TCGA, GEO and HPA databases, as well as in cigarette smoke-exposed cells. (A) Differential analysis of YTHDC2 mRNA expression in lung cancer tissues based on the GEPIA tool. ${ }^{*} p<0.05$ versus normal tissues. Differential analysis of YTHDC2 mRNA expression in lung cancer tissues in GSE32665 (B) and GSE19188 (C). (D) The representative IHC images showed that the YTHDC2 staining was found in the cytoplasm of the cells 
in lung cancer or normal lung tissues. High expression of YTHDC2 could be found in adjacent normal tissues, while its expression was decreased in most lung cancer tissues. (E) Differential analysis of YTHDC2 staining positive ratio quantitated by IHC profiler in lung cancer tissue arrays. (F) The relative mRNA expression level of YTHDC2 in cigarette smoke-exposed cells (S10, S20 and S30) and normal BEAS-2B cells. (G) Western blot analysis and quantitative results of YTHDC2 protein expression in cigarette smoke-exposed cells (S10, S20 and S30) and normal BEAS-2B cells. S10, S20 and S30 represent BEAS-2B cells exposed to cigarette smoke for 10,20 and 30 passages separately. ${ }^{*} p<0.01$ versus normal BEAS-2B cells. LUAD: lung adenocarcinoma; LUSC: Squamous cell carcinoma; IHC: Immunohistochemistry. 
A
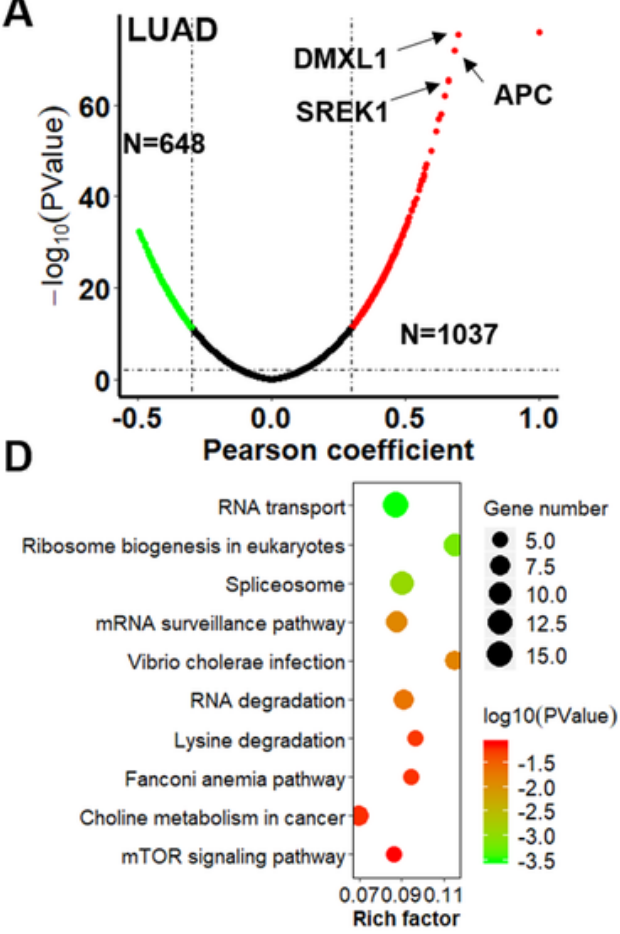

E
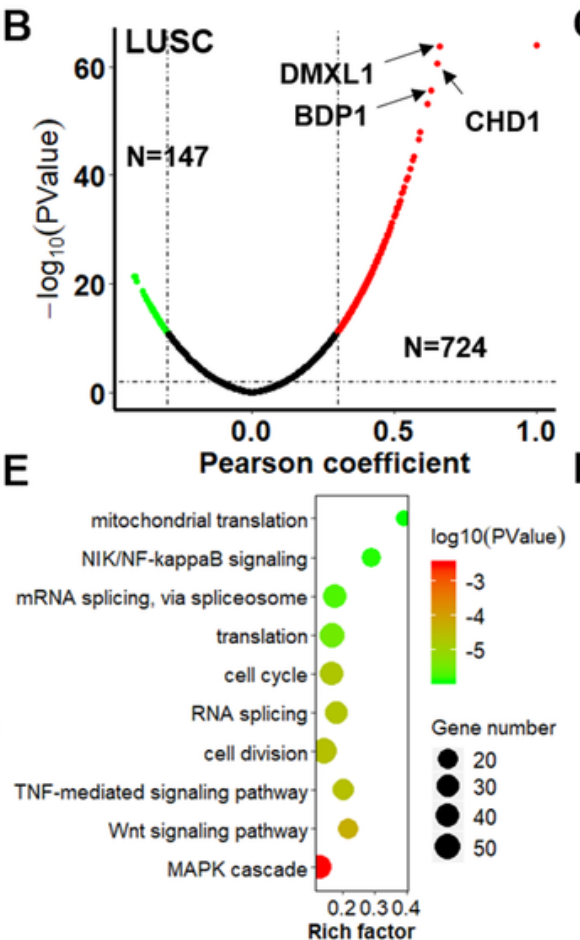

C

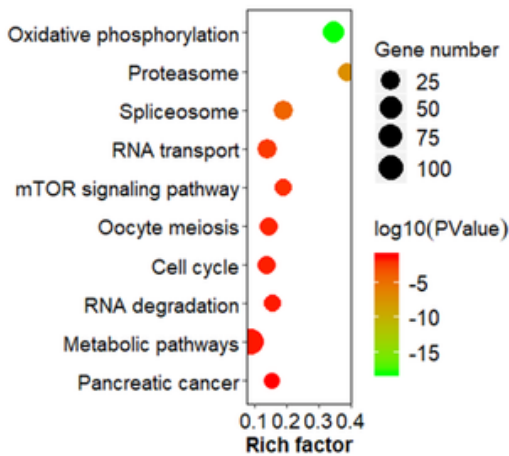

F
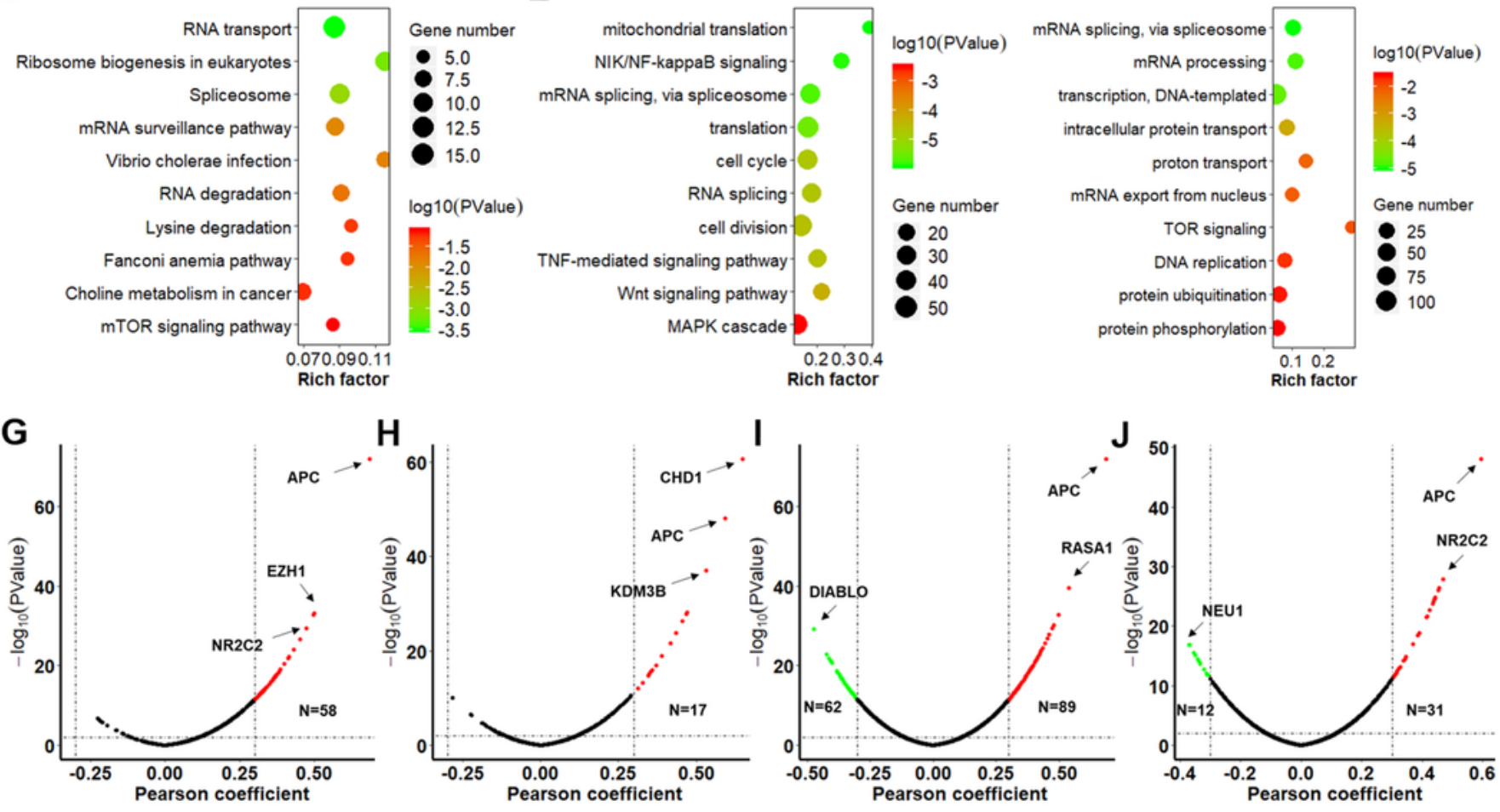

K
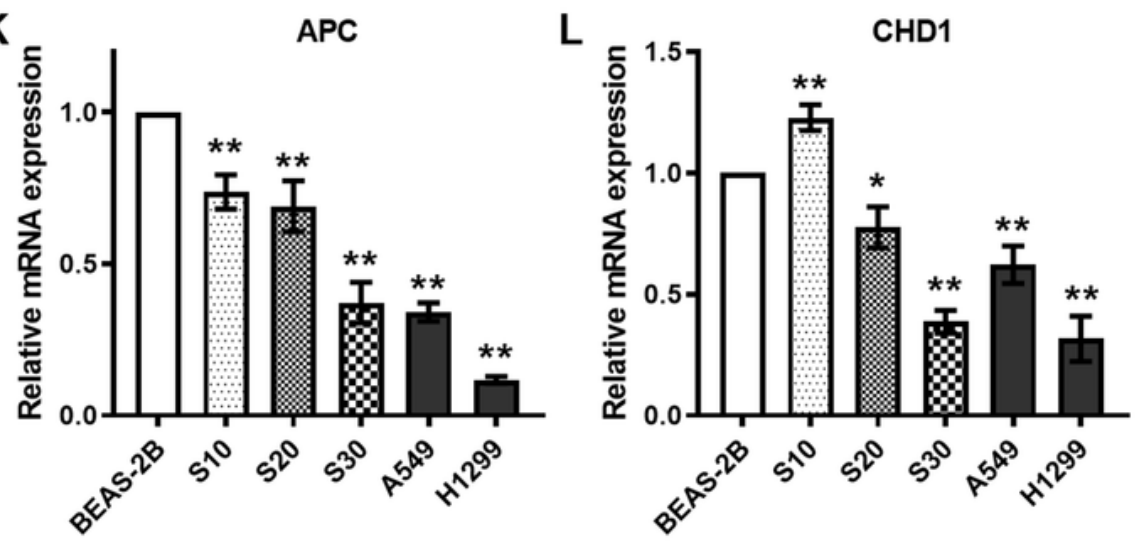

M

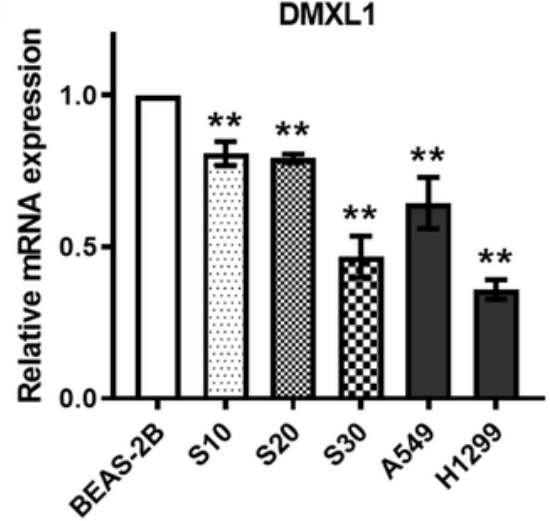

Figure 2

Identification of TSGs and MRGs in YTHDC2 related genes. Volcano plots showing the genes correlated with YTHDC2 in LUAD (A) and LUSC (B) in the TCGA database by using the LinkedOmics online tool. Scatter plots showing the KEGG enrichment results of the genes associated with LUAD (C) and LUSC (D). Scatter plots showing the biological process enrichment results of the genes associated with LUAD (E) and LUSC (F). Rich Factor is the ratio of the related genes annotated in a pathway to all the genes in this 
pathway. The larger the Rich factor, the greater the degree of enrichment. The color gradient from red to green represents the $\mathrm{p}$ value; the closer to green color, the lower the $\mathrm{p}$ value and the higher the significance level corresponding to the enrichment. Volcano plots showing the TSGs in YTHDC2 related genes in LUAD (G) and LUSC $(H)$. Volcano plots showing the MRGs in YTHDC2 related genes in LUAD (I) and LUSC $(J)$. The red dots in volcano plots represent the positively related genes (Pearson coefficient > $0.3, p<0.001$ ), and the green dots represent the negatively related genes (Pearson coefficient $<-0.3, p<$ 0.001), while the black dots represent genes have no significant correlation with YTHDC2 (Pearson coefficient between -0.3 and 0.3). The relative mRNA expression level of APC (K), CHD1 (L), DMXL1 (M) in cigarette smoke-exposed cells (S10, S20 and S30) and normal BEAS-2B cells. TSGs: Tumor suppressor genes; MRGs: Metastasis related genes; TCGA: The Cancer Gene Atlas; LUAD: Lung adenocarcinoma; LUSC: Lung squamous cell carcinoma; KEGG, Kyoto Encyclopedia of Genes and Genomes. 
A
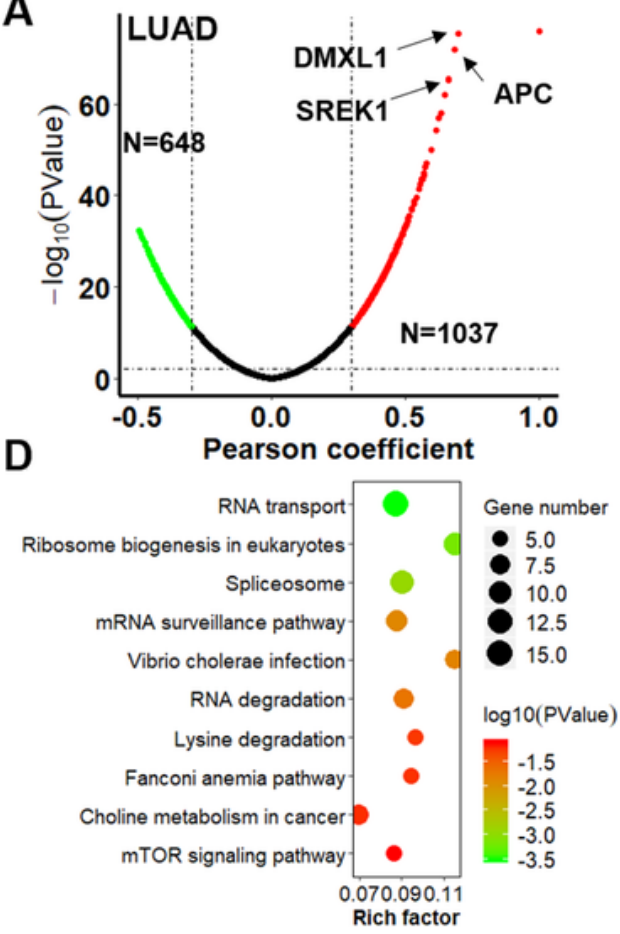

E
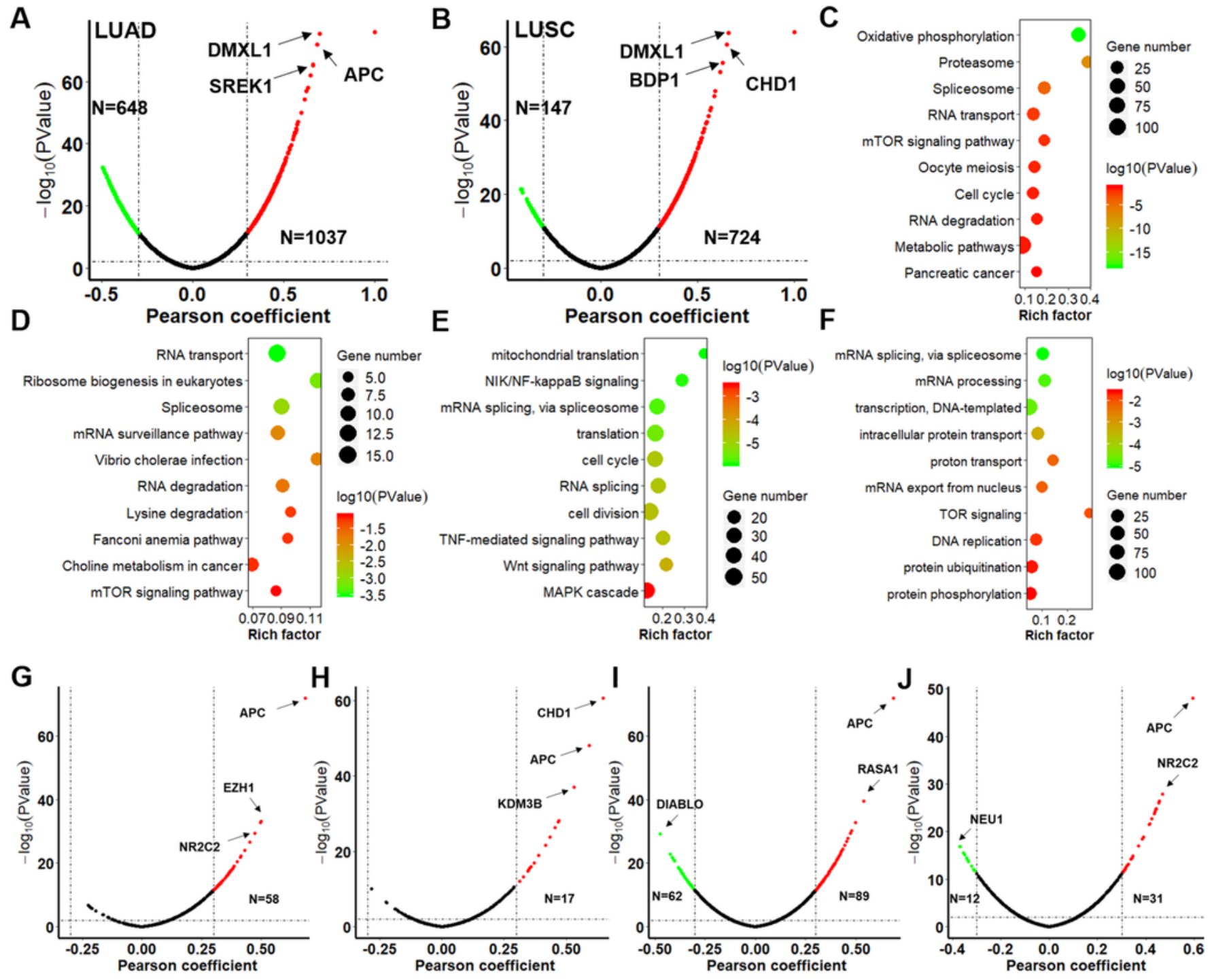

K
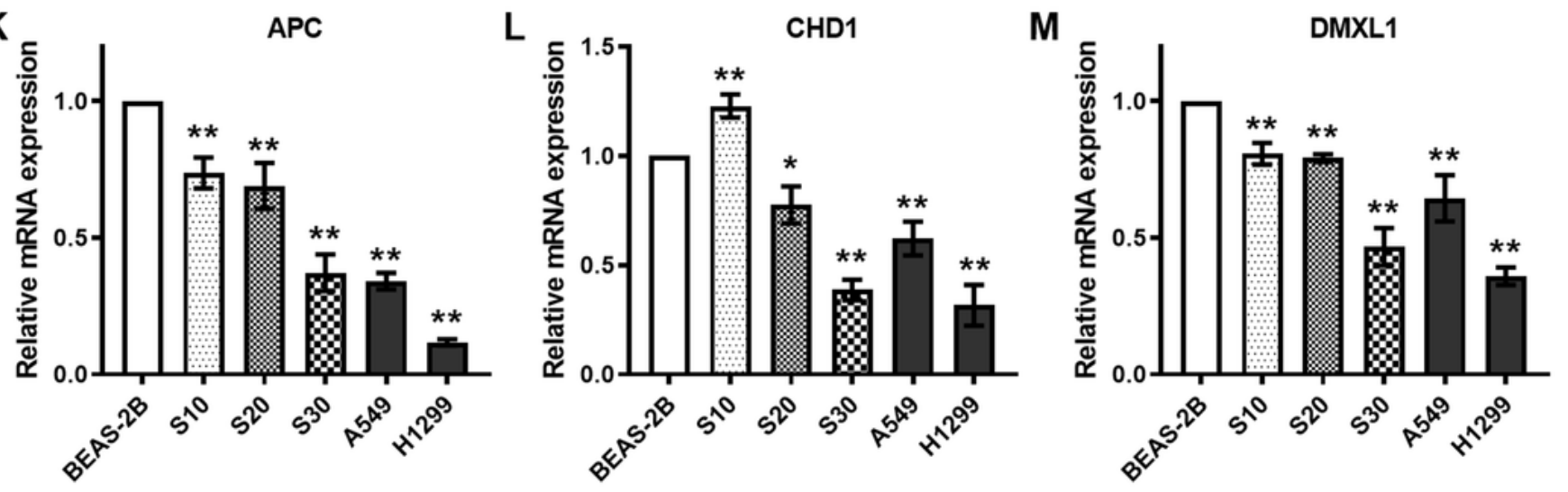

Figure 2

Identification of TSGs and MRGs in YTHDC2 related genes. Volcano plots showing the genes correlated with YTHDC2 in LUAD (A) and LUSC (B) in the TCGA database by using the LinkedOmics online tool. Scatter plots showing the KEGG enrichment results of the genes associated with LUAD (C) and LUSC (D). Scatter plots showing the biological process enrichment results of the genes associated with LUAD (E) and LUSC (F). Rich Factor is the ratio of the related genes annotated in a pathway to all the genes in this 
pathway. The larger the Rich factor, the greater the degree of enrichment. The color gradient from red to green represents the $\mathrm{p}$ value; the closer to green color, the lower the $\mathrm{p}$ value and the higher the significance level corresponding to the enrichment. Volcano plots showing the TSGs in YTHDC2 related genes in LUAD (G) and LUSC $(H)$. Volcano plots showing the MRGs in YTHDC2 related genes in LUAD (I) and LUSC $(J)$. The red dots in volcano plots represent the positively related genes (Pearson coefficient > $0.3, p<0.001$ ), and the green dots represent the negatively related genes (Pearson coefficient $<-0.3, p<$ 0.001), while the black dots represent genes have no significant correlation with YTHDC2 (Pearson coefficient between -0.3 and 0.3). The relative mRNA expression level of APC (K), CHD1 (L), DMXL1 (M) in cigarette smoke-exposed cells (S10, S20 and S30) and normal BEAS-2B cells. TSGs: Tumor suppressor genes; MRGs: Metastasis related genes; TCGA: The Cancer Gene Atlas; LUAD: Lung adenocarcinoma; LUSC: Lung squamous cell carcinoma; KEGG, Kyoto Encyclopedia of Genes and Genomes. 
A
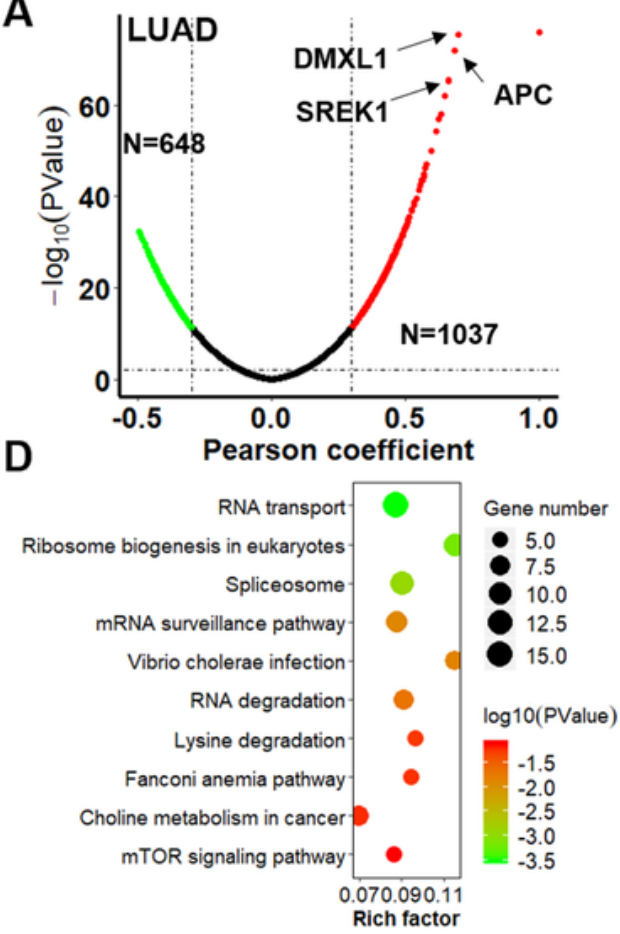

E
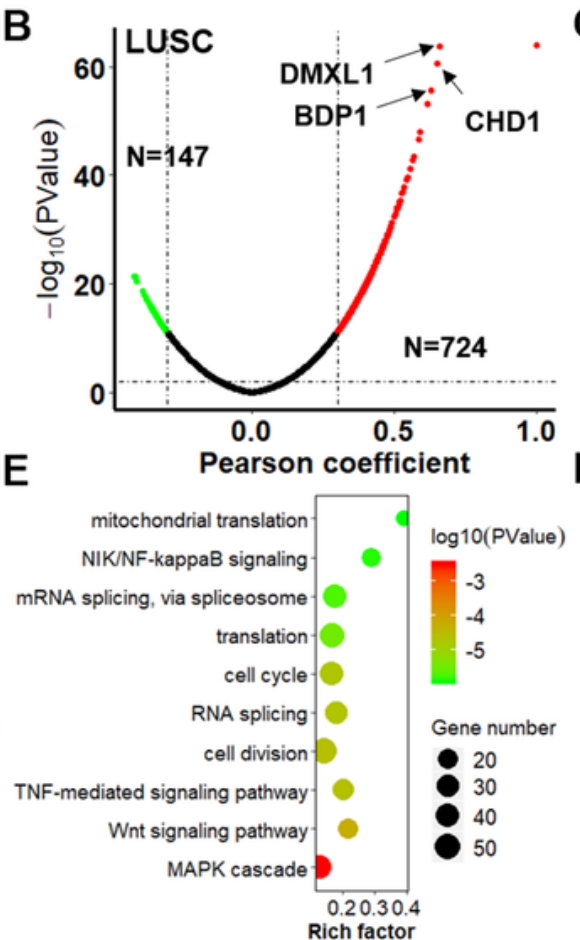

C

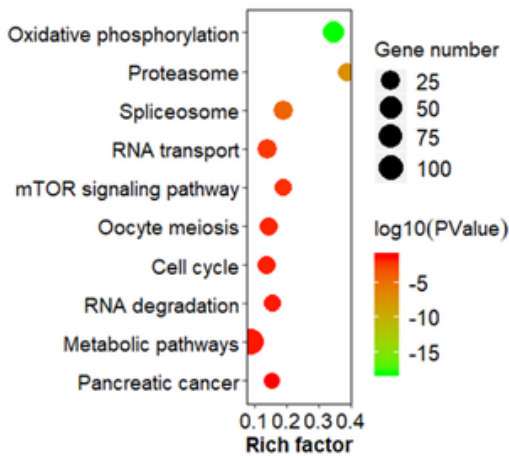

F
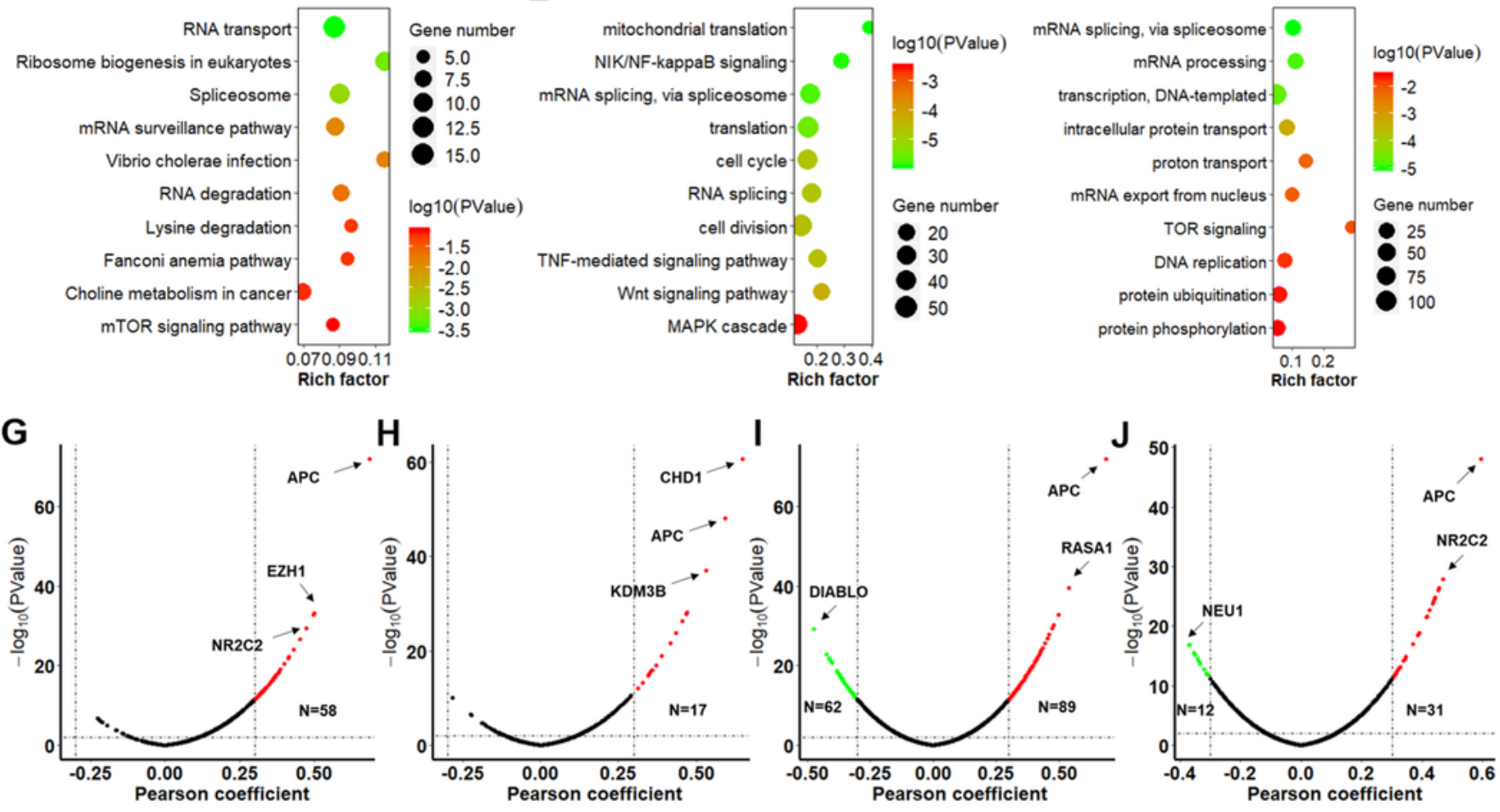

K
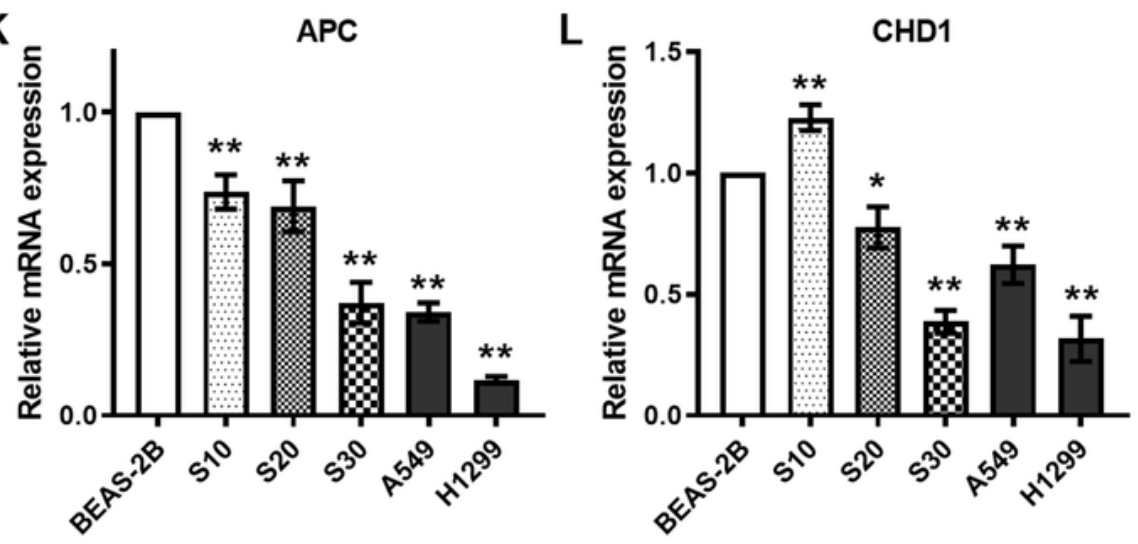

M

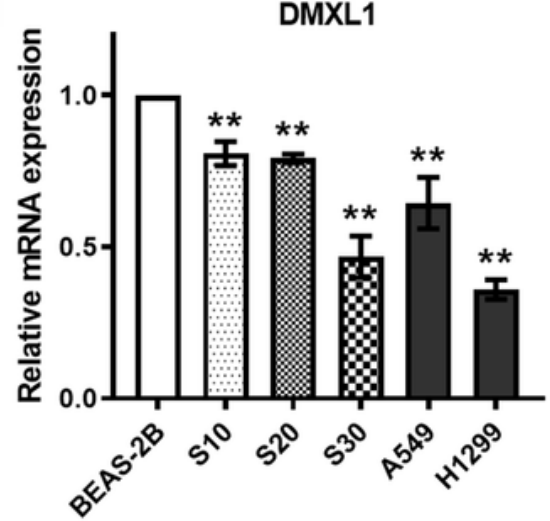

Figure 2

Identification of TSGs and MRGs in YTHDC2 related genes. Volcano plots showing the genes correlated with YTHDC2 in LUAD (A) and LUSC (B) in the TCGA database by using the LinkedOmics online tool. Scatter plots showing the KEGG enrichment results of the genes associated with LUAD (C) and LUSC (D). Scatter plots showing the biological process enrichment results of the genes associated with LUAD (E) and LUSC (F). Rich Factor is the ratio of the related genes annotated in a pathway to all the genes in this 
pathway. The larger the Rich factor, the greater the degree of enrichment. The color gradient from red to green represents the $\mathrm{p}$ value; the closer to green color, the lower the $\mathrm{p}$ value and the higher the significance level corresponding to the enrichment. Volcano plots showing the TSGs in YTHDC2 related genes in LUAD (G) and LUSC $(H)$. Volcano plots showing the MRGs in YTHDC2 related genes in LUAD (I) and LUSC $(J)$. The red dots in volcano plots represent the positively related genes (Pearson coefficient > $0.3, p<0.001$ ), and the green dots represent the negatively related genes (Pearson coefficient $<-0.3, p<$ 0.001), while the black dots represent genes have no significant correlation with YTHDC2 (Pearson coefficient between -0.3 and 0.3). The relative mRNA expression level of APC (K), CHD1 (L), DMXL1 (M) in cigarette smoke-exposed cells (S10, S20 and S30) and normal BEAS-2B cells. TSGs: Tumor suppressor genes; MRGs: Metastasis related genes; TCGA: The Cancer Gene Atlas; LUAD: Lung adenocarcinoma; LUSC: Lung squamous cell carcinoma; KEGG, Kyoto Encyclopedia of Genes and Genomes. 
A

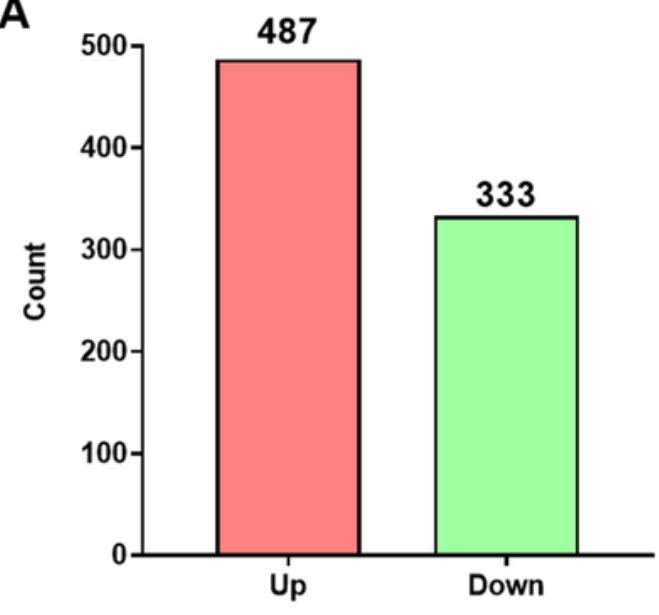

C

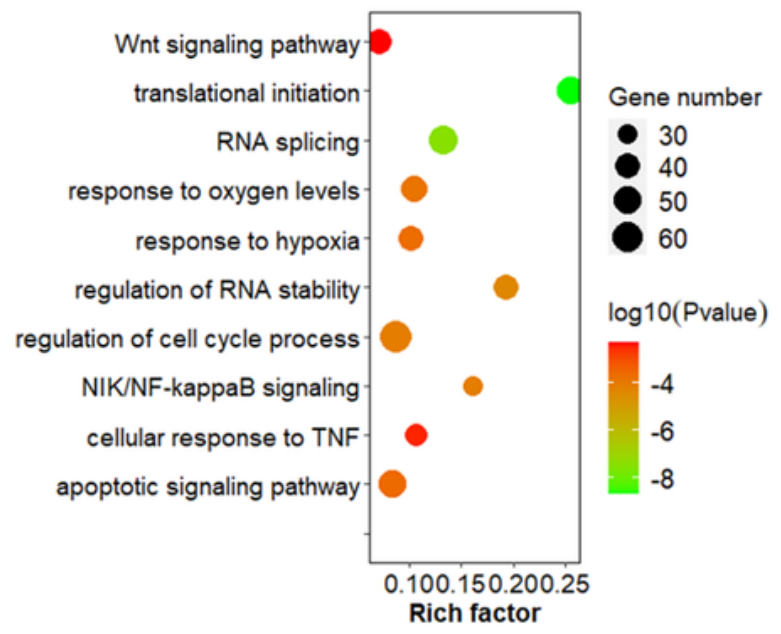

B

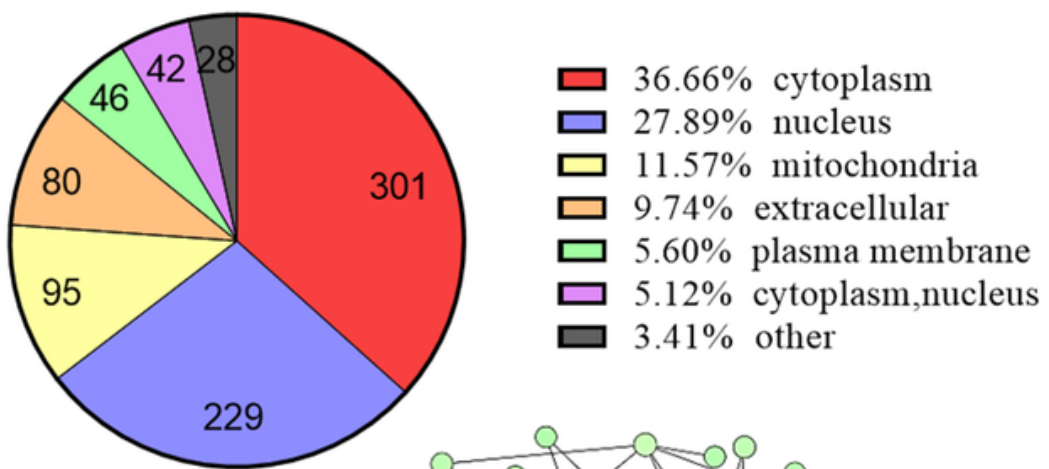

E

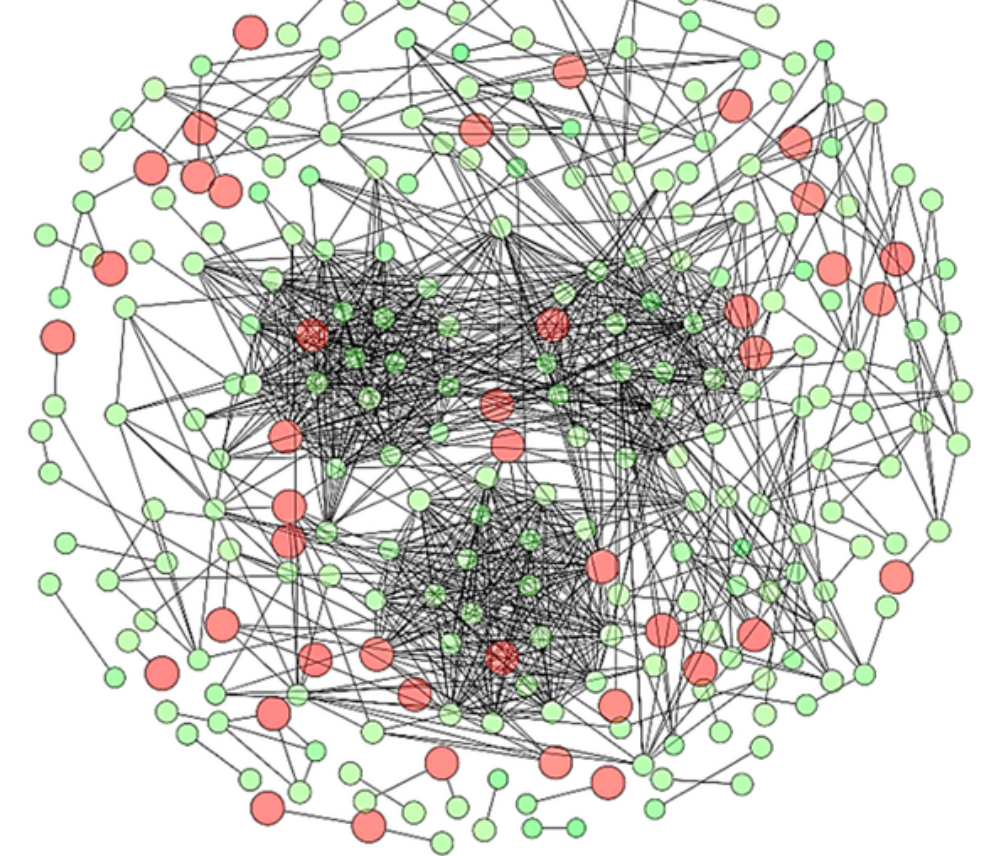

$\mathrm{g} H \mathrm{H} 16 \mathrm{SF}$
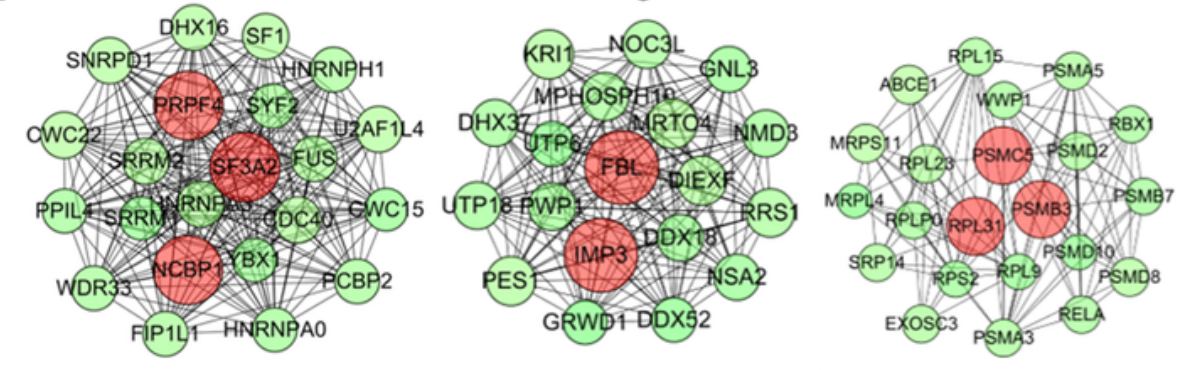

\begin{tabular}{lcll}
\hline Description & $\begin{array}{c}\text { Log10 } \\
\text { (P value) }\end{array}$ & & Desc \\
\cline { 1 - 2 } ribosome biogenesis & -20.40 & & reg \\
ncRNA processing & -14.63 & W \\
RNA localization & -3.426 & ce
\end{tabular}

\begin{tabular}{lc}
\hline Description & \multicolumn{1}{c}{$\begin{array}{l}\text { Log10 } \\
\text { (P value) }\end{array}$} \\
\hline regulation of RNA stability & -13.68 \\
Wnt signaling pathway & -9.66 \\
cell cycle phase transition & -8.90 \\
\hline
\end{tabular}

\section{Figure 3}

Proteomics analysis of YTHDC2 knockdown cells. (A) Bar graph showing the number of up-regulated and downregulated proteins in YTHDC2 knockdown cells. (B) Subcellular distribution of differentially expressed proteins (DEGs). Biological process (C) and KEGG pathway (D) analysis of DEPs. (E) The PPI network of DEGs was constructed using Cytoscape. (F) The top 3 significant clusters were identified by plugin MCODE in Cytoscape, and the biological process enrichment analysis of the proteins in these 
clusters. PPI, Protein-protein interaction; KEGG, Kyoto Encyclopedia of Genes and Genomes; DEPs, differentially expressed proteins.

A

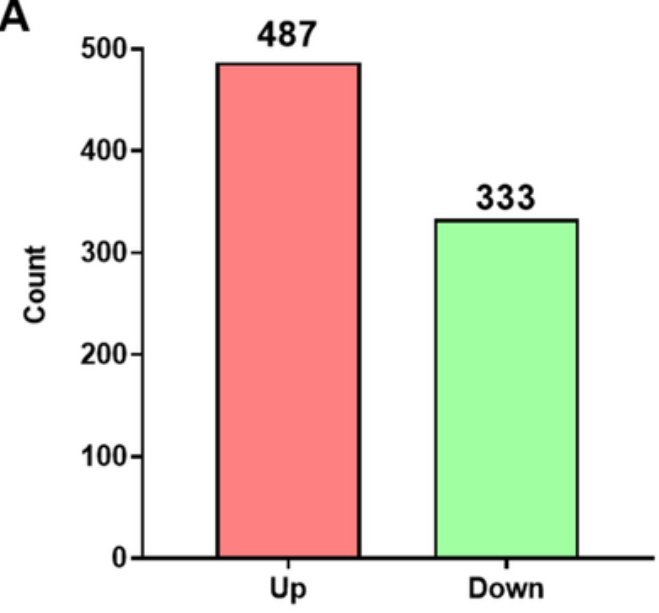

C

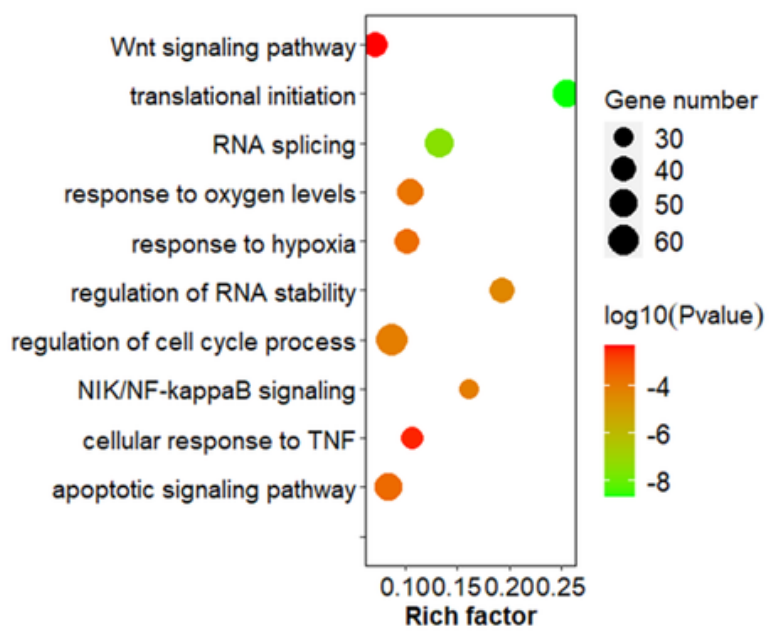

B
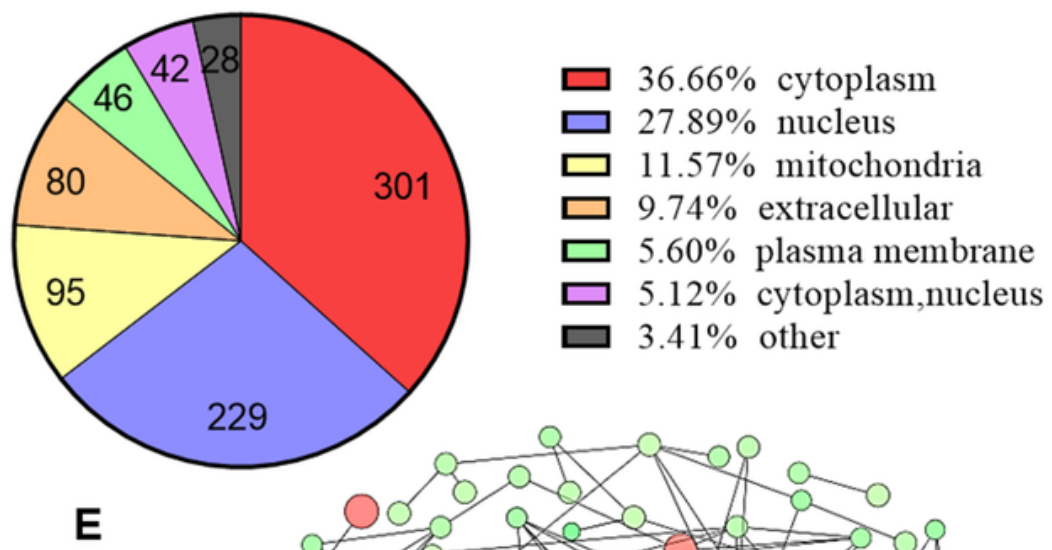

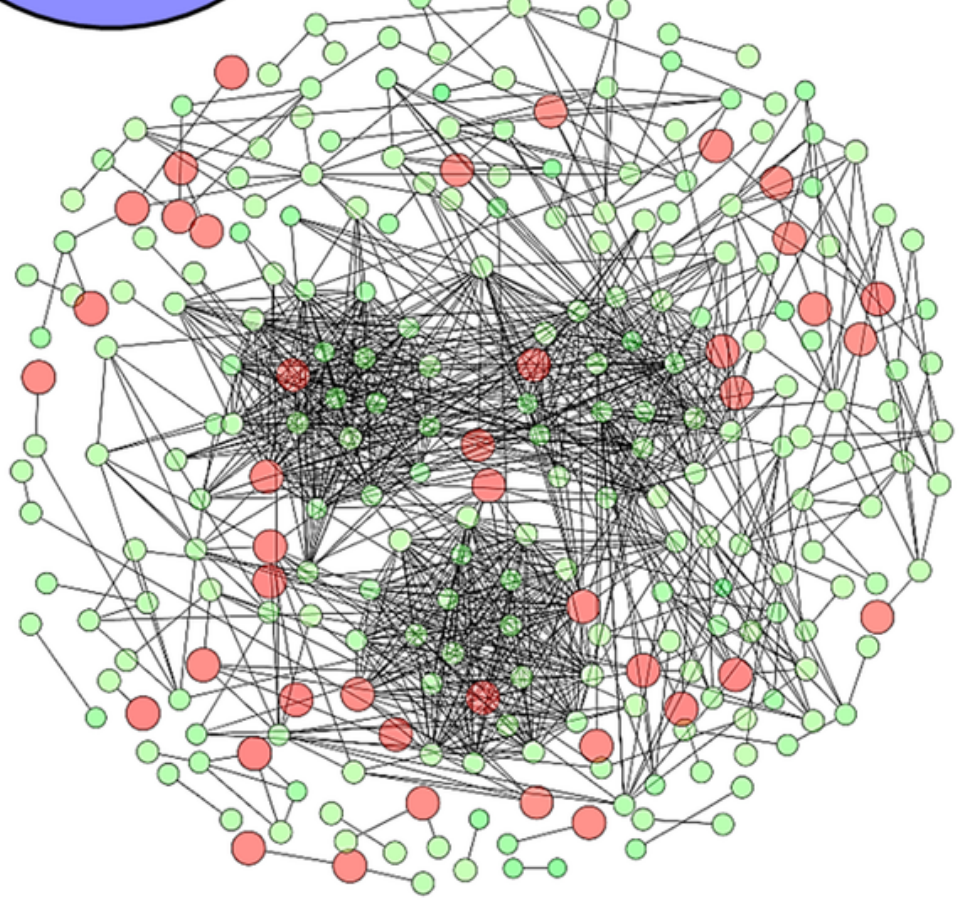

D

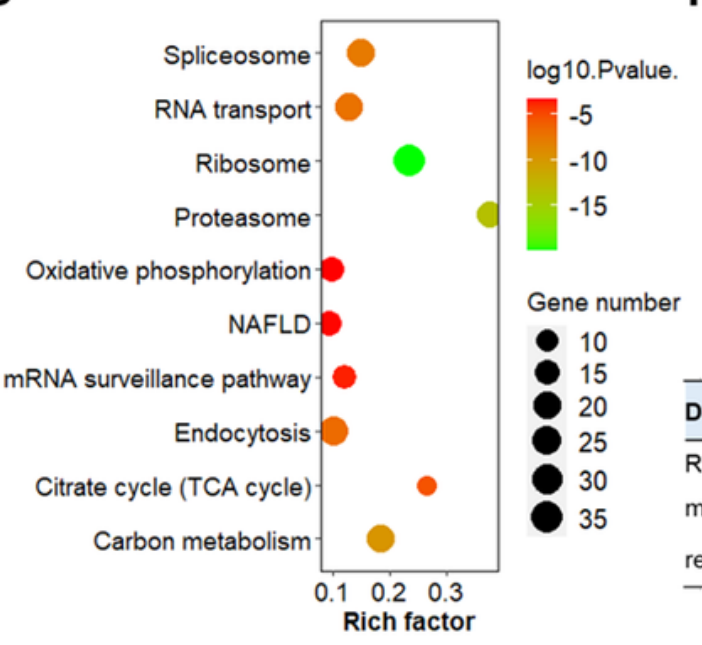

$\mathbf{F}$
gHX16 SF1)

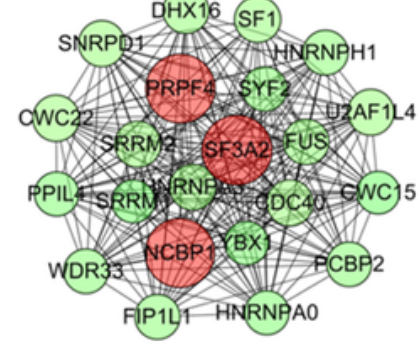

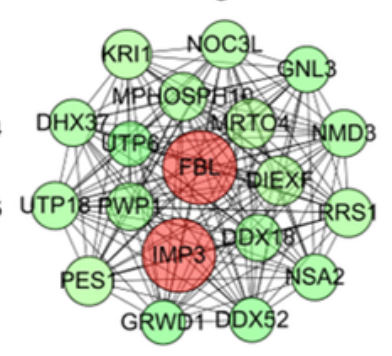

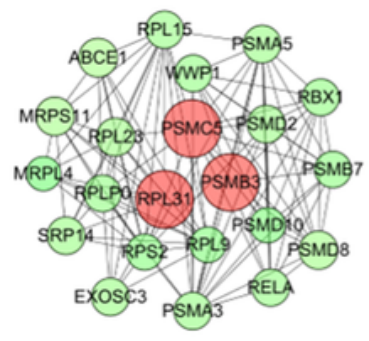

\begin{tabular}{|c|c|c|c|c|c|}
\hline Description & $\begin{array}{l}\text { Log10 } \\
\text { (P value) }\end{array}$ & Description & $\begin{array}{l}\text { Log10 } \\
\text { (P value) }\end{array}$ & Description & $\begin{array}{l}\text { Log10 } \\
\text { (P value) }\end{array}$ \\
\hline RNA splicing & -35.55 & ribosome biogenesis & -20.40 & regulation of RNA stability & -13.68 \\
\hline mRNA processing & -34.14 & ncRNA processing & -14.63 & Wnt signaling pathway & -9.66 \\
\hline regulation of RNA stability & -3.211 & RNA localization & -3.426 & cell cycle phase transition & -8.90 \\
\hline
\end{tabular}

\section{Figure 3}

Proteomics analysis of YTHDC2 knockdown cells. (A) Bar graph showing the number of up-regulated and downregulated proteins in YTHDC2 knockdown cells. (B) Subcellular distribution of differentially expressed proteins (DEGs). Biological process (C) and KEGG pathway (D) analysis of DEPs. (E) The PPI 
network of DEGs was constructed using Cytoscape. (F) The top 3 significant clusters were identified by plugin MCODE in Cytoscape, and the biological process enrichment analysis of the proteins in these clusters. PPI, Protein-protein interaction; KEGG, Kyoto Encyclopedia of Genes and Genomes; DEPs, differentially expressed proteins.

A

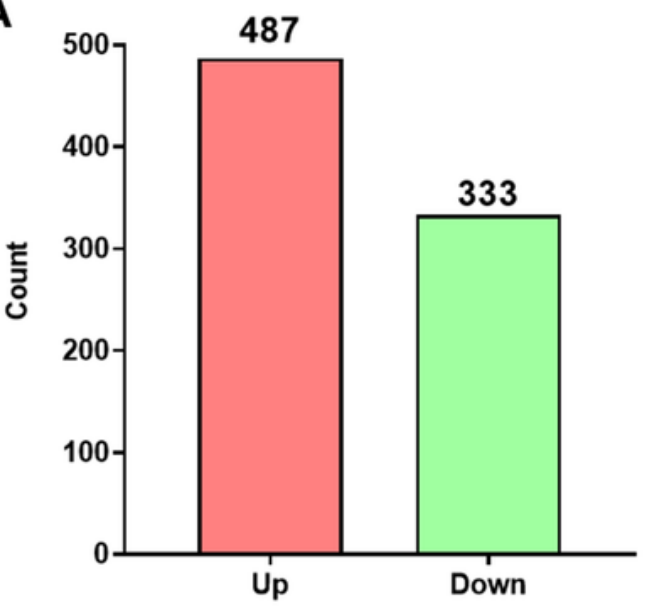

C

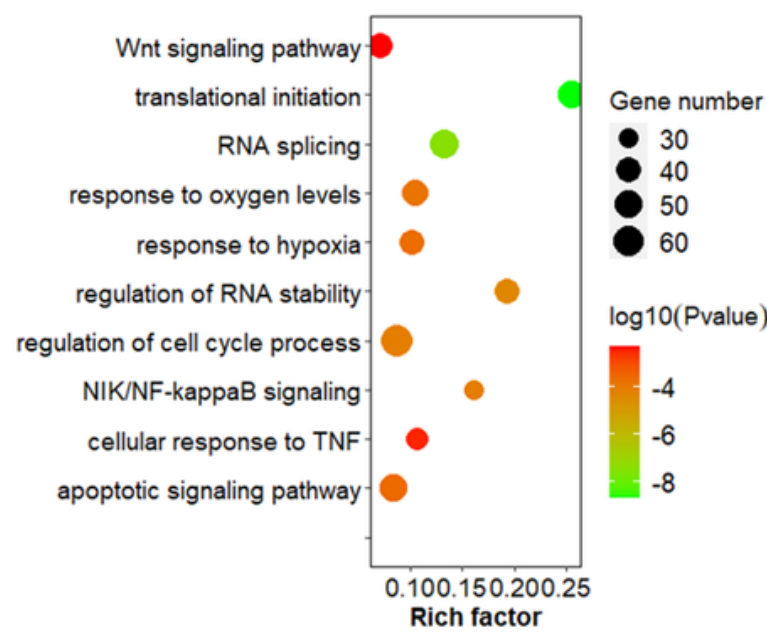

B

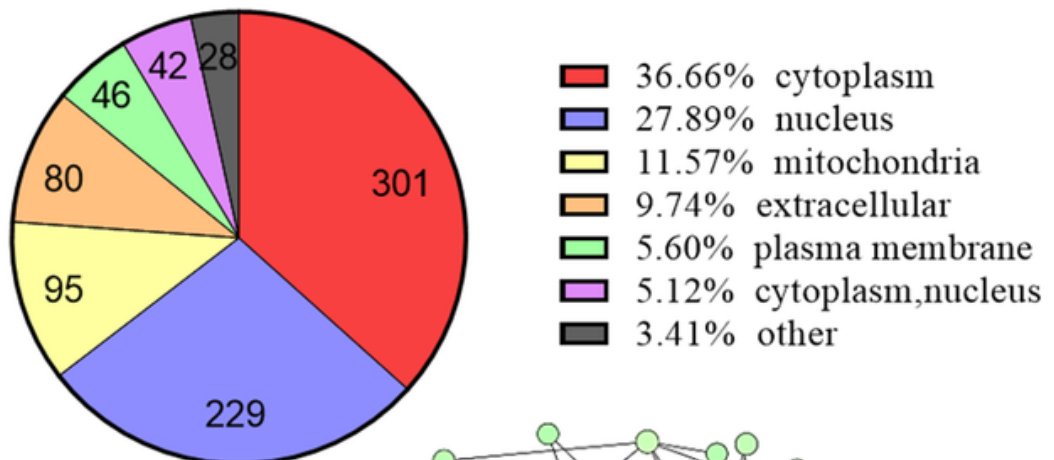

E

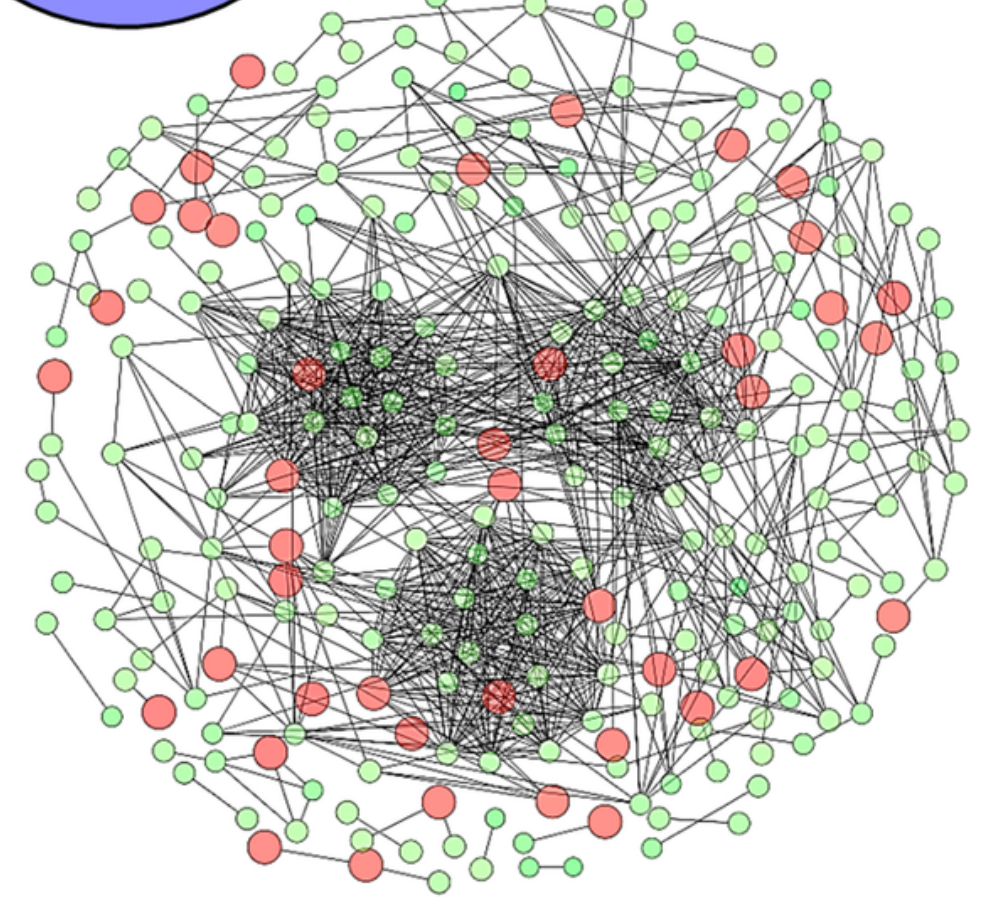

$\mathbf{F}$
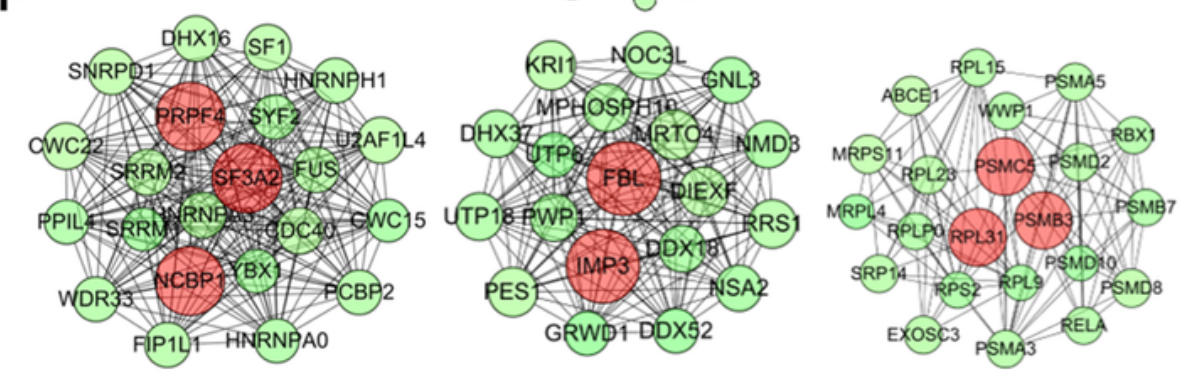

\begin{tabular}{|c|c|c|c|c|c|}
\hline Description & $\begin{array}{l}\text { Log10 } \\
\text { (P value) }\end{array}$ & Description & $\begin{array}{l}\text { Log10 } \\
\text { (P value) }\end{array}$ & Description & $\begin{array}{l}\text { Log10 } \\
\text { (P value) }\end{array}$ \\
\hline RNA splicing & -35.55 & ribosome biogenesis & -20.40 & regulation of RNA stability & -13.68 \\
\hline mRNA processing & -34.14 & ncRNA processing & -14.63 & Wnt signaling pathway & -9.66 \\
\hline regulation of RNA stability & -3.211 & RNA localization & -3.426 & cell cycle phase transition & -8.90 \\
\hline
\end{tabular}

Figure 3 
Proteomics analysis of YTHDC2 knockdown cells. (A) Bar graph showing the number of up-regulated and downregulated proteins in YTHDC2 knockdown cells. (B) Subcellular distribution of differentially expressed proteins (DEGs). Biological process (C) and KEGG pathway (D) analysis of DEPs. (E) The PPI network of DEGs was constructed using Cytoscape. $(F)$ The top 3 significant clusters were identified by plugin MCODE in Cytoscape, and the biological process enrichment analysis of the proteins in these clusters. PPI, Protein-protein interaction; KEGG, Kyoto Encyclopedia of Genes and Genomes; DEPs, differentially expressed proteins.
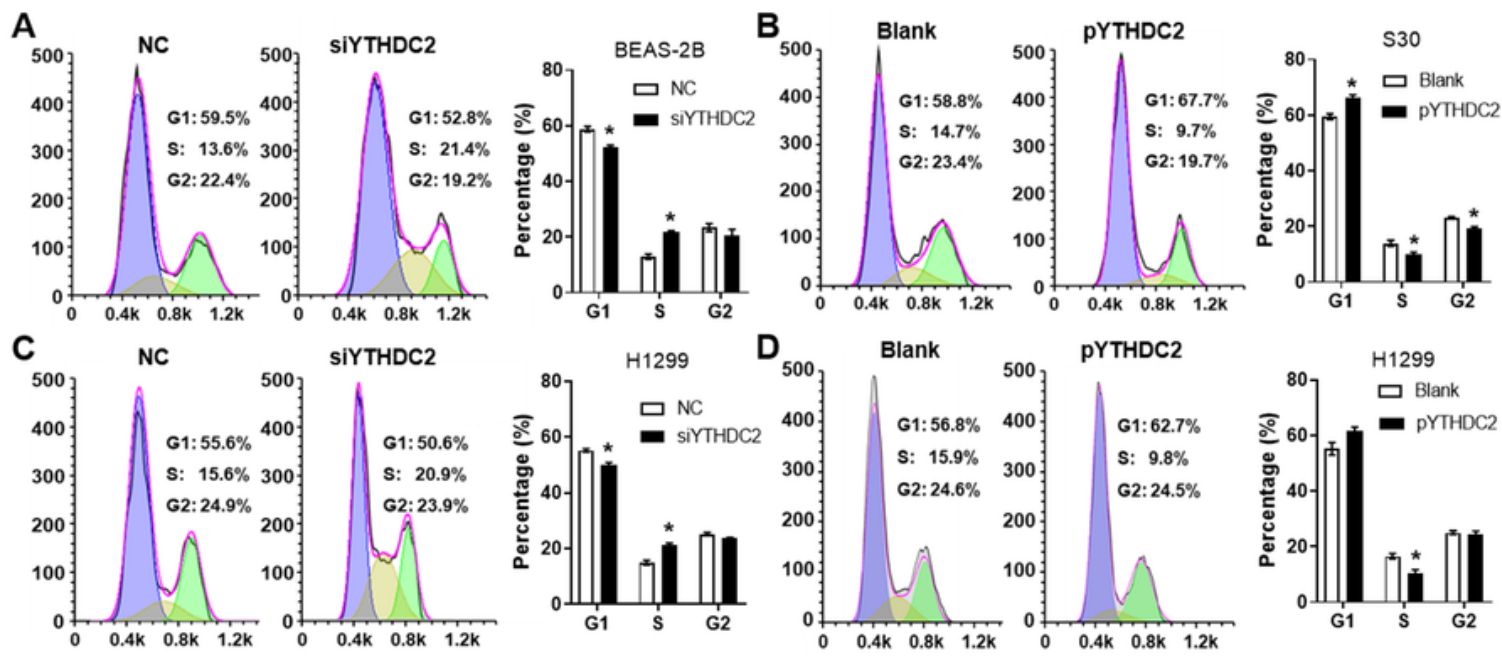

E

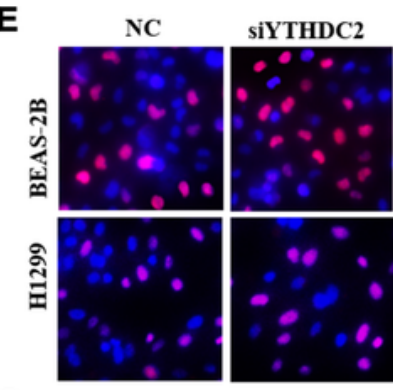

G

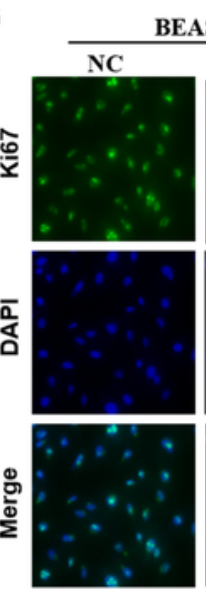

I
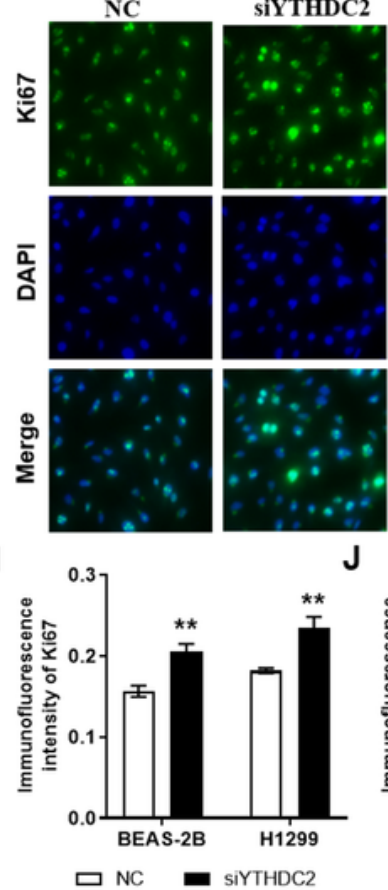

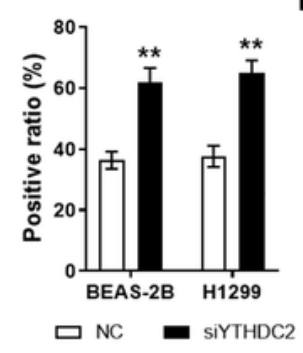

F

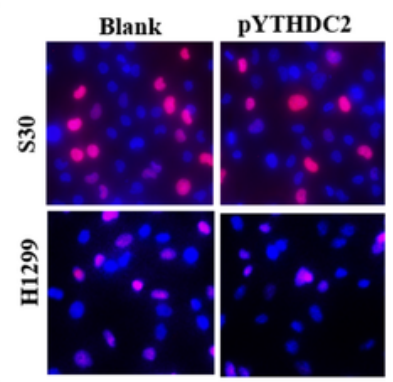

$\mathrm{H}$
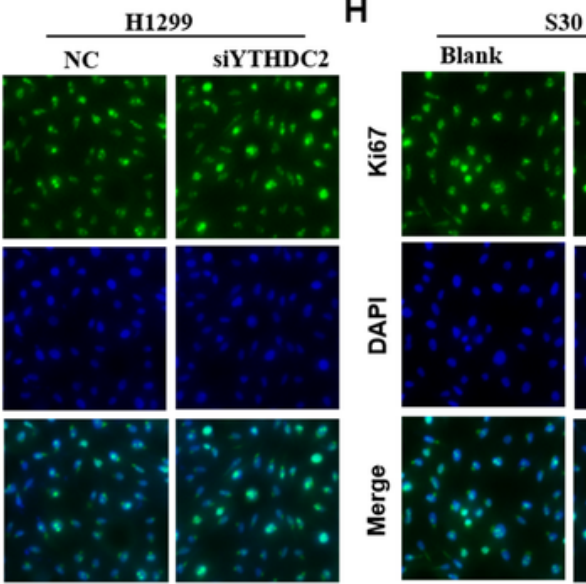

PYTHDC2

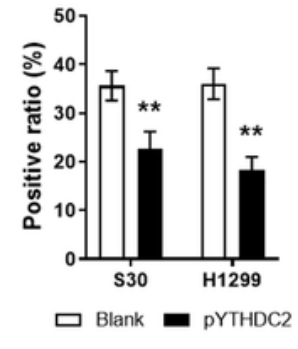

K
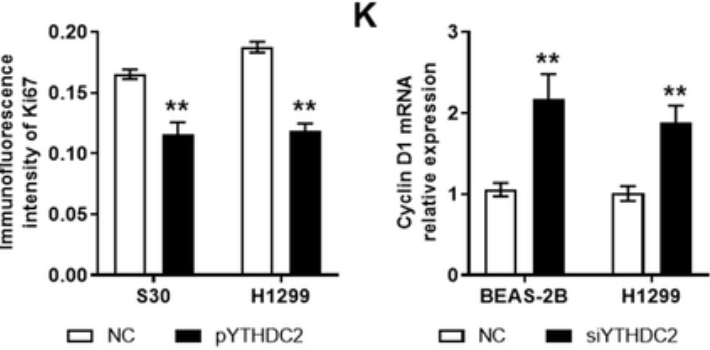

L
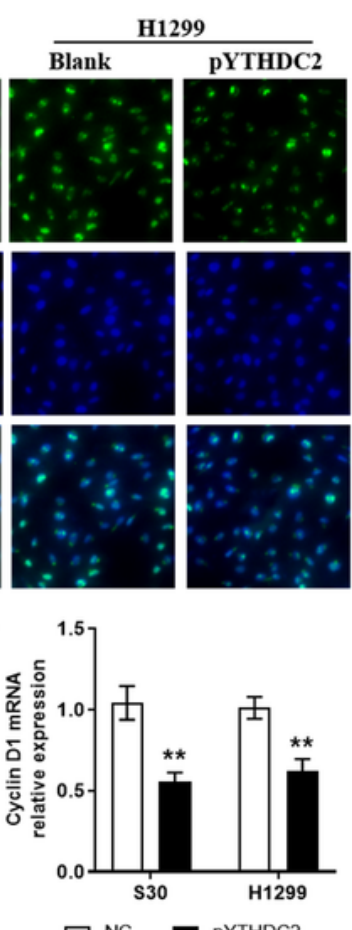

口 NC DYTHDC2 


\section{Figure 4}

YTHDC2 downregulation promotes lung cancer cell proliferation. Representative images and quantification results of cell cycle of BEAS-2B (A) and H1299 (C) cells transfected with small interfering RNA (siYTHDC2) and negative control (NC). Representative images and quantification results of cell cycle of S30 (B) and H1299 (D) cells transfected with over-expression vector (pYTHDC2) and blank vector. (E) Cell proliferation were measured in BEAS-2B and H1299 cells transfected with siYTHDC2 and NC by Edu cell proliferation assay. (F) Cell proliferation were measured in $\mathrm{S} 30$ and $\mathrm{H} 1299$ cells transfected with pYTHDC2 and Blank by Edu cell proliferation assay. Representative immunofluorescence images $(\mathrm{G})$ and quantification results (I) of Ki67 in BEAS-2B and H1299 cells transfected with siYTHDC2 and NC. Representative immunofluorescence images $(\mathrm{H})$ and quantification results $(\mathrm{J})$ of Ki67 in $\mathrm{S} 30$ and $\mathrm{H} 1299$ cells transfected with pYTHDC2 and Blank. The relative mRNA expression level of Cyclin D1 in BEAS-2B and $\mathrm{H} 1299$ cells transfected with siYTHDC2 and $\mathrm{NC}(\mathrm{K})$, as well as in $\mathrm{S} 30$ and $\mathrm{H} 1299$ cells transfected with pYTHDC2 and Blank (L). *p $<0.05$ vs. NC (Blank) group; ${ }^{* \star} p<0.01$ vs. NC (Blank) group. 

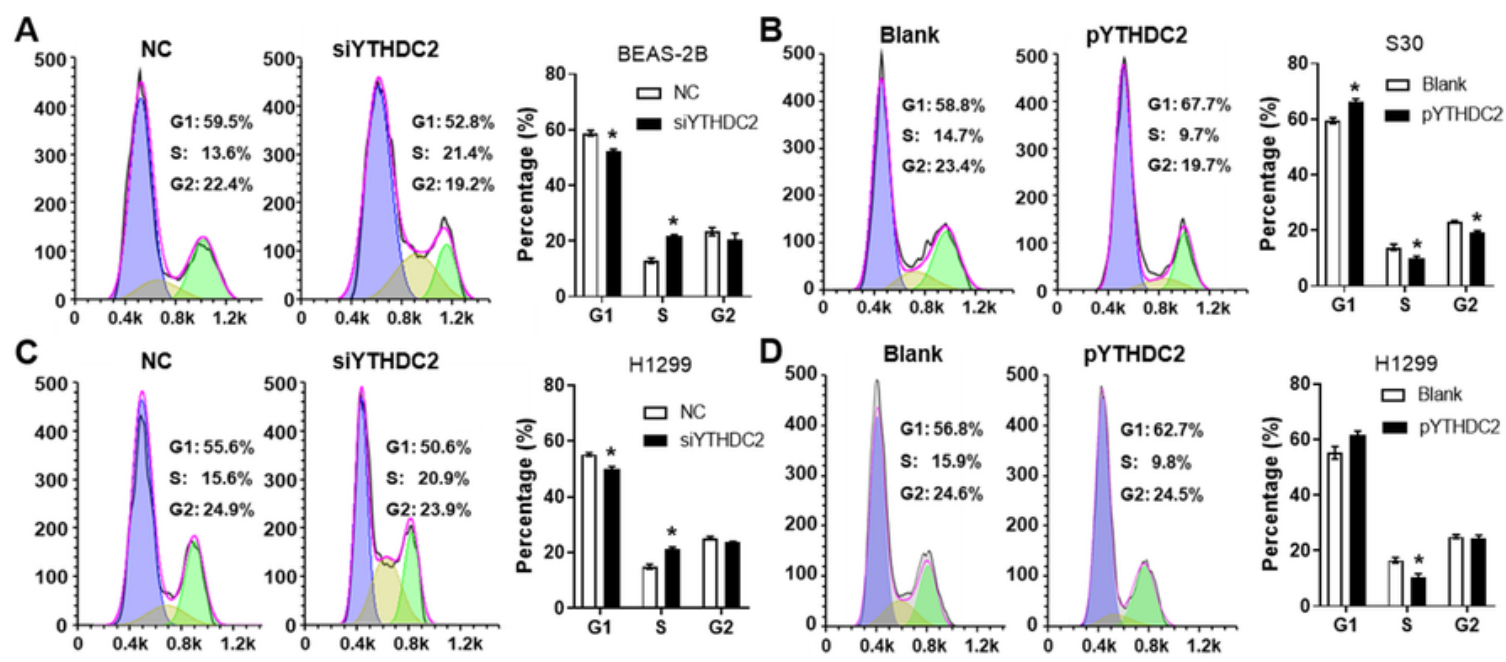

E
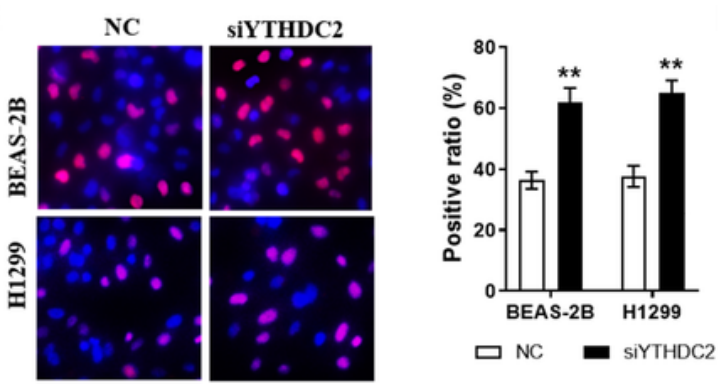

$\mathbf{F}$
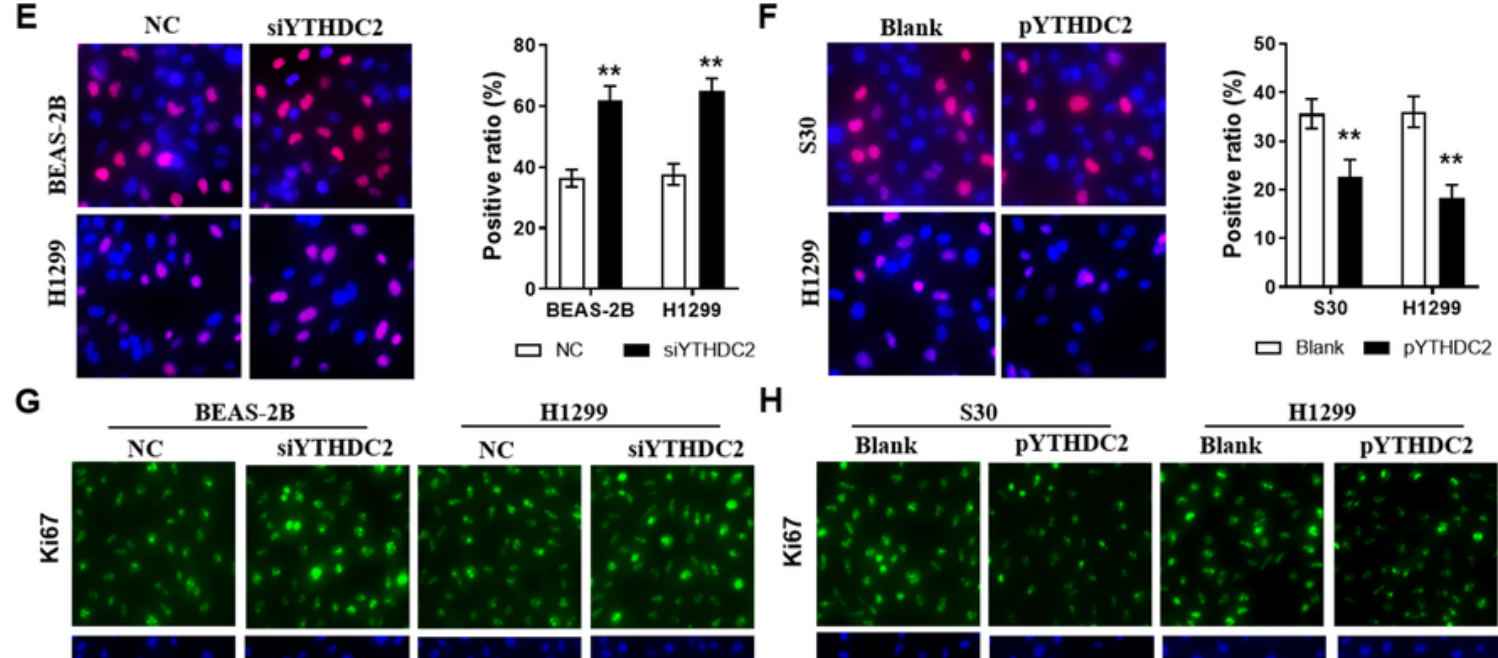

$\mathrm{H}$
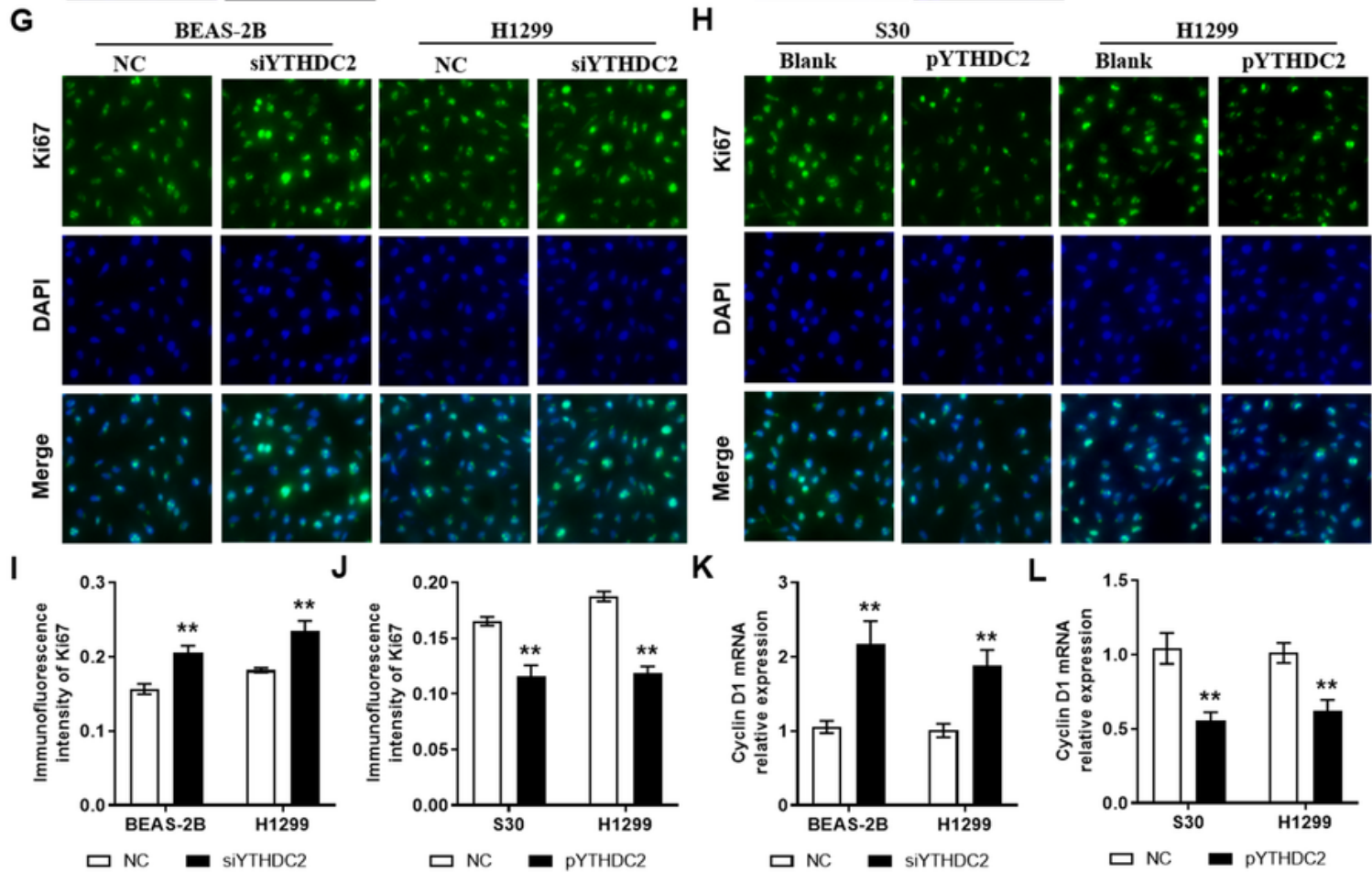

K
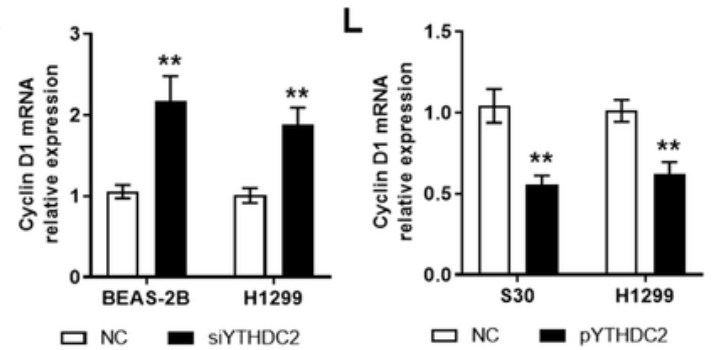

Figure 4

YTHDC2 downregulation promotes lung cancer cell proliferation. Representative images and quantification results of cell cycle of BEAS-2B (A) and H1299 (C) cells transfected with small interfering RNA (siYTHDC2) and negative control (NC). Representative images and quantification results of cell cycle of S30 (B) and H1299 (D) cells transfected with over-expression vector (pYTHDC2) and blank vector. (E) Cell proliferation were measured in BEAS-2B and H1299 cells transfected with siYTHDC2 and NC by Edu 
cell proliferation assay. (F) Cell proliferation were measured in S30 and H1299 cells transfected with pYTHDC2 and Blank by Edu cell proliferation assay. Representative immunofluorescence images $(\mathrm{G})$ and quantification results (I) of Ki67 in BEAS-2B and H1299 cells transfected with siYTHDC2 and NC.

Representative immunofluorescence images $(\mathrm{H})$ and quantification results $(\mathrm{J})$ of Ki67 in S30 and H1299 cells transfected with pYTHDC2 and Blank. The relative mRNA expression level of Cyclin D1 in BEAS-2B and $\mathrm{H} 1299$ cells transfected with siYTHDC2 and $\mathrm{NC}(\mathrm{K})$, as well as in $\mathrm{S} 30$ and $\mathrm{H} 1299$ cells transfected with pYTHDC2 and Blank (L). *p $<0.05$ vs. NC (Blank) group; **p $<0.01$ vs. NC (Blank) group.
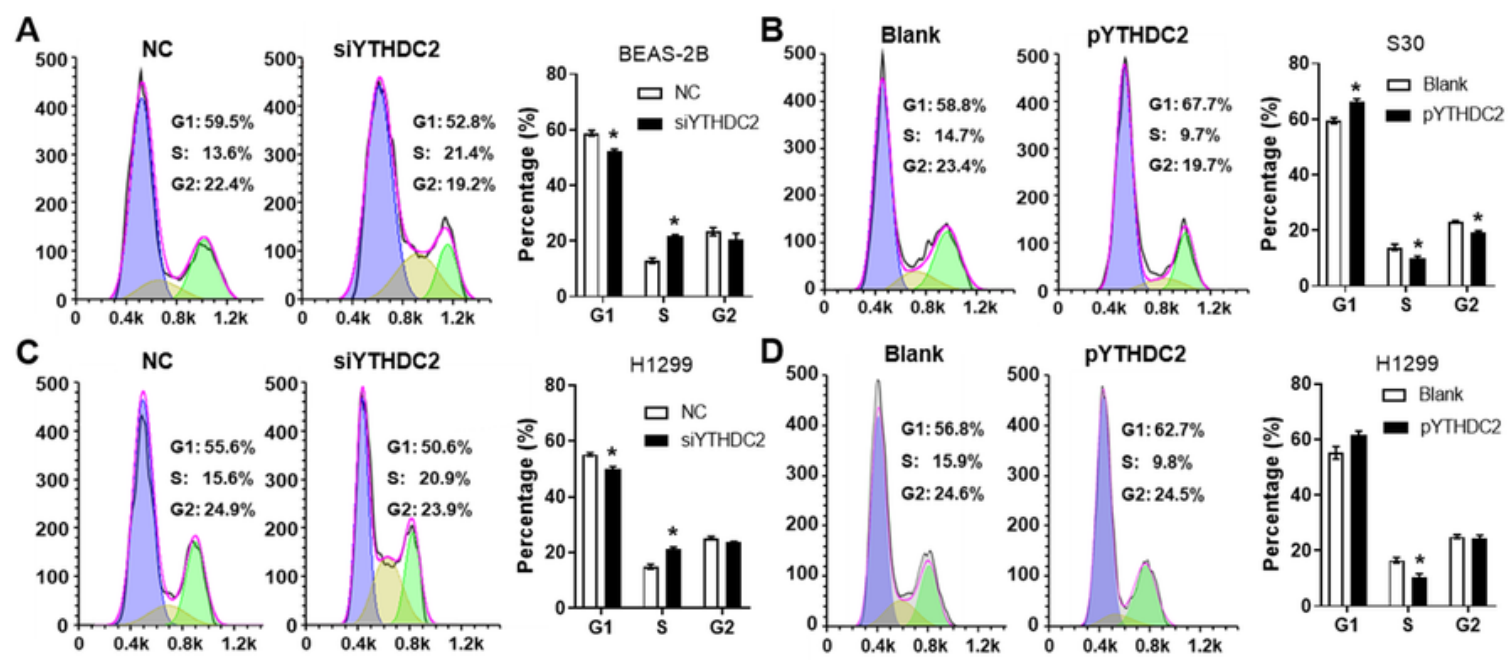

E

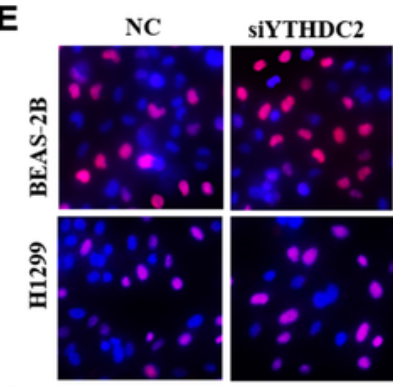

G

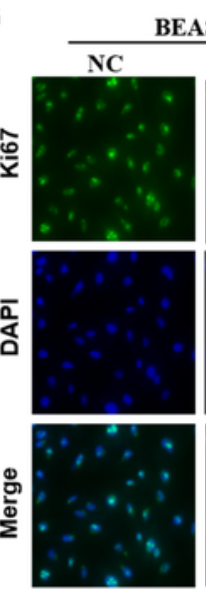

I

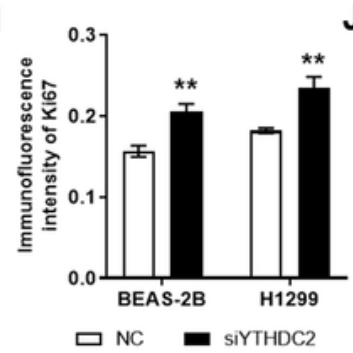

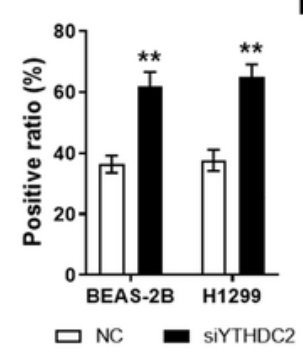

$\mathbf{F}$
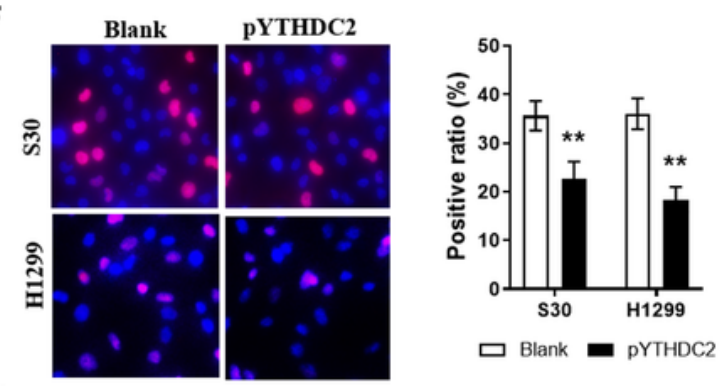

$\mathrm{H}$
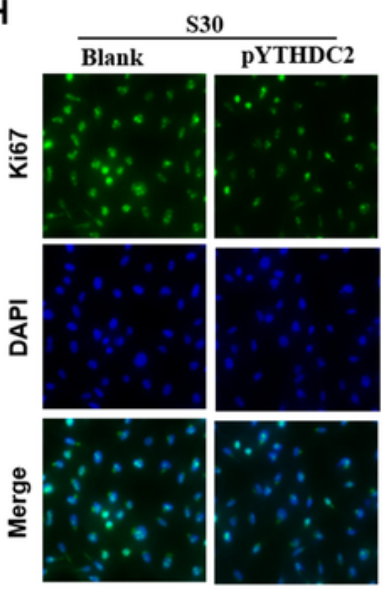

K

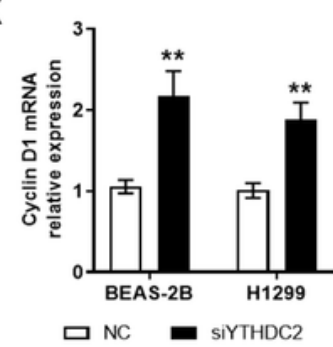

L

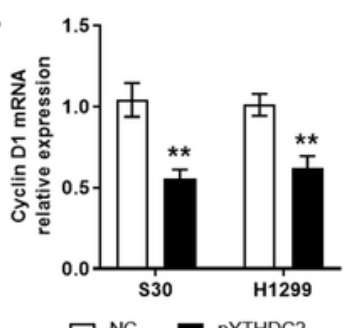




\section{Figure 4}

YTHDC2 downregulation promotes lung cancer cell proliferation. Representative images and quantification results of cell cycle of BEAS-2B (A) and H1299 (C) cells transfected with small interfering RNA (siYTHDC2) and negative control (NC). Representative images and quantification results of cell cycle of S30 (B) and H1299 (D) cells transfected with over-expression vector (pYTHDC2) and blank vector. (E) Cell proliferation were measured in BEAS-2B and H1299 cells transfected with siYTHDC2 and NC by Edu cell proliferation assay. (F) Cell proliferation were measured in $\mathrm{S} 30$ and $\mathrm{H} 1299$ cells transfected with pYTHDC2 and Blank by Edu cell proliferation assay. Representative immunofluorescence images $(\mathrm{G})$ and quantification results (I) of Ki67 in BEAS-2B and H1299 cells transfected with siYTHDC2 and NC. Representative immunofluorescence images $(\mathrm{H})$ and quantification results $(\mathrm{J})$ of Ki67 in $\mathrm{S} 30$ and $\mathrm{H} 1299$ cells transfected with pYTHDC2 and Blank. The relative mRNA expression level of Cyclin D1 in BEAS-2B and $\mathrm{H} 1299$ cells transfected with siYTHDC2 and $\mathrm{NC}(\mathrm{K})$, as well as in $\mathrm{S} 30$ and $\mathrm{H} 1299$ cells transfected with pYTHDC2 and Blank (L). *p $<0.05$ vs. NC (Blank) group; ${ }^{* \star} p<0.01$ vs. NC (Blank) group. 
A

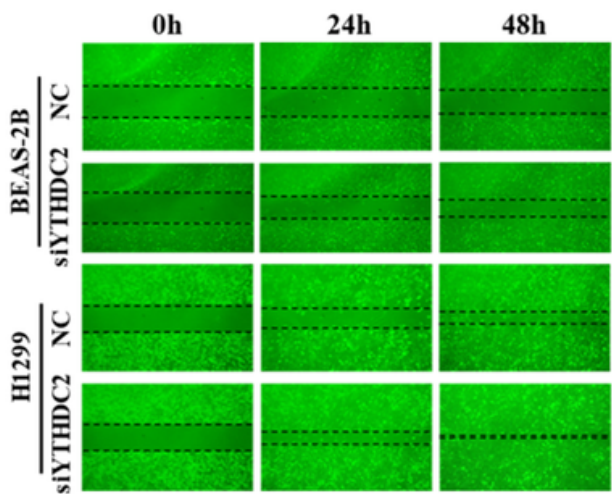

B
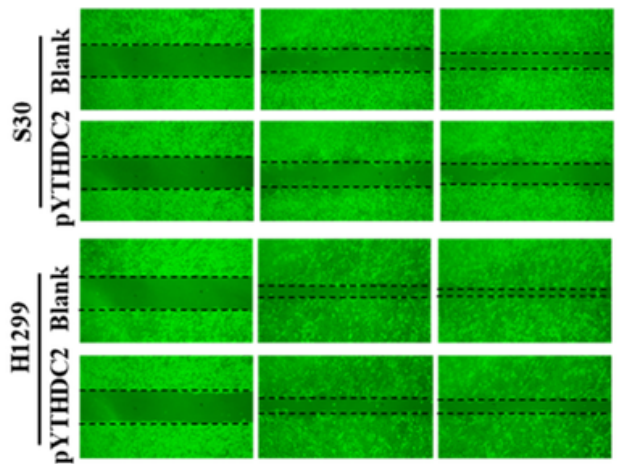

C
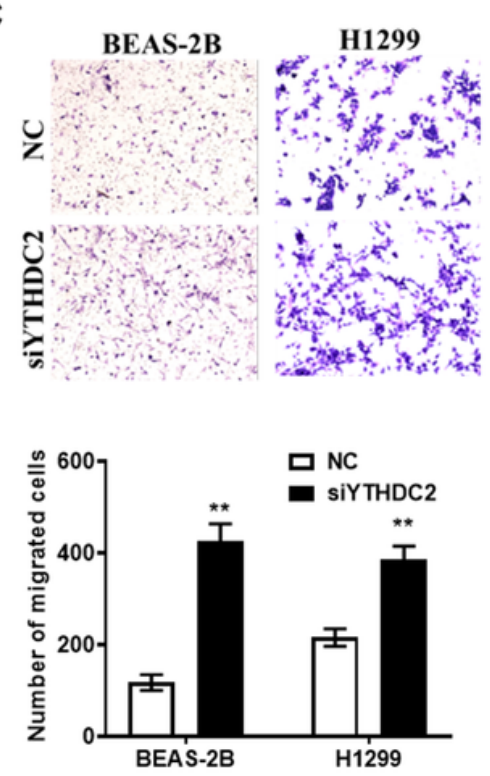
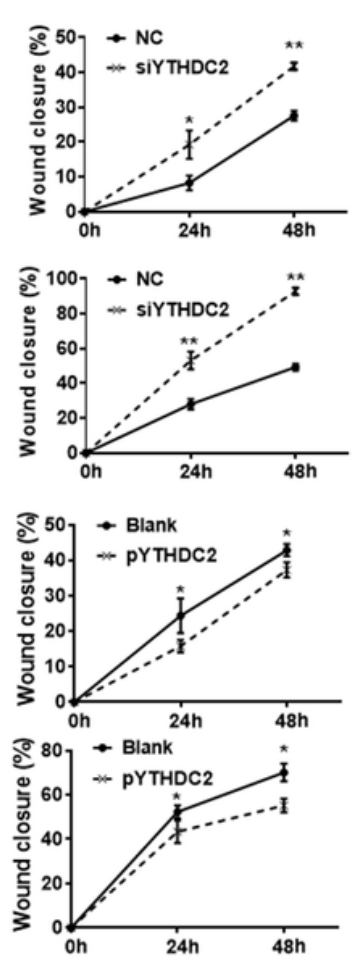

D
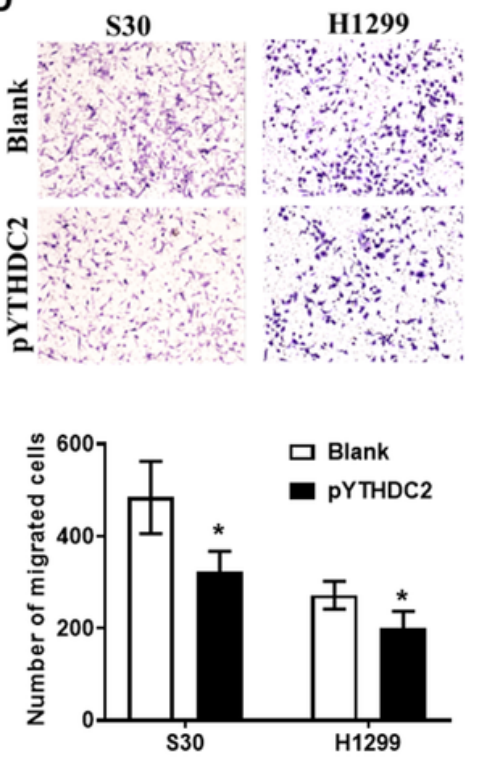

E

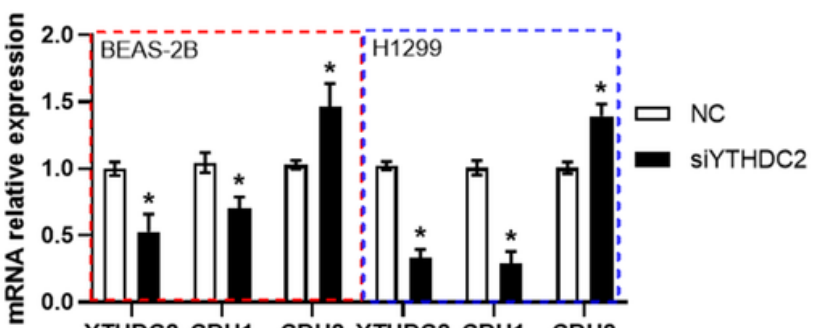

$\mathbf{F}$
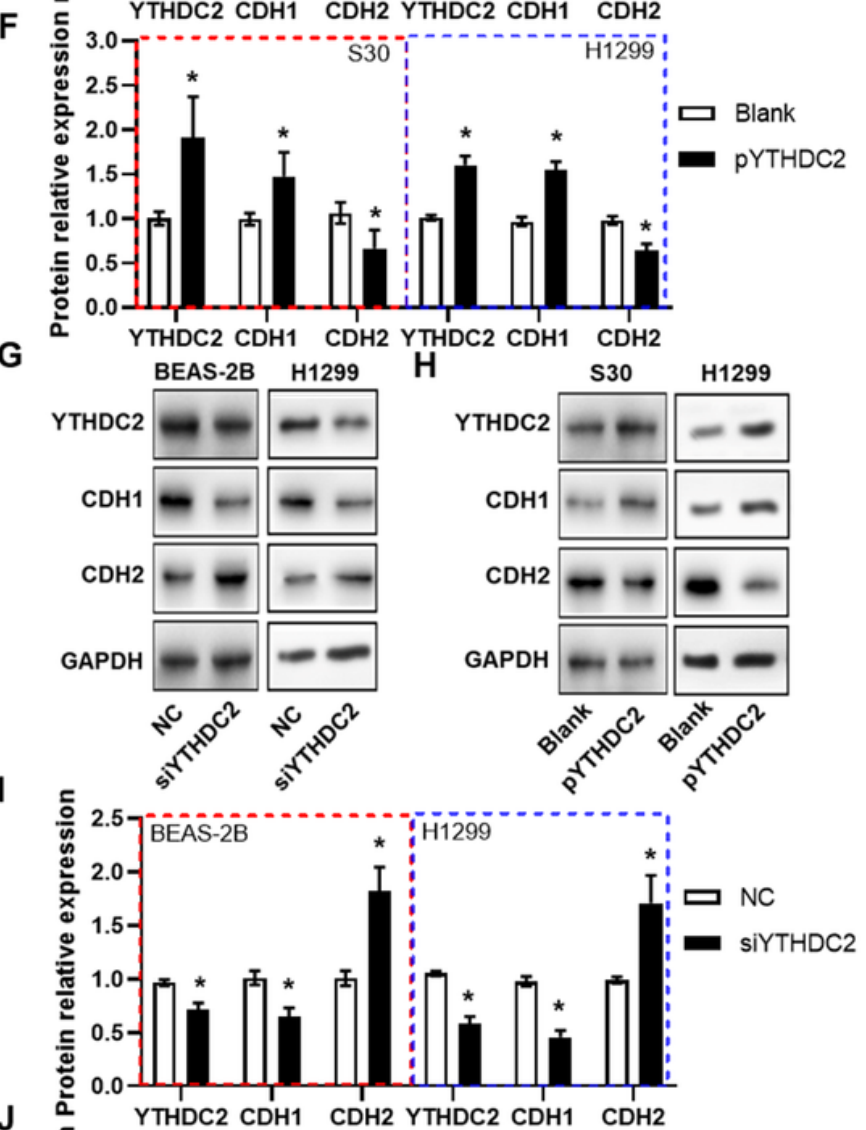

$\mathbf{J}$

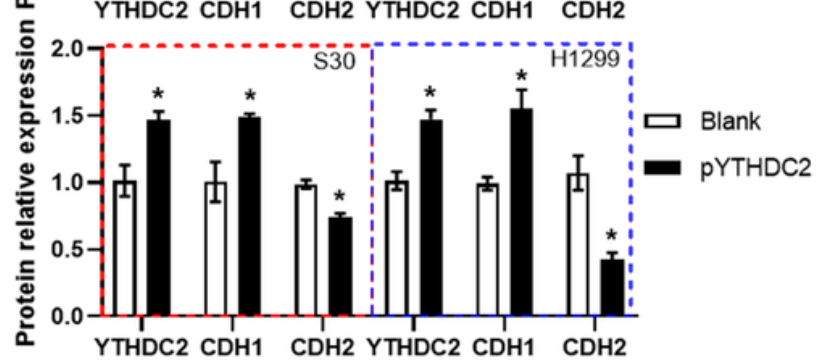

Figure 5

YTHDC2 downregulation promotes lung cancer cell migration. Representative images and quantification of the wound healing assay showing that cell migration is significantly increased at the $24 \mathrm{~h}$ and $48 \mathrm{~h}$ time points after transfected with YTHDC2 siRNA (siYTHDC2) in BEAS-2B and H1299 cells (A), as well as transfected with over-expression vector (pYTHDC2) and blank vector (B). Representative images and quantitation of the transwell migration assay of BEAS-2B and $\mathrm{H} 1299$ cells transfected with siYTHDC2 (C) and pYTHDC2 (D). QPCR analysis of CDH1 and CDH2 in normal BEAS-2B (E) and H1299 (F) cells transfected with siYTHDC2, as well as in $\mathrm{S} 30(\mathrm{G})$ and $\mathrm{H} 1299(\mathrm{H})$ cells transfected with pYTHDC2. Western blot analysis (I) and quantitative results (L) of EMT markers in BEAS-2B and H1299 cells 
transfected with siYTHDC2. Western blot analysis $(\mathrm{J})$ and quantitative results $(\mathrm{M})$ of EMT markers in S30 and H1299 cells transfected with pYTHDC2. EMT: Epithelial-Mesenchymal Transition; NC: negative control. ${ }^{*} p<0.05$ vs. NC (Blank) group; **p < 0.01 vs. NC (Blank) group.

A

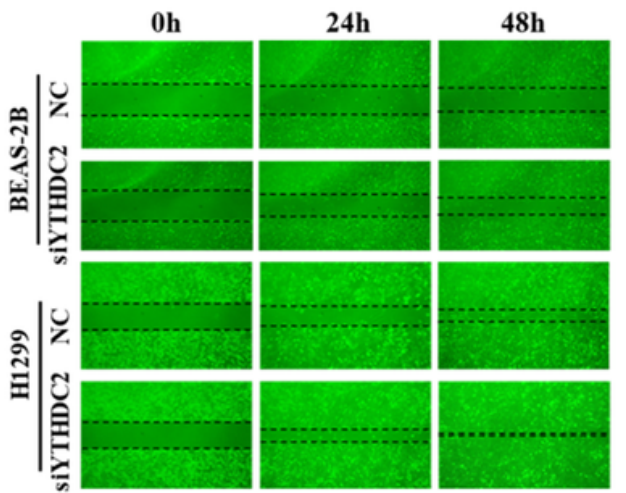

B
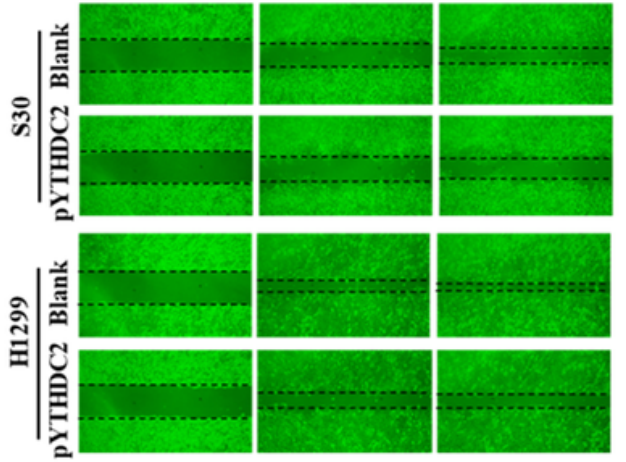

C
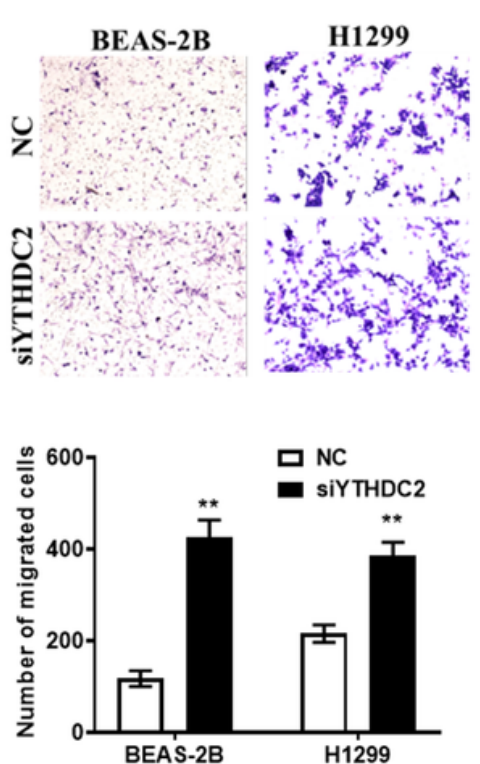
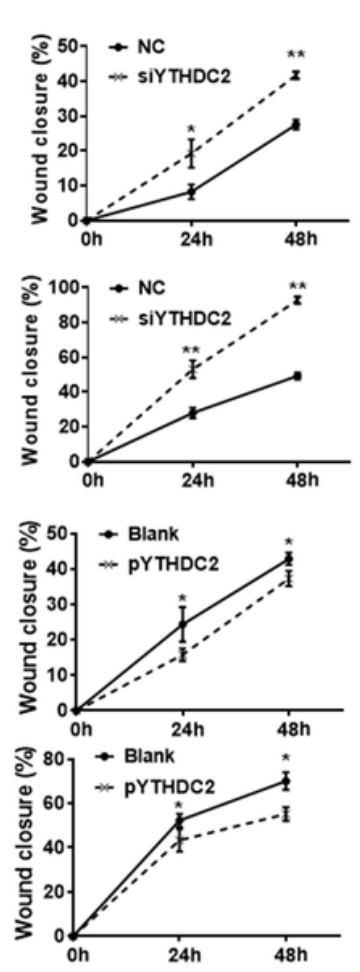

D

S30
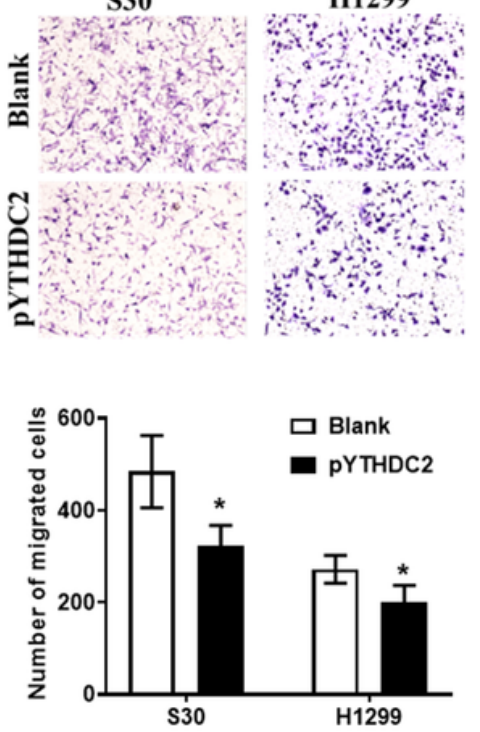

E

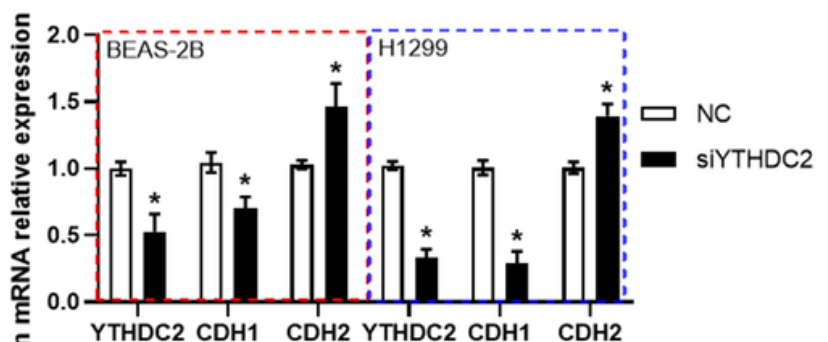

$\mathbf{F}$

G
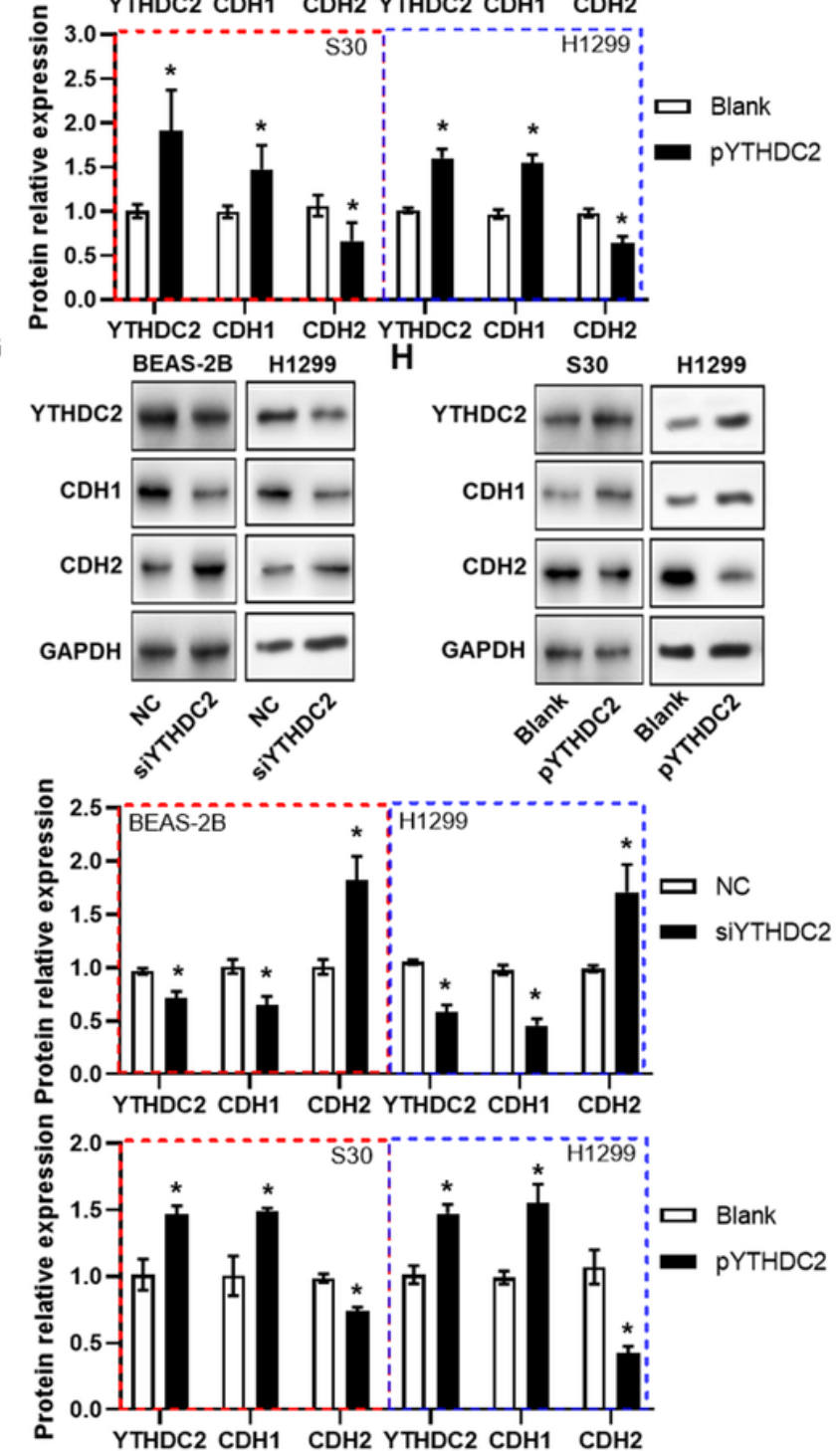

Figure 5

YTHDC2 downregulation promotes lung cancer cell migration. Representative images and quantification of the wound healing assay showing that cell migration is significantly increased at the $24 \mathrm{~h}$ and $48 \mathrm{~h}$ time points after transfected with YTHDC2 siRNA (siYTHDC2) in BEAS-2B and H1299 cells (A), as well as transfected with over-expression vector (pYTHDC2) and blank vector (B). Representative images and quantitation of the transwell migration assay of BEAS-2B and $\mathrm{H} 1299$ cells transfected with siYTHDC2 (C) 
and pYTHDC2 (D). QPCR analysis of CDH1 and CDH2 in normal BEAS-2B (E) and H1299 (F) cells transfected with siYTHDC2, as well as in S30 (G) and H1299 (H) cells transfected with pYTHDC2. Western blot analysis (I) and quantitative results (L) of EMT markers in BEAS-2B and H1299 cells transfected with siYTHDC2. Western blot analysis (J) and quantitative results (M) of EMT markers in S30 and H1299 cells transfected with pYTHDC2. EMT: Epithelial-Mesenchymal Transition; NC: negative control. ${ }^{*} p<0.05$ vs. NC (Blank) group; ${ }^{* *} p<0.01$ vs. NC (Blank) group.

A

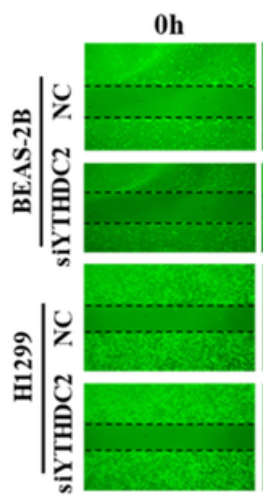

B

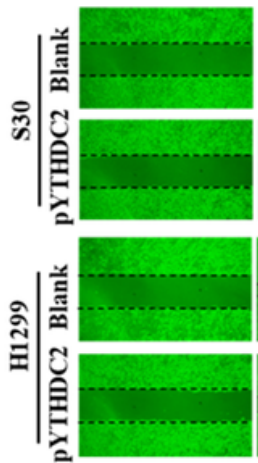

C
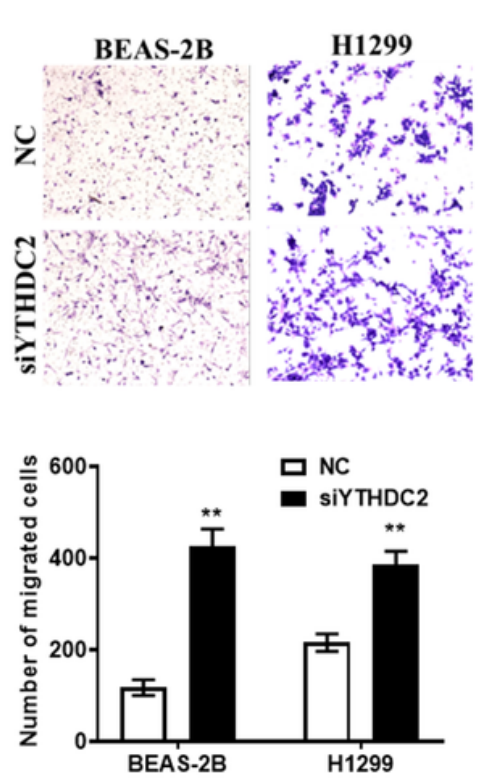
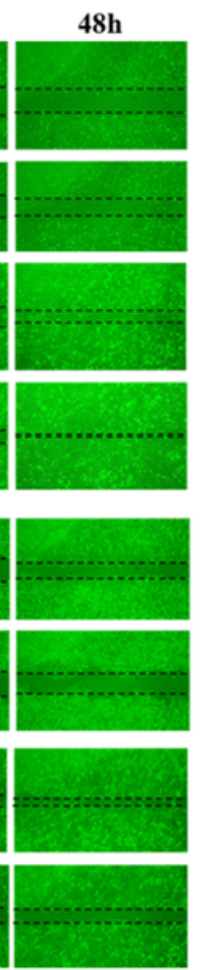

D
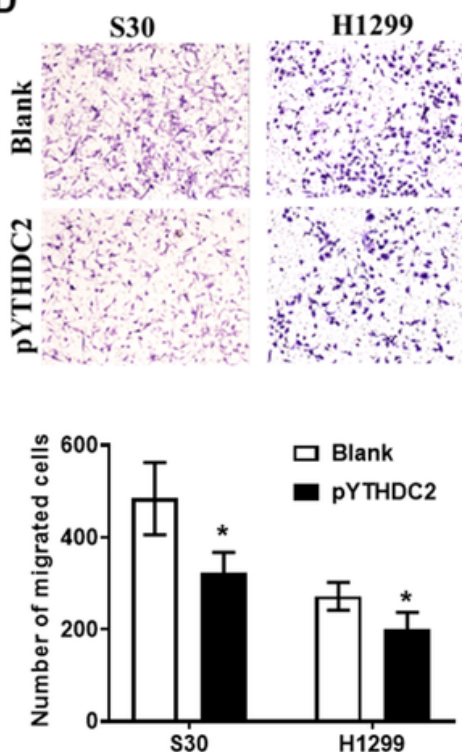

S30

H1299

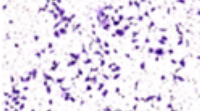

E
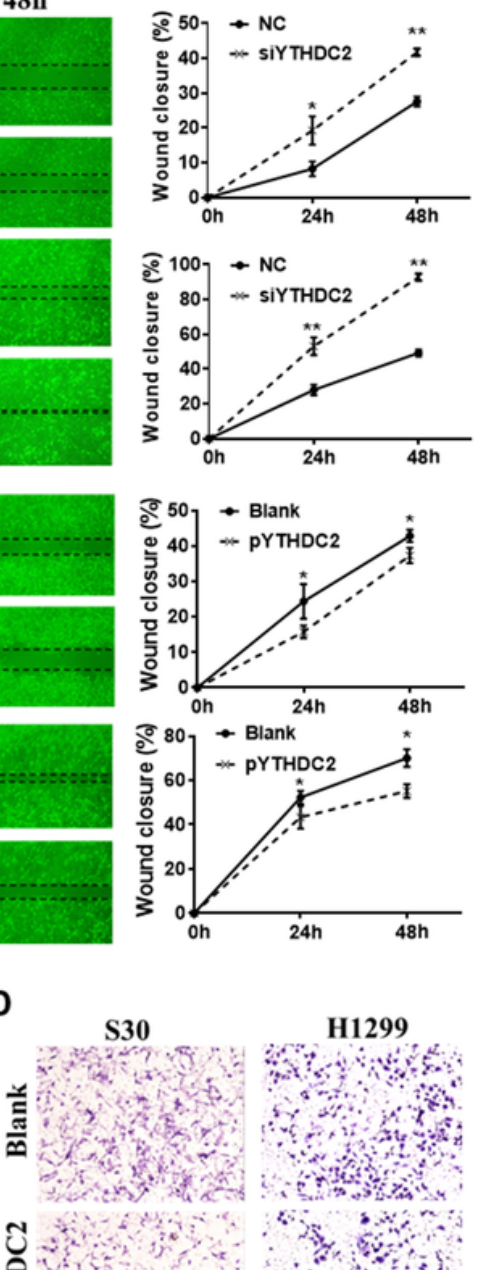

J

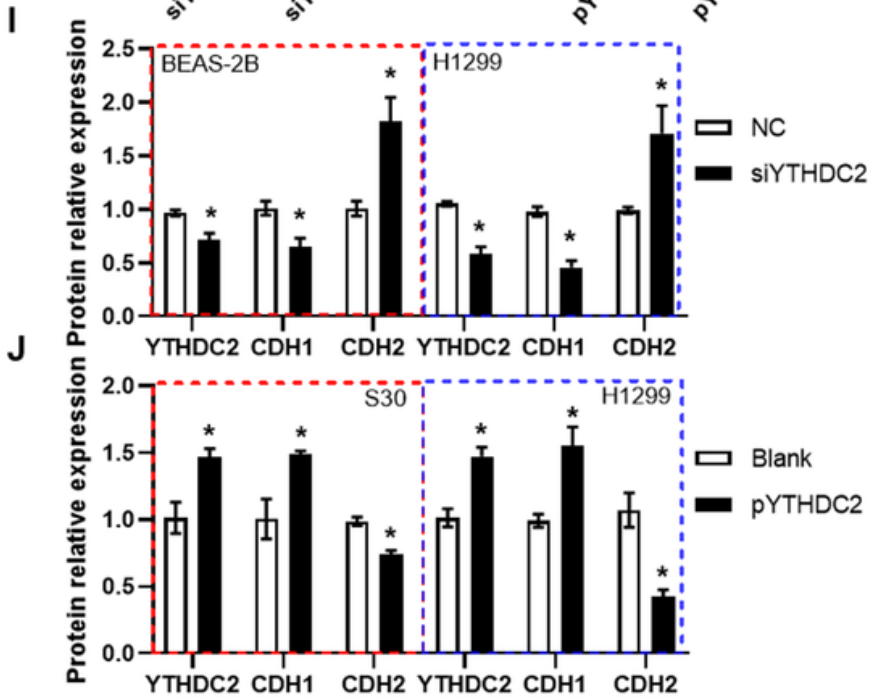

Figure 5

YTHDC2 downregulation promotes lung cancer cell migration. Representative images and quantification of the wound healing assay showing that cell migration is significantly increased at the $24 \mathrm{~h}$ and $48 \mathrm{~h}$ 
time points after transfected with YTHDC2 siRNA (siYTHDC2) in BEAS-2B and H1299 cells (A), as well as transfected with over-expression vector ( $p Y T H D C 2)$ and blank vector (B). Representative images and quantitation of the transwell migration assay of BEAS-2B and $\mathrm{H} 1299$ cells transfected with siYTHDC2 (C) and pYTHDC2 (D). QPCR analysis of CDH1 and CDH2 in normal BEAS-2B (E) and H1299 (F) cells transfected with siYTHDC2, as well as in $\mathrm{S} 30(\mathrm{G})$ and $\mathrm{H} 1299(\mathrm{H})$ cells transfected with pYTHDC2. Western blot analysis (I) and quantitative results (L) of EMT markers in BEAS-2B and H1299 cells transfected with siYTHDC2. Western blot analysis $(\mathrm{J})$ and quantitative results $(\mathrm{M})$ of EMT markers in S30 and H1299 cells transfected with pYTHDC2. EMT: Epithelial-Mesenchymal Transition; NC: negative control. ${ }^{*} p<0.05$ vs. NC (Blank) group; **p $<0.01$ vs. NC (Blank) group.

A
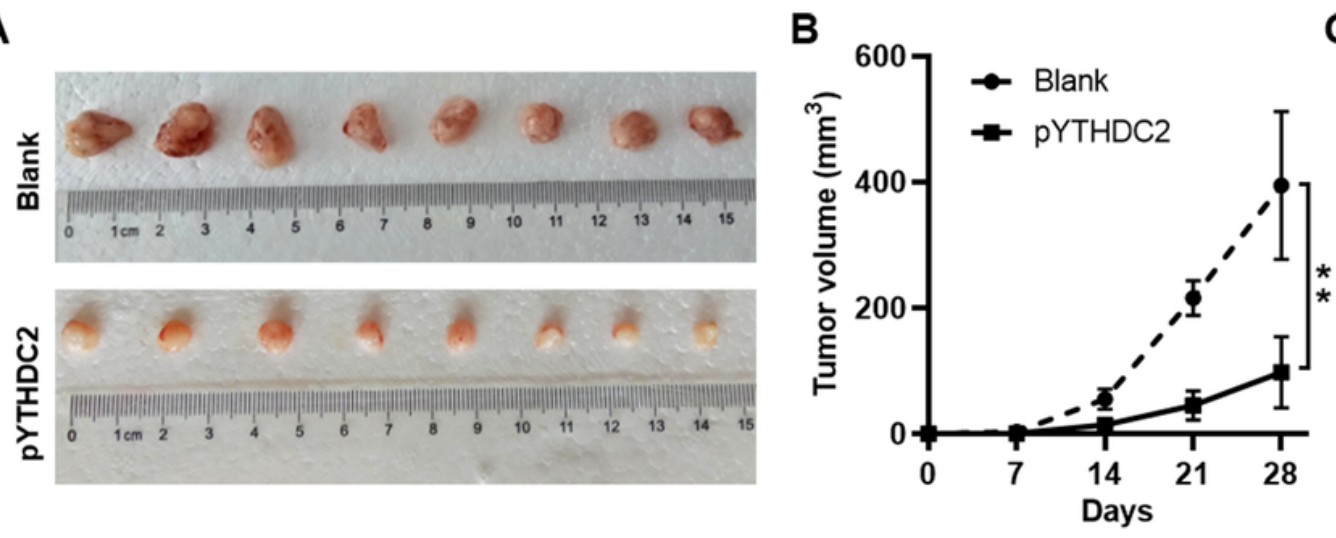

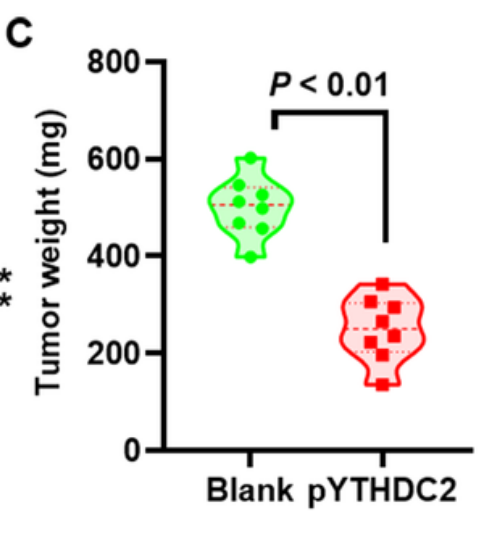

E

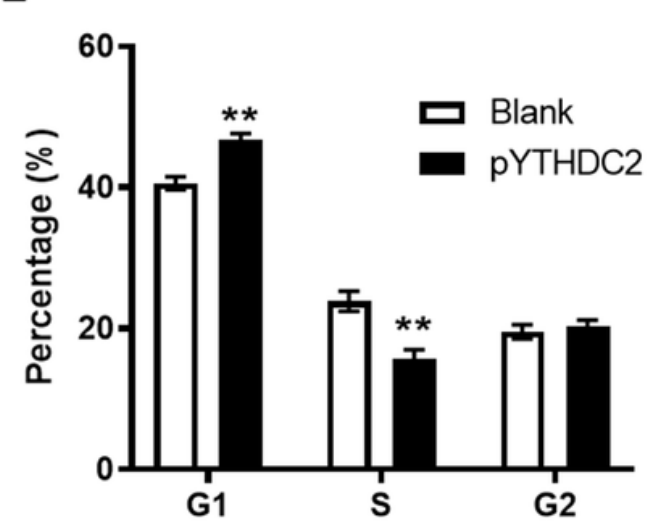

F
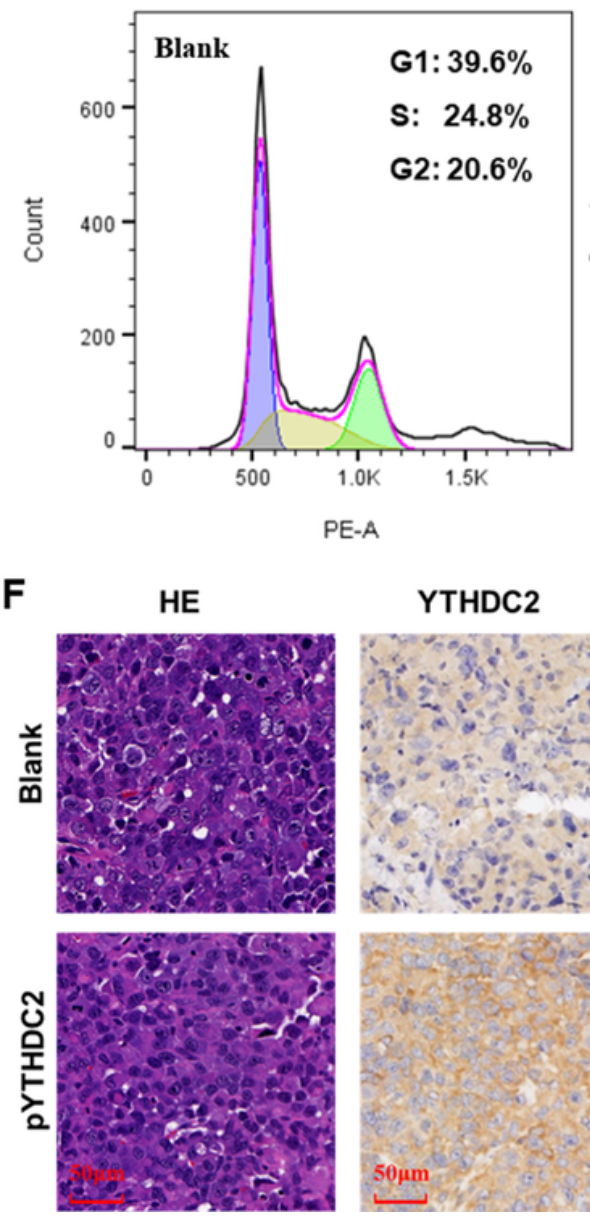

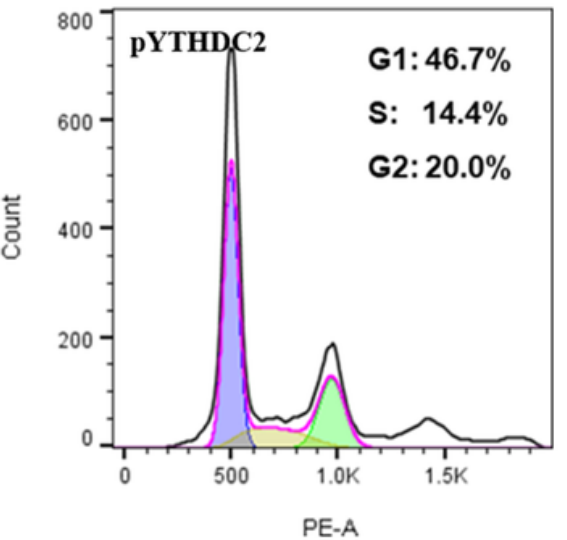

ki67
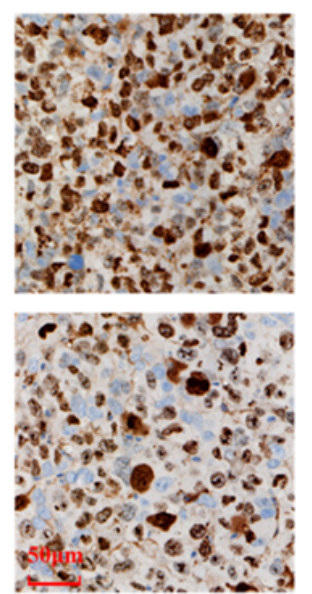

Cyclin D1
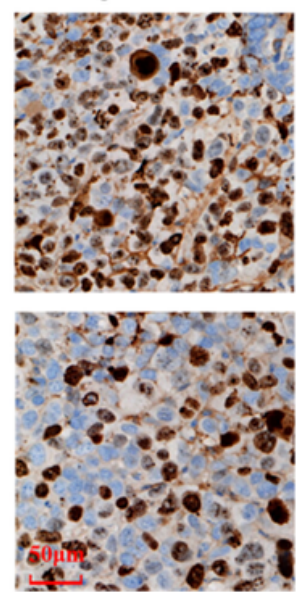

CDH1
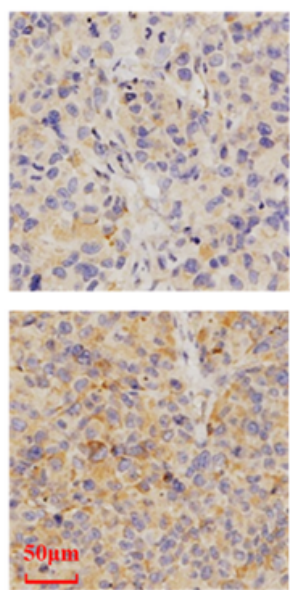

$\mathrm{CDH} 2$

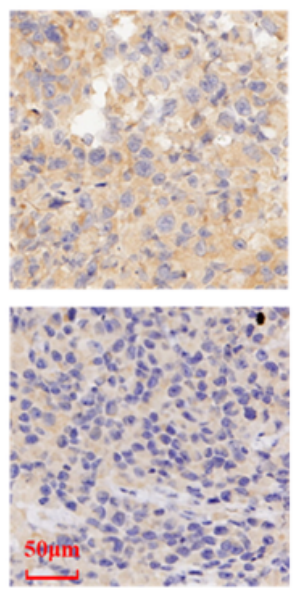

Figure 6 
YTHDC2 overexpression suppresses H1299 cells growth in vivo. (A) Images of the xenograft tumors formed in nude mice injected with YTHDC2 overexpression and control cells. The volume (B) and weight (C) of the xenograft tumors isolated from nude mice. Representative images (D) and quantification result (E) of cell cycle of single cell suspension yielded from xenograft tumors. (F) Representative images of H\&E staining, and the immunohistochemical (IHC) staining of YTHDC2, Ki-67, Cyclin D1, CDH1 and CDH2 in the xenograft tumors derived from nude mice. $\mathrm{CDH} 1$, E-cadherin; $\mathrm{CDH} 2, \mathrm{~N}$-cadherin. Scale bar $=50 \mu \mathrm{m}$; $\star * p<0.01$ vs. NC (Blank) group.

A

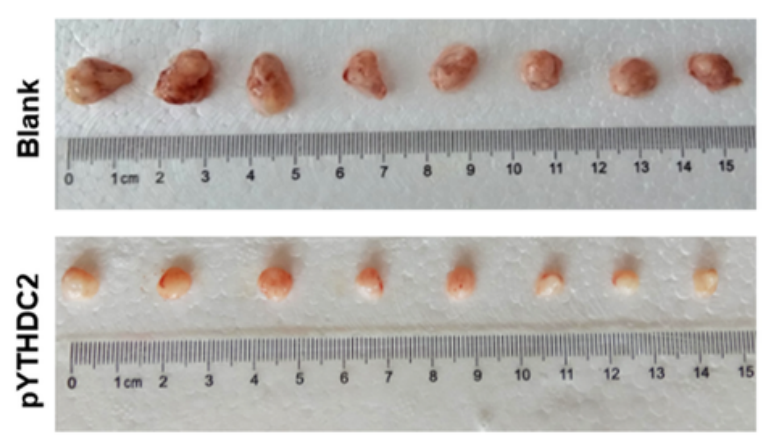

D

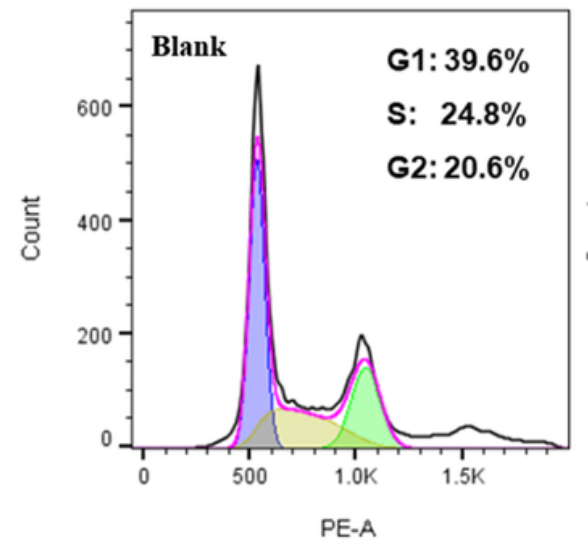

$\mathbf{F}$
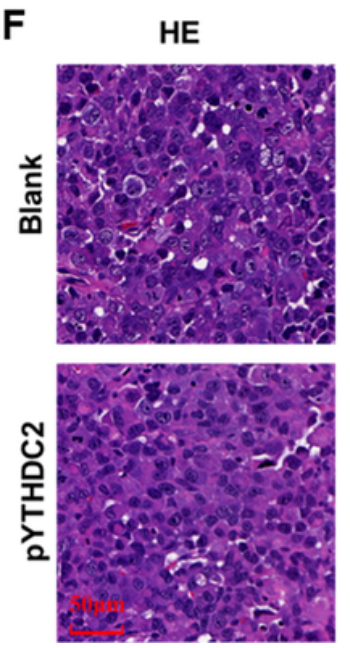
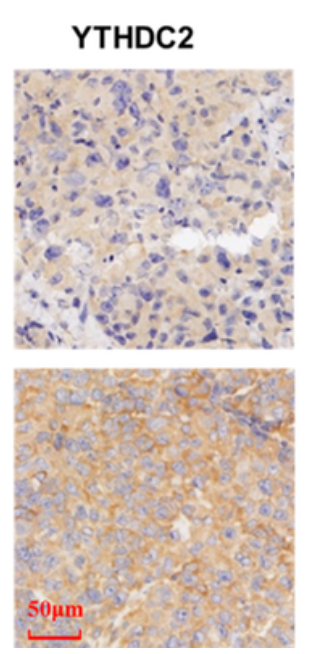

B

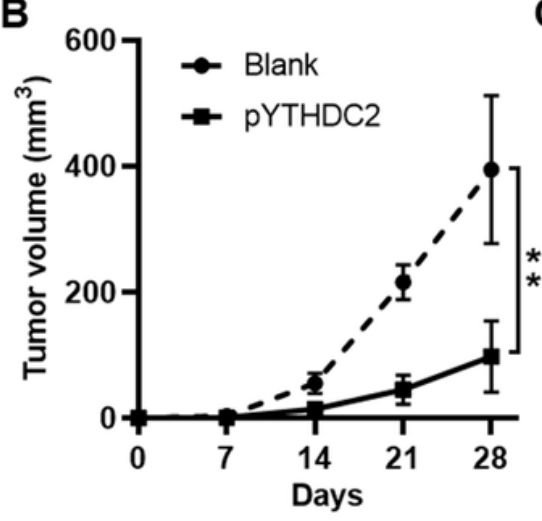

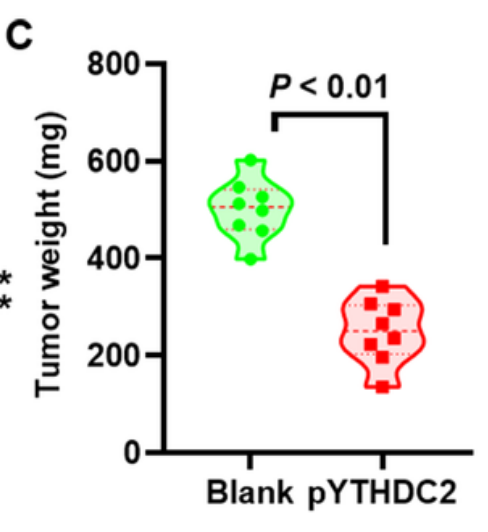

E

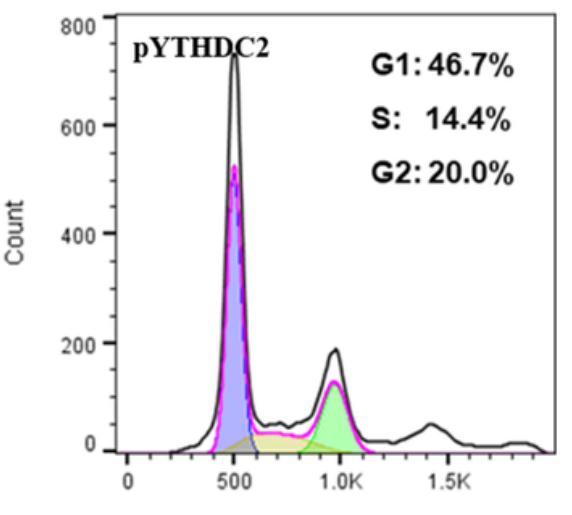

PE-A
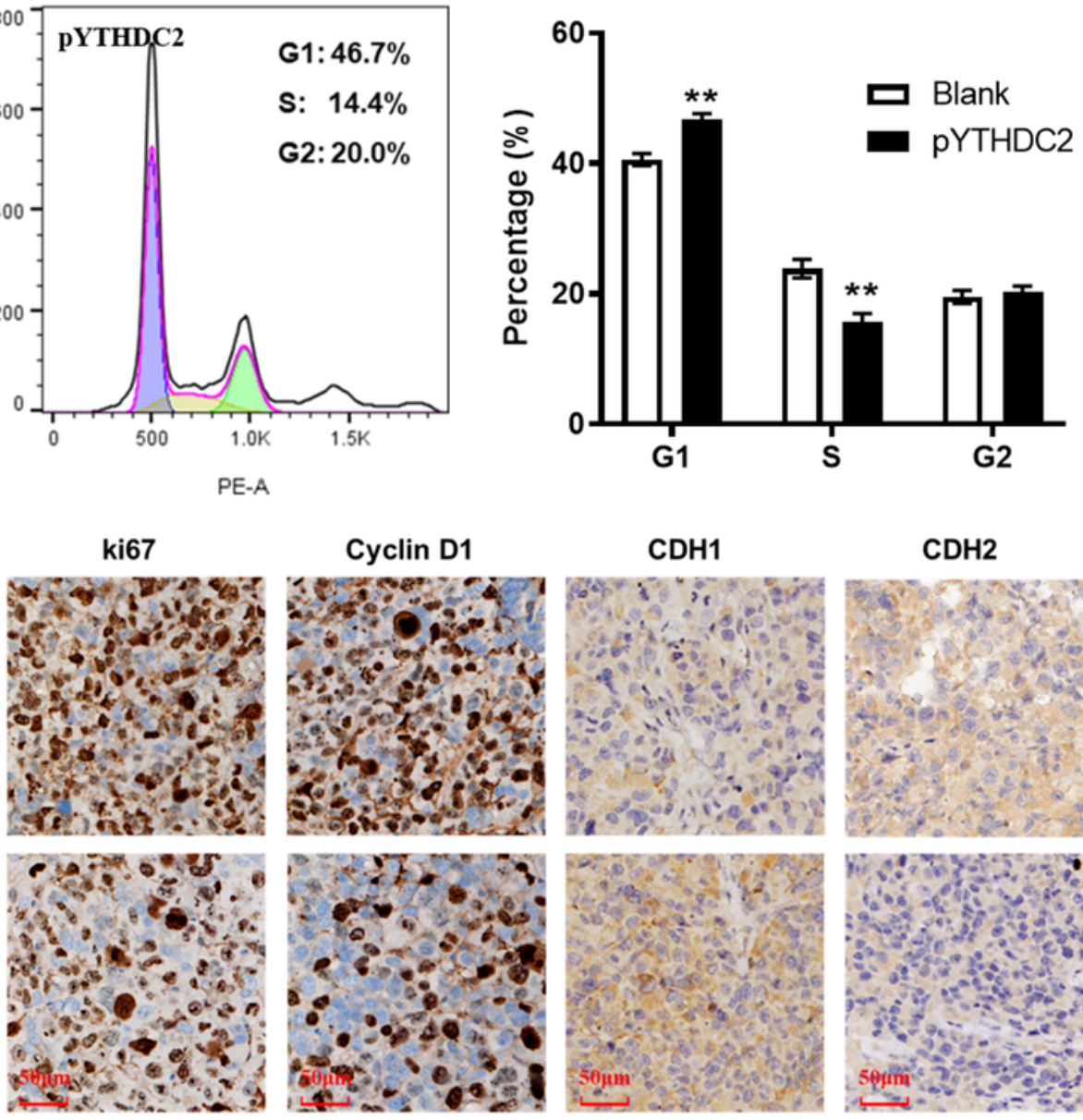

CDH1
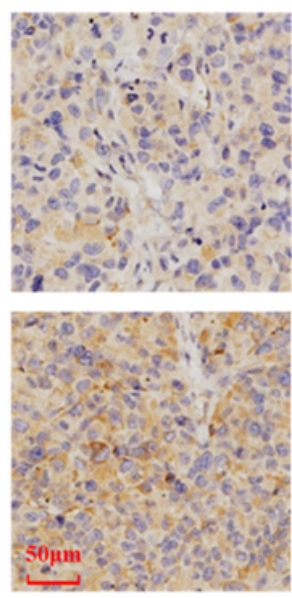

CDH2
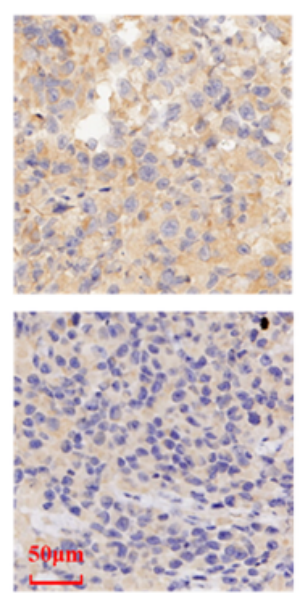

Figure 6 
YTHDC2 overexpression suppresses H1299 cells growth in vivo. (A) Images of the xenograft tumors formed in nude mice injected with YTHDC2 overexpression and control cells. The volume (B) and weight (C) of the xenograft tumors isolated from nude mice. Representative images (D) and quantification result (E) of cell cycle of single cell suspension yielded from xenograft tumors. (F) Representative images of H\&E staining, and the immunohistochemical (IHC) staining of YTHDC2, Ki-67, Cyclin D1, CDH1 and CDH2 in the xenograft tumors derived from nude mice. $\mathrm{CDH} 1$, E-cadherin; $\mathrm{CDH} 2, \mathrm{~N}$-cadherin. Scale bar $=50 \mu \mathrm{m}$; $\star * p<0.01$ vs. NC (Blank) group.

A
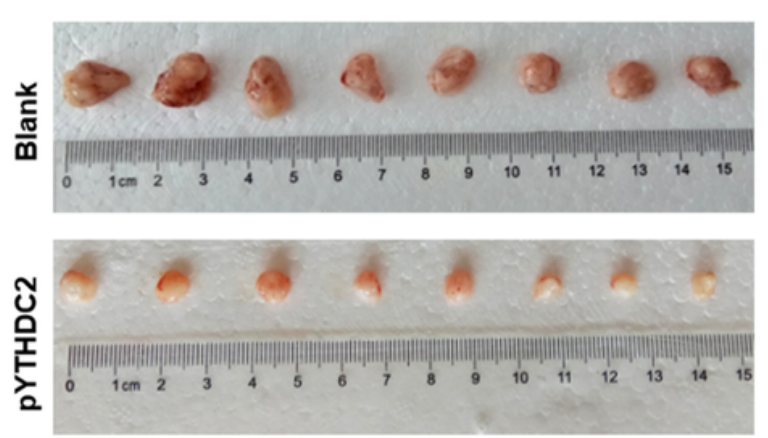

D

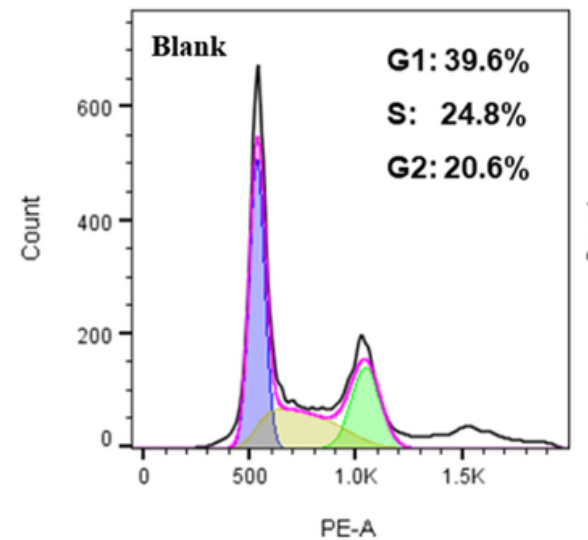

$\mathbf{F}$
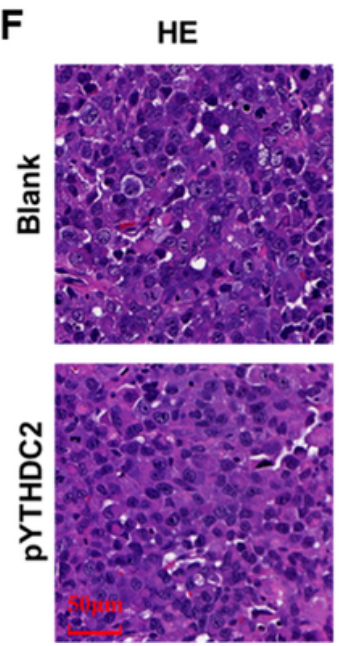
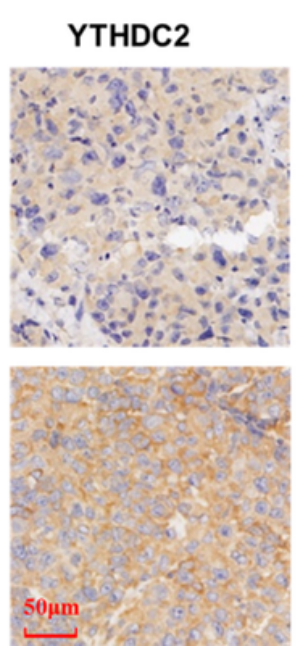

B

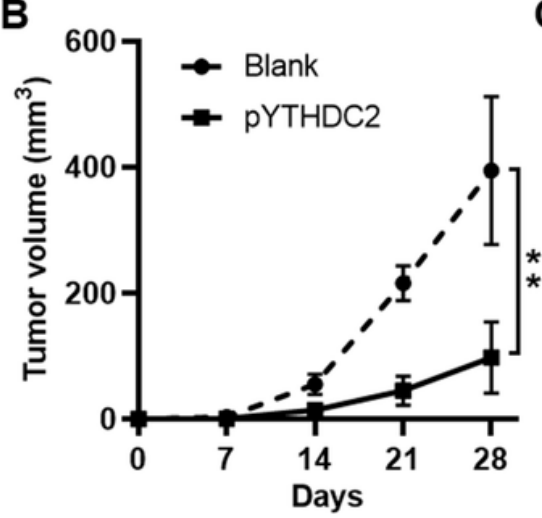

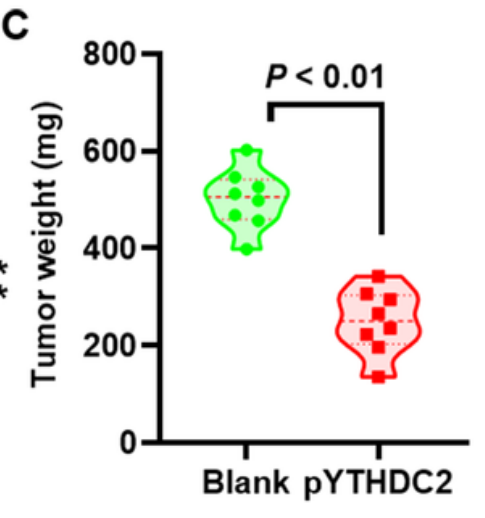

E

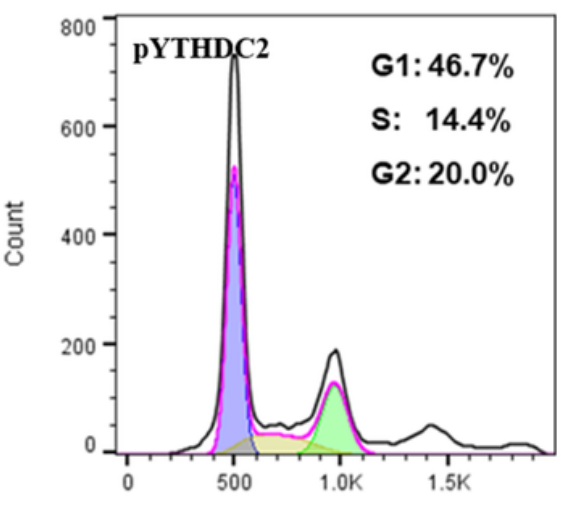

PE-A
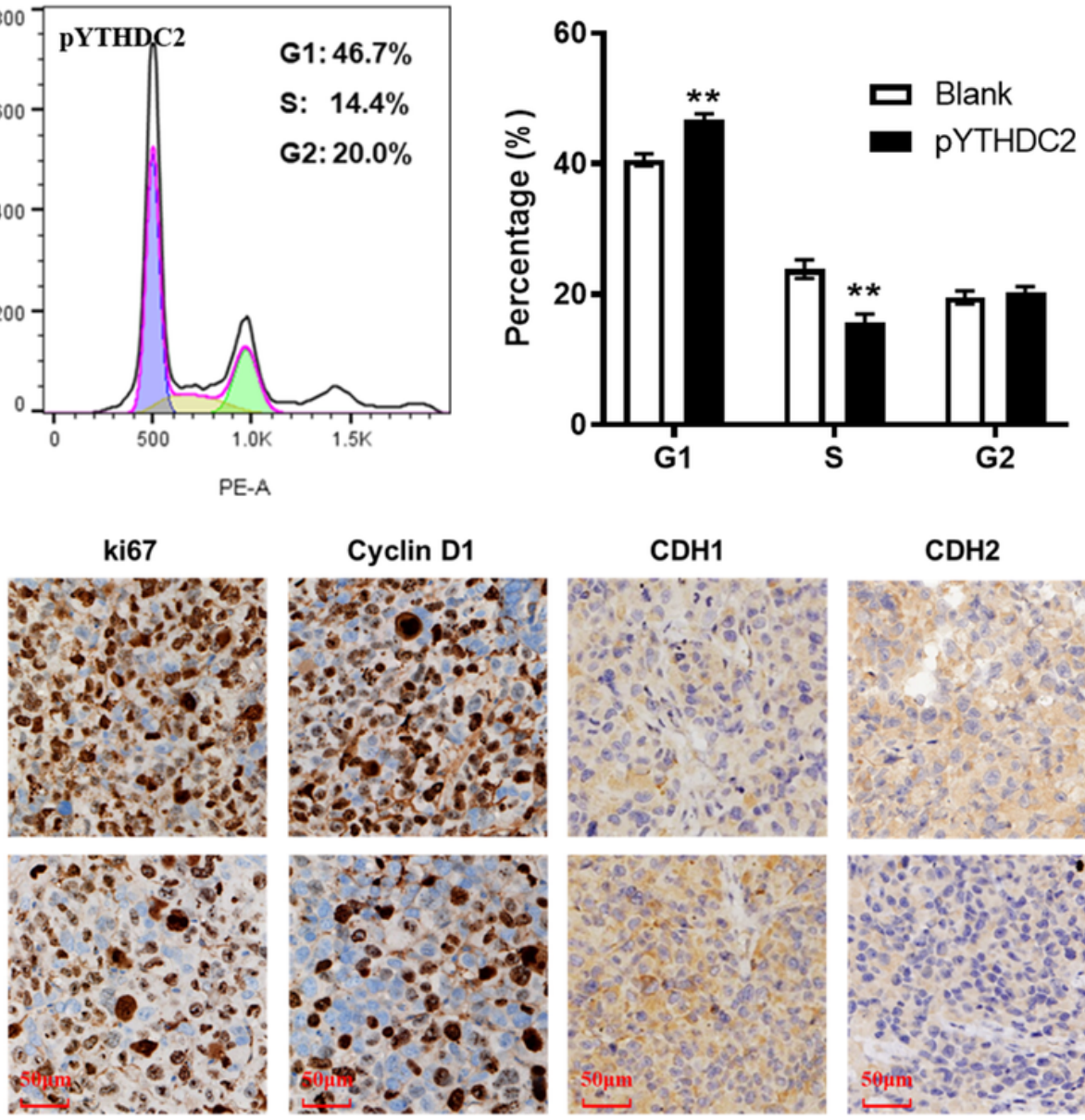

CDH1
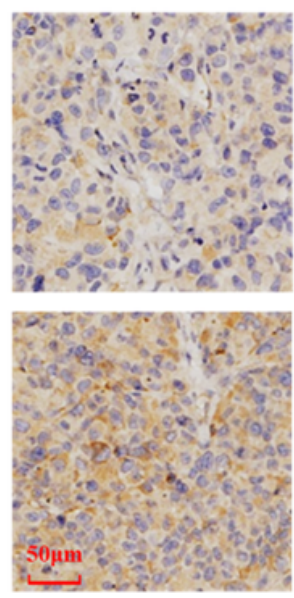

CDH2
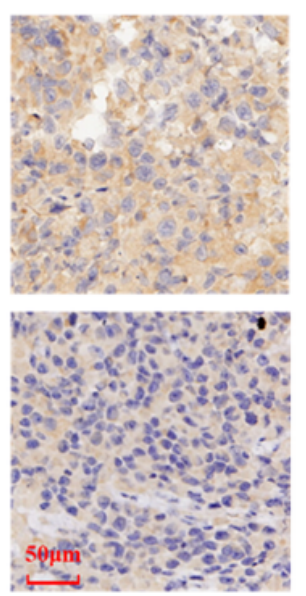

Figure 6 
YTHDC2 overexpression suppresses H1299 cells growth in vivo. (A) Images of the xenograft tumors formed in nude mice injected with YTHDC2 overexpression and control cells. The volume (B) and weight (C) of the xenograft tumors isolated from nude mice. Representative images (D) and quantification result (E) of cell cycle of single cell suspension yielded from xenograft tumors. (F) Representative images of H\&E staining, and the immunohistochemical (IHC) staining of YTHDC2, Ki-67, Cyclin D1, CDH1 and CDH2 in the xenograft tumors derived from nude mice. $\mathrm{CDH} 1$, E-cadherin; $\mathrm{CDH} 2, \mathrm{~N}$-cadherin. Scale bar = 50 $\mu$ m; ${ }^{* *} \mathrm{p}<0.01$ vs. NC (Blank) group.
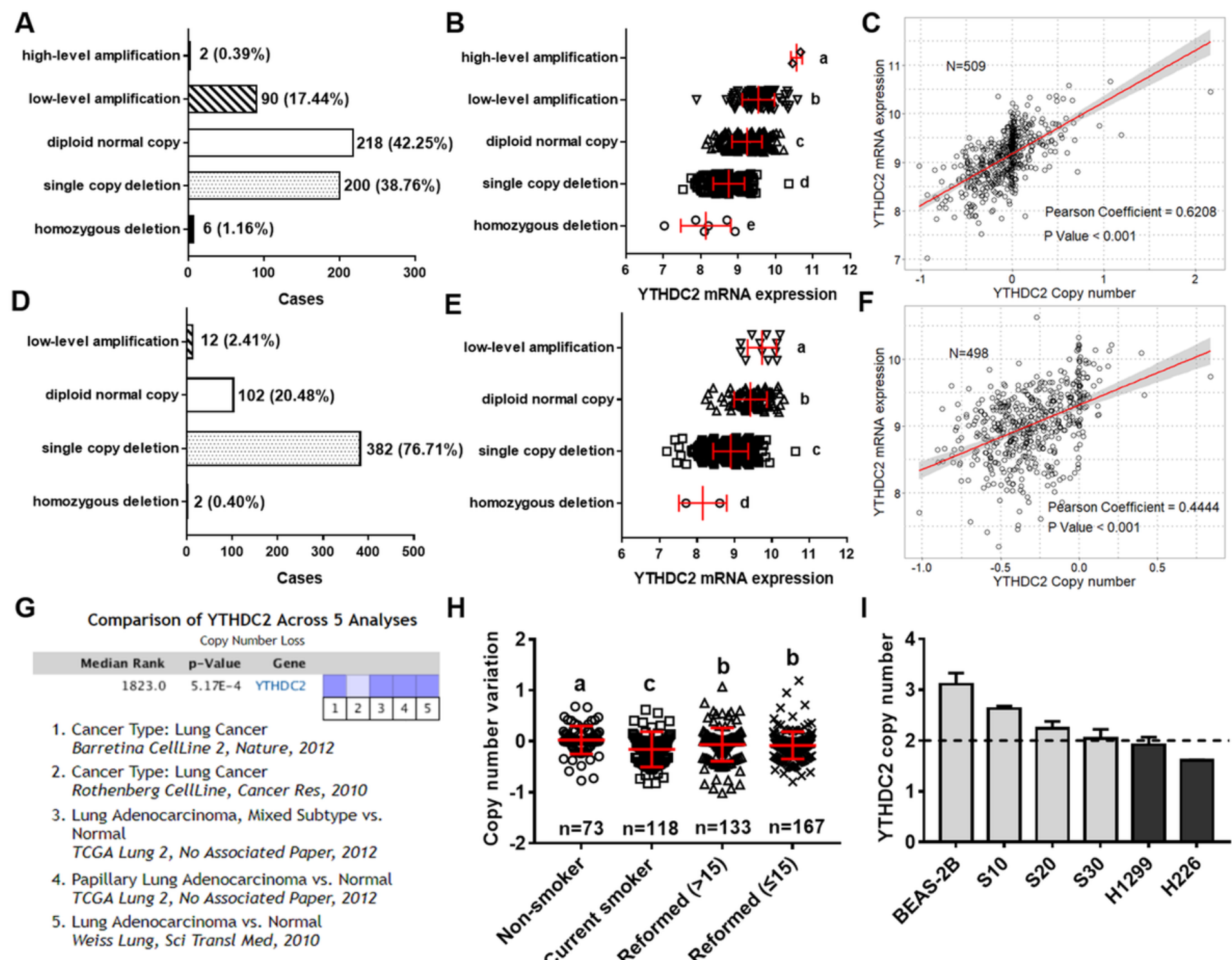

E YTHDC2 mRNA expression $F$

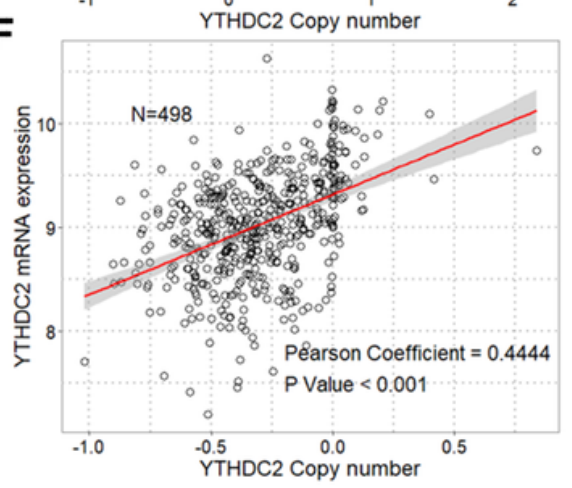

H

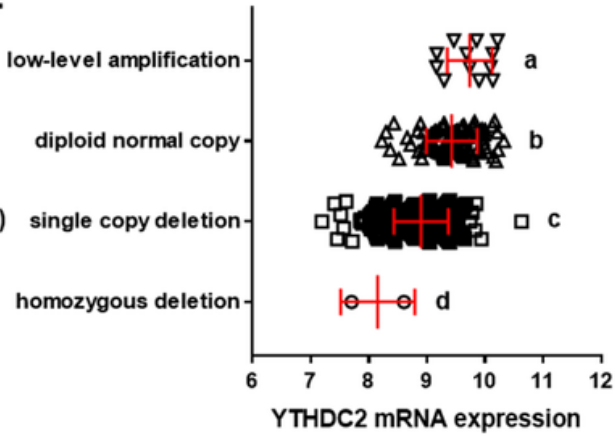

I
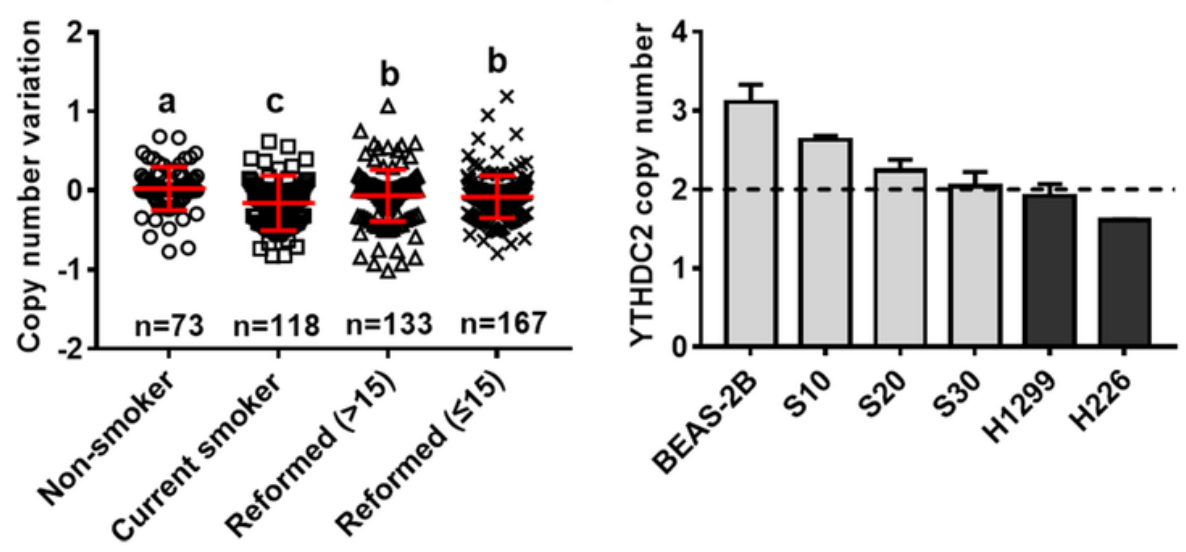

\section{Figure 7}

YTHDC2 mRNA expression was regulated by gene amplification. Distribution of LUAD (A) and LUSC (D) patients with different YTHDC2 amplification status. YTHDC2 mRNA expression in LUAD (B) and LUSC (E) tissues with different YTHDC2 amplification status. Different letters ( $a, b, c$, and d) represent statistically significant group differences. Pearson correlation analysis showed a significant positive correlation between YTHDC2 mRNA expression and copy numbers in LUAD (C) and LUSC (F). Line represents linear regression of data (LUAD: $y=1.065 x+9.177, R 2=0.385$; LUSC: $y=0.965 x+9.318, R 2=$ 
0.198). (G) The oncomine datasets for respective YTHDC2 copy numbers in lung cancer were retried under the parameters with a threshold $p$ value of 0.001 and a minimum 2 -fold change. The data in the graphic show significant downregulation (blue column) of the YTHDC2 copy numbers in lung cancer versus normal tissue. The intensity of blue represents the respective levels of YTHDC2 copy number. $(H)$ Copy number variation in LUAD samples with different smoking histories. Different letters $(a, b, c$, and $d)$ represent statistically significant group differences. (I) The copy number variation of YTHDC2 in BEAS-2B cells and cigarette smoke-exposed cells (grey block), as well as two lung cancer cell lines (black block). The dotted line (copy number $=2$ ) represents the copy number of the reference gene RNase P.
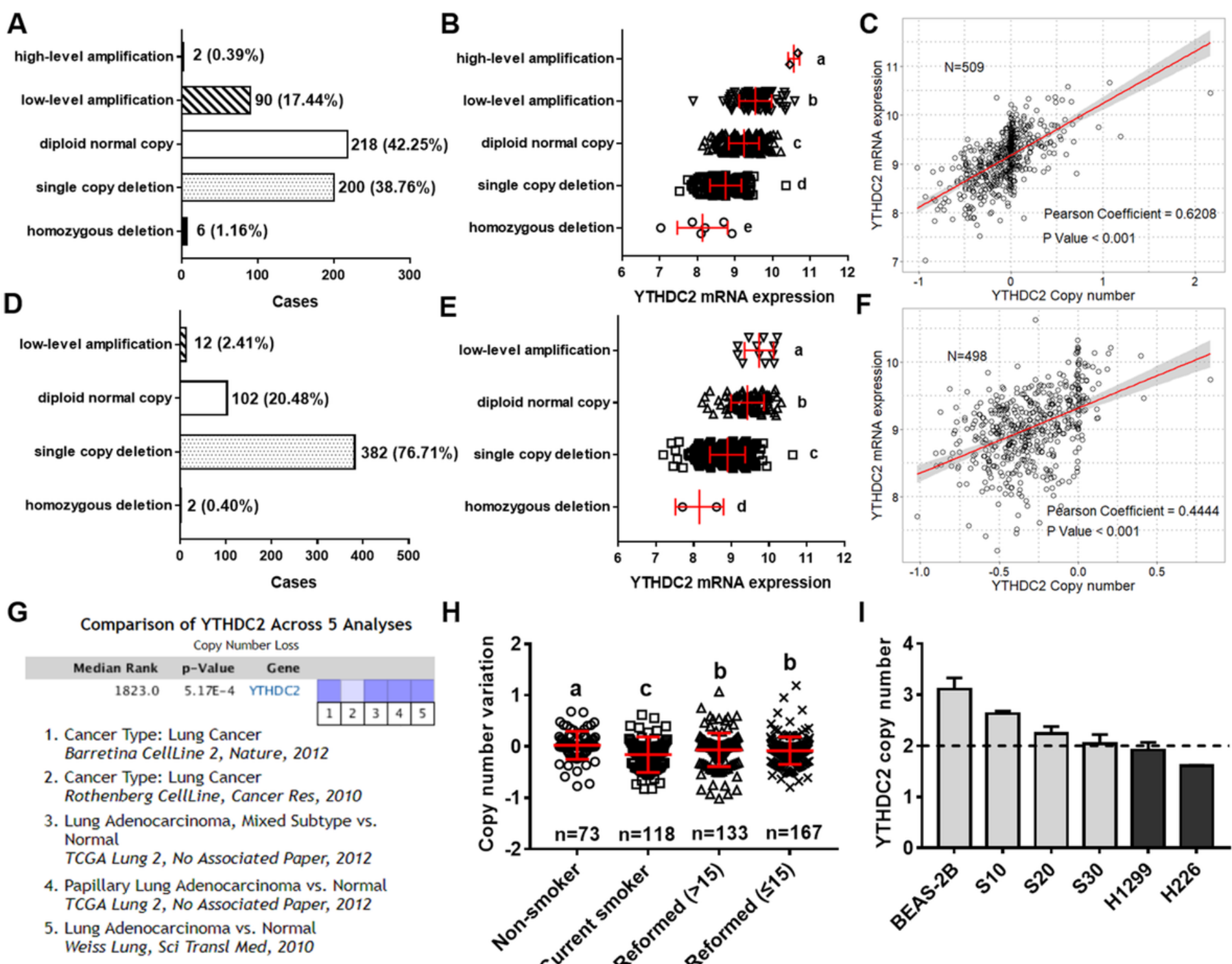

E
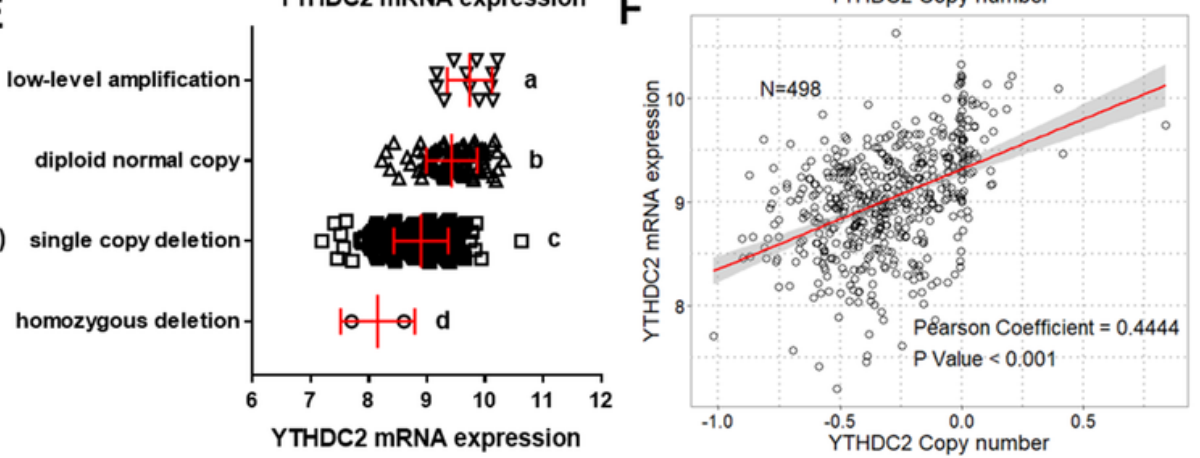

H

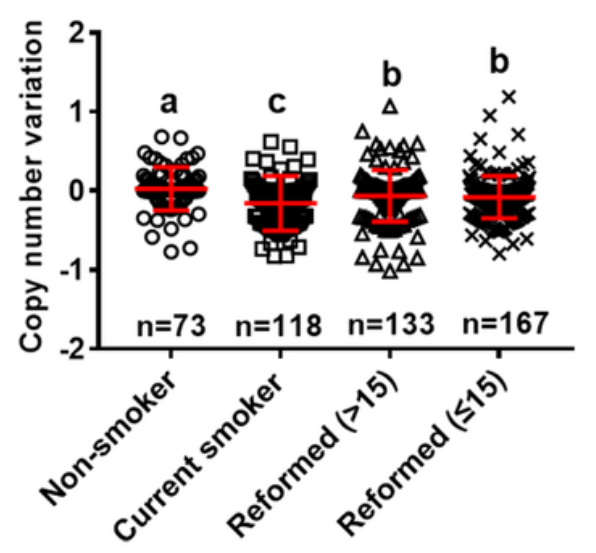

I

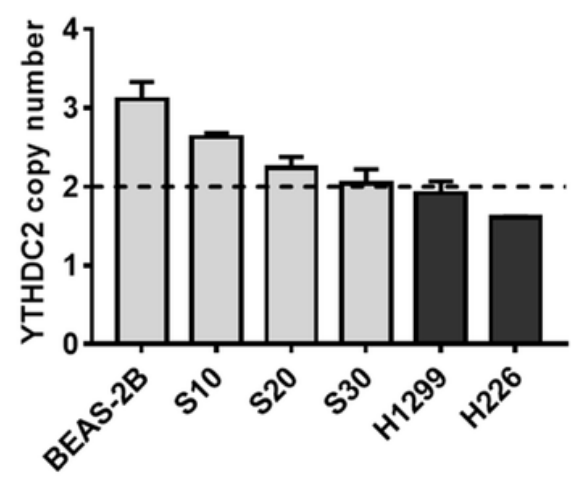

\section{Figure 7}

YTHDC2 mRNA expression was regulated by gene amplification. Distribution of LUAD (A) and LUSC (D) patients with different YTHDC2 amplification status. YTHDC2 mRNA expression in LUAD (B) and LUSC (E) tissues with different YTHDC2 amplification status. Different letters (a, b, c, and d) represent statistically significant group differences. Pearson correlation analysis showed a significant positive correlation between YTHDC2 mRNA expression and copy numbers in LUAD (C) and LUSC (F). Line 
represents linear regression of data (LUAD: $y=1.065 x+9.177, R 2=0.385$; LUSC: $y=0.965 x+9.318, R 2=$ 0.198). (G) The oncomine datasets for respective YTHDC2 copy numbers in lung cancer were retried under the parameters with a threshold $p$ value of 0.001 and a minimum 2 -fold change. The data in the graphic show significant downregulation (blue column) of the YTHDC2 copy numbers in lung cancer versus normal tissue. The intensity of blue represents the respective levels of YTHDC2 copy number. $(H)$ Copy number variation in LUAD samples with different smoking histories. Different letters $(a, b, c$, and d) represent statistically significant group differences. (I) The copy number variation of YTHDC2 in BEAS-2B cells and cigarette smoke-exposed cells (grey block), as well as two lung cancer cell lines (black block). The dotted line (copy number $=2$ ) represents the copy number of the reference gene RNase P.
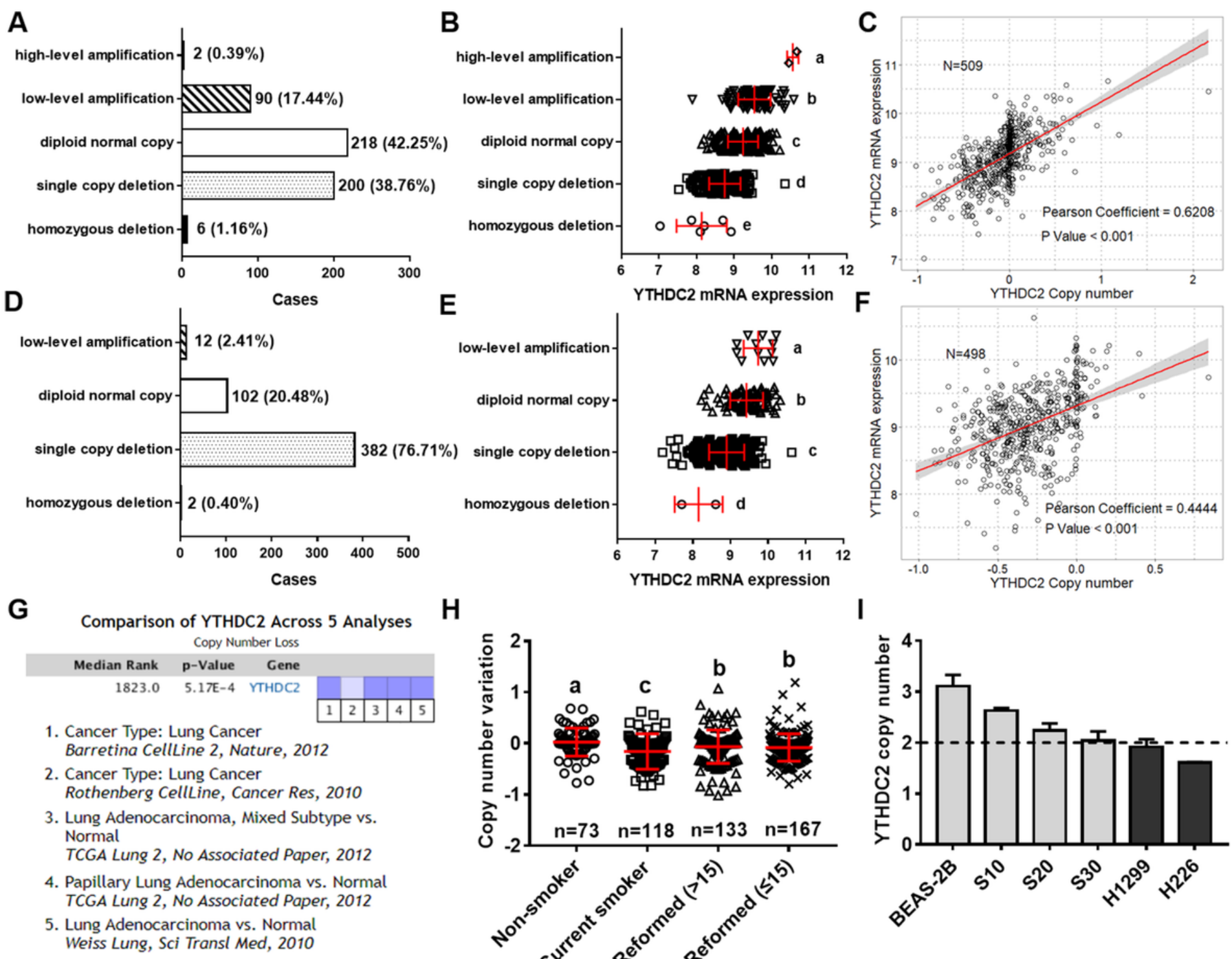

E
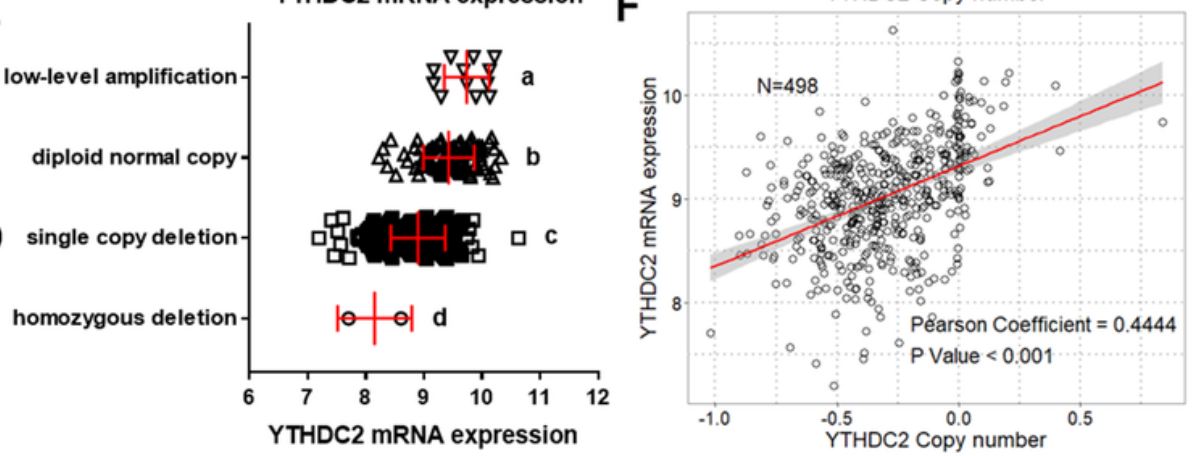

H

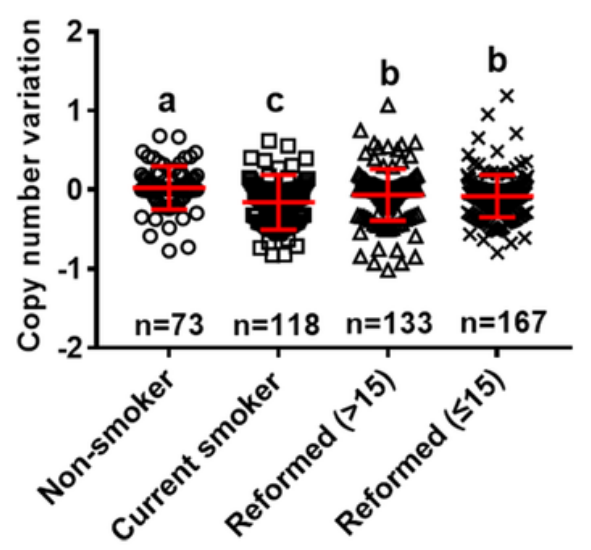

I

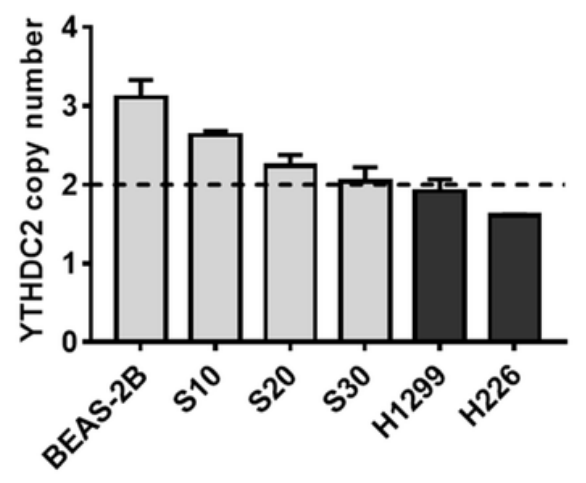

\section{Figure 7}

YTHDC2 mRNA expression was regulated by gene amplification. Distribution of LUAD (A) and LUSC (D) patients with different YTHDC2 amplification status. YTHDC2 mRNA expression in LUAD (B) and LUSC (E) tissues with different YTHDC2 amplification status. Different letters (a, b, c, and d) represent statistically significant group differences. Pearson correlation analysis showed a significant positive 
correlation between YTHDC2 mRNA expression and copy numbers in LUAD (C) and LUSC (F). Line represents linear regression of data (LUAD: $y=1.065 x+9.177, R 2=0.385$; LUSC: $y=0.965 x+9.318, R 2=$ 0.198). (G) The oncomine datasets for respective YTHDC2 copy numbers in lung cancer were retried under the parameters with a threshold $p$ value of 0.001 and a minimum 2-fold change. The data in the graphic show significant downregulation (blue column) of the YTHDC2 copy numbers in lung cancer versus normal tissue. The intensity of blue represents the respective levels of YTHDC2 copy number. $(H)$ Copy number variation in LUAD samples with different smoking histories. Different letters (a, b, c, and d) represent statistically significant group differences. (I) The copy number variation of YTHDC2 in BEAS-2B cells and cigarette smoke-exposed cells (grey block), as well as two lung cancer cell lines (black block). The dotted line (copy number $=2$ ) represents the copy number of the reference gene RNase P.

\section{Supplementary Files}

This is a list of supplementary files associated with this preprint. Click to download.

- Additionalfile1.docx

- Additionalfile1.docx

- Additionalfile1.docx

- OnlineGraphicalAbstract.png

- OnlineGraphicalAbstract.png

- OnlineGraphicalAbstract.png 